\title{
The Gambia: 2008 Article IV Consultation and Third Review Under the Three-Year Arrangement Under the Poverty Reduction and Growth Facility, and Request for Waiver of Performance Criteria-Staff Report; Staff Supplement; Public Information Notice and Press Release on the Executive Board Discussion; and Statement by the Executive Director for The Gambia
}

Under Article IV of the IMF's Articles of Agreement, the IMF holds bilateral discussions with members, usually every year. In the context of a combined discussion of the 2008 Article IV consultation with The Gambia and the third review of the three-year arrangement under the Poverty Reduction and Growth Facility and a request for a waiver of performance criteria, the following documents have been released and are included in this package:

- $\quad$ The staff report for the combined 2008 Article IV Consultation and Third Review Under the Three-Year Arrangement Under the Poverty Reduction and Growth Facility, and Request for Waiver of Performance Criteria, prepared by a staff team of the IMF, following discussions that ended on May 25, 2008 with officials of The Gambia on economic developments and policies. Based on information available at end-July 2008, the staff report was completed on August 20, 2008. The views expressed in the staff report are those of the staff team and do not necessarily reflect the views of the Executive Board of the IMF.

- $\quad$ A staff supplement on the joint IMF/World Bank debt sustainability analysis update.

- $\quad$ A public information notice (PIN) and Press Release, summarizing the views of the Executive Board as expressed during its September 8, 2008 discussion of the staff report that concluded the Article IV consultation and completed the review and request, respectively.

- $\quad$ A statement by the Executive Director for The Gambia

The documents listed below have been or will be separately released.

Letter of Intent sent to the IMF by the authorities of The Gambia*

Memorandum of Economic and Financial Policies by the authorities of The Gambia*

Technical Memorandum of Understanding*

Selected Issues and Statistical Appendix

*Also included in the Staff Report

The policy of publication of staff reports and other documents allows for the deletion of market-sensitive information.

Copies of this report are available to the public from

International Monetary Fund • Publication Services

$70019^{\text {th }}$ Street, N.W. • Washington, D.C. 20431

Telephone: (202) 623-7430 • Telefax: (202) 623-7201

E-mail: publications@imf.org Internet: http://www.imf.org

Price: $\$ 18.00$ a copy

\section{International Monetary Fund Washington, D.C.}





\title{
INTERNATIONAL MONETARY FUND
}

THE GAMBIA

\section{Staff Report for the 2008 Article IV Consultation, Third Review Under the Three-Year Arrangement Under the Poverty Reduction and Growth Facility, and Request for Waiver of Performance Criteria}

\author{
Prepared by the African Department \\ (In consultation with other departments)
}

Approved by Hugh Bredenkamp and Mark Plant

August 20, 2008

- A mission visited Banjul May 11-25, 2008 to conduct discussions for the 2008 Article IV consultation and third review under the Poverty Reduction and Growth Facility (PRGF) arrangement. The mission team comprised Messrs. Tsikata (head), Dwight, Sriram (all AFR), Ms. Amornvivat (PDR), and Mr. Segura (Resident Representative). Ms. Lephoto (OED) participated in the discussions.

- The mission met with Secretary of State for Finance and Economic Affairs Mousa Gibril Bala-Gaye, Governor of the Central Bank of The Gambia (CBG) Momodou Bamba Saho, other senior officials of the government and the CBG, the Speaker and other members of the national assembly, and representatives of commercial banks, the business community, civil society, and The Gambia's development partners.

- The Gambia's exchange rate regime is a managed float. It accepted the obligations under Article VIII, Sections 2(a), 3, and 4, of the Fund's Articles of Agreement on January 21, 1993. The authorities have notified the Fund that for security reasons, and in line with UN Security Council resolutions, they have imposed some restrictions on the making of payments and transfers for current international transactions.

- The Executive Board approved a three-year PRGF arrangement for The Gambia in February 2007 in the amount of SDR 14 million (45 percent of quota). The second review was completed on December 19, 2007. Upon completion of the third review, a disbursement of SDR 2.0 million (6.43 percent of quota) will become available.

- The Gambia reached the HIPC completion point in December 2007, seven years after reaching the decision point, and is receiving assistance under the enhanced HIPC initiative and the Multilateral Debt Relief Initiative (MDRI).

- The previous Article IV consultation was concluded October 13, 2006. The staff report and PIN are available at http://www.imf.org/external/pubs/cat/longres.cfm?sk=20186.0. 
Executive Summary

I. Introduction .5

II. Recent Developments and Performance under the Program............................................6

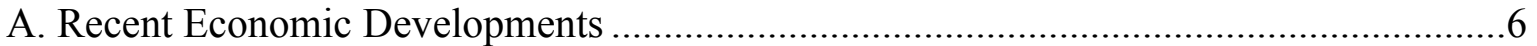

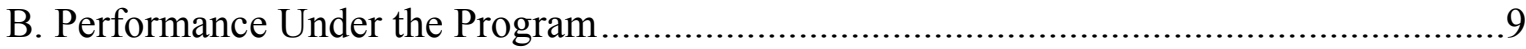

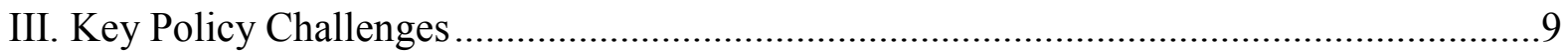

A. Coping with Rising World Food and Oil prices ................................................10

B. Maintaining External Stability ....................................................................... 10

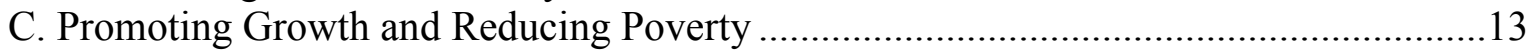

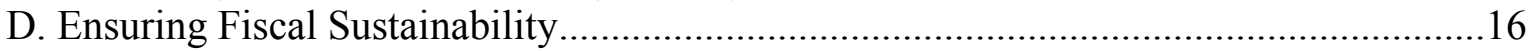

E. Making Monetary Policy More Effective.............................................................. 18

F. Enhancing Financial Intermediation ............................................................. 19

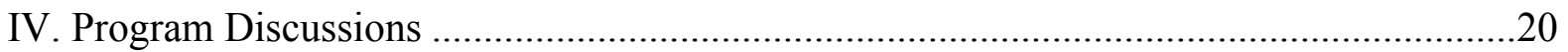

A. Medium-Term Macroeconomic Framework............................................................20

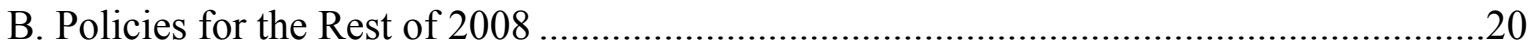

C. Program Monitoring, Risks, and Potential Augmentation .........................................21

V. Data, Fund Technical Assistance, and Financial Sector Assessment Program ................22

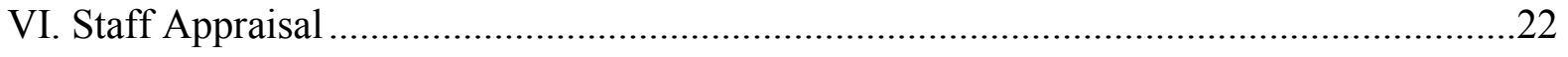

Boxes

1. Assessing External Stability in The Gambia ............................................................11

2. Survey-Based Measures of Competitiveness ............................................................ 14

Figures

1. Inflation, Exchange Rate, and Monetary Developments …..............................................

2. External Debt Indicators Before and After HIPC and MDRI Relief ...............................13

3. Progress on Selected Millennium Development Goals ................................................15

Tables

1. Selected Economic and Financial Indicators, 2004-13 ...............................................24

2. Central Government Operations, 2004-13 ...................................................................25

3. Monetary Survey, 2004-13 ..................................................................................27

4. Analytical Account of the Central Bank of The Gambia, 2004-13 ................................28

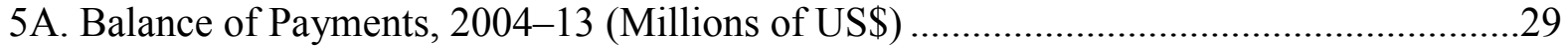

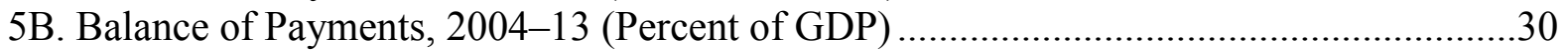

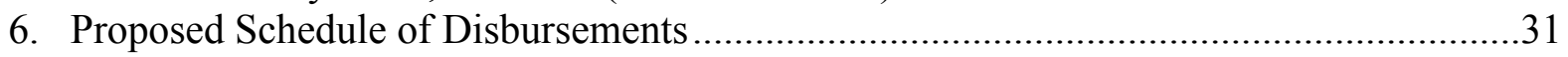

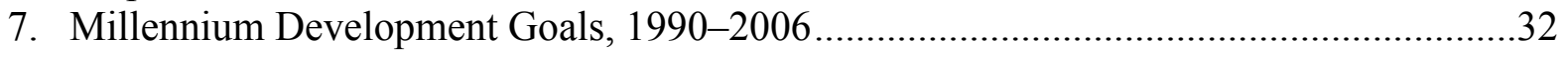


Appendix

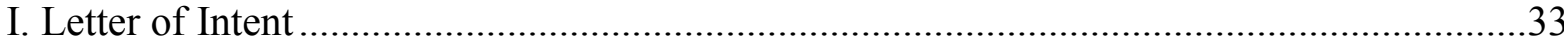

Attachment I. Memorandum of Economic and Financial Policies ..................................35

Attachment II. Technical Memorandum of Understanding ............................................47 


\section{EXECUTIVE SUMMARY}

Since the last Article IV consultation in 2006, The Gambia has maintained macroeconomic stability and made progress toward achieving debt sustainability. Fiscal performance has improved and monetary policy has been geared to maintaining low inflation. Real GDP growth has been strong and inflation has abated after a spike in the first half of 2007.

A sharp appreciation of the dalasi in 2007 has mitigated the impact of increases in world food and oil prices. The authorities' response to the continuing rise in these world prices has been measured; while eliminating sales tax on rice, they have raised other taxes to compensate for the revenue loss. Petroleum product prices have been adjusted to eliminate an implicit subsidy and bring them in line with import costs.

\section{An updated DSA confirmed the conclusion reached at HIPC completion point last December that The Gambia remains at high risk of debt distress even after receiving HIPC and MDRI assistance. To maintain external debt sustainability, staff urged the authorities to expedite the formulation of the national debt strategy and to rely mainly on grants to finance their development plans.}

Staff assesses the dalasi to be broadly in line with fundamentals. However, a marked appreciation in 2007 is likely to contribute to slower growth in 2008 through its impact on tourism. Staff and the authorities agreed that the main factors affecting The Gambia's international competitiveness include weak infrastructure, lack of access to long-term financing, and the burden of a multiplicity of taxes and local government charges.

The Gambia's revenue effort is good, but reforms are needed to make tax revenues buoyant. With the Gambia Revenue Authority now fully operational, gains from improved administration may be reaching their limit. More attention should be paid to broadening the tax base and making the tax system buoyant. The authorities agreed to review the scope for reducing import duty exemptions.

Performance under the PRGF-supported program has been satisfactory. All quantitative targets for end-March 2008 were met, and four out of eight structural performance criteria scheduled for implementation between December 2007 and July 2008 were fully met on time. In view of remedial measures undertaken by the authorities, staff supports their request for waivers for nonobservance of four structural performance criteria.

The main risks to achieving the authorities' macroeconomic objectives include continued increases in world food and oil prices, failure to maintain fiscal discipline, and shortfalls in external assistance. The authorities may seek PRGF augmentation if world food and oil prices continue to rise. Fiscal discipline is needed to contain domestic debt and put downward pressure on interest rates. Shortfalls in external assistance would undermine implementation of PRSP II. 


\section{INTRODUCTION}

1. Since the 2006 Article IV consultation, The Gambia has maintained macroeconomic stability and made progress toward achieving debt sustainability. Fiscal performance has improved, and monetary policy has been geared to maintaining low inflation. Satisfactory performance under the PRGF-supported program helped the country reach the HIPC completion point in December 2007 and thus benefit from substantial debt relief. Although debt relief has reduced The Gambia's external debt stock by more than half (from 110 percent to 50 percent of GDP at end-2007), staffs of the Fund and World Bank assess the country to be at high risk of debt distress.

2. Per capita GDP has been growing, but poverty remains widespread. Over the last five years, annual growth in per capita GDP has averaged 3.7 percent. Based on the results of the 2003 household expenditure survey, the government estimates over 60 percent of the population live below the poverty line.

3. The Gambia's second PRSP (PRSP II) provides the framework for the authorities' economic development program during 2007-11 (MEFP ף18). PRSP II reiterates the strategic priorities of the first PRSP, including: (i) macroeconomic stability and effective public resource management; (ii) promotion of pro-poor growth and employment through private sector development; and (iii) improved provision of basic services. PRSP II contains intermediate targets toward achieving the Millennium Development Goals (MDGs). The authorities have highlighted the importance of strengthened capacity in the public service for the successful implementation of PRSP II.

4. With the PRGF-supported program broadly on track, staff's discussions with the authorities focused on policies for sustaining macroeconomic stability, maintaining external stability, and boosting long-term growth. The growth discussions centered on trade and other policies to enhance The Gambia's international competitiveness. The discussions also touched on the authorities' progress in reaching their poverty reduction objectives.

5. The authorities' response to rising world food and oil prices has been measured. While reducing the sales tax on rice imports from 15 percent to 5 percent in July 2007 and eliminating it altogether in May 2008, the authorities increased other taxes (on car parts and used vehicles) to compensate for the revenue loss. The pump prices of petroleum products were increased in May 2008 by 10-24 percent to remove an implicit budget subsidy that had emerged in the preceding months. Thus, the fiscal impact has so far been limited. Appreciation of the dalasi helped cushion the impact on inflation but has lowered growth prospects for 2008 . 


\section{Recent Developments and Performance under the Program}

\section{A. Recent Economic Developments}

6. Real GDP growth has averaged about $61 / 2$ percent a year since 2004. Growth has been led by the construction, tourism and telecommunications sectors, facilitated by a steady inflow of foreign direct investment and remittances. Performance of the agriculture sector-critical for achieving the authorities' poverty reducing objectives - has been mixed. In particular, groundnut output fell substantially in the 2007/08 season largely due to a poor pattern of rainfall. At the same time, anecdotal evidence suggests increased output of nontraditional crops - mainly cashew nuts and horticultural products. The Gambia's overall growth performance compares favorably with other countries in the region.

Average Real GDP Growth and Inflation in Selected Sub-Saharan African Countries, 2004-07

\section{(Percent)}

\begin{tabular}{|c|c|c|}
\hline & Growth & Inflation \\
\hline The Gambia & 6.4 & 8.7 \\
\hline Ghana & 6.1 & 15.0 \\
\hline Malawi & 5.6 & 11.7 \\
\hline Mozambique & 7.8 & 10.7 \\
\hline Rwanda & 6.0 & 9.4 \\
\hline Senegal & 4.6 & 2.0 \\
\hline Sierra Leone & 7.2 & 11.0 \\
\hline Tanzania & 7.3 & 5.4 \\
\hline Uganda & 5.9 & 6.4 \\
\hline Zambia & 5.5 & 15.5 \\
\hline \multicolumn{3}{|l|}{ Memorandum items: } \\
\hline Sub-Saharan Africa (mean) ${ }^{1}$ & 6.2 & 9.6 \\
\hline Sub-Saharan Africa (median) & 6.0 & 10.1 \\
\hline
\end{tabular}

Source: IMF, Regional Economic Outlook, April 2008.

${ }^{1}$ For inflation, excluding Zimbabwe.

7. Inflation abated after a spike in $\mathbf{2 0 0 7}$ (Figure 1). Disruptions in the supply of foodstuffs from neighboring countries and rising import costs (e.g., rice) pushed the annual rate of inflation from less than 1 percent in December 2006 to 6-7 percent during most of 2007. However, tight monetary policy and appreciation of the dalasi helped to contain inflation pressures and lower the annual rate to 2.2 percent in June 2008.

\section{The overall fiscal balance}

improved from a 7 percent of GDP deficit in 2006 to a 0.2 percent of GDP surplus in 2007. This was achieved through a sharp fall in expenditures and net lending. Current expenditures fell by 2 percent of GDP, due primarily to a fall in domestic interest rates. Capital expenditures fell more rapidly; spending on externally financed projects fell by $5 \frac{1}{2}$ percent of GDP mainly due to a

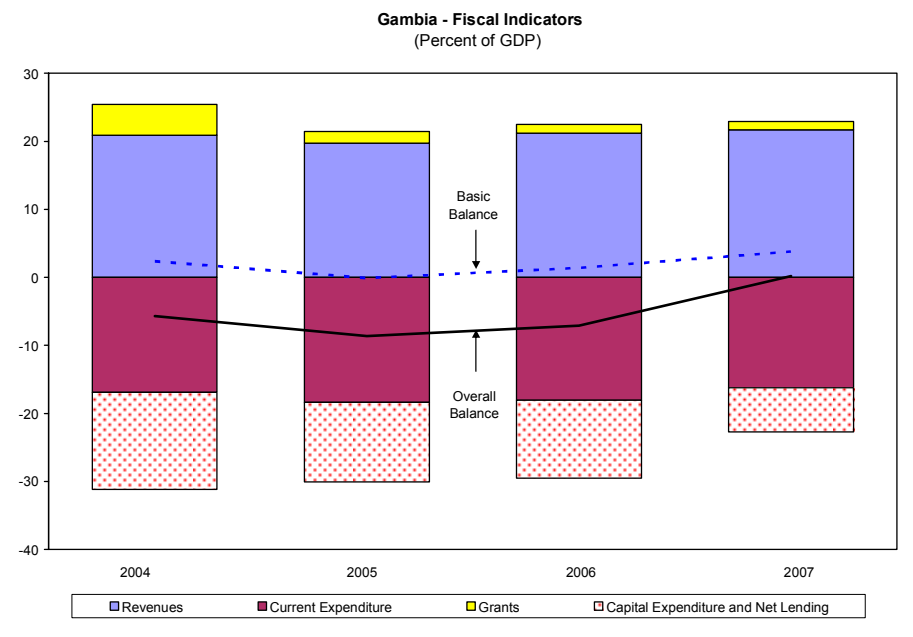
dispute between a contractor and a consultant that delayed the disbursement of grants for an (EU)-funded roads project (MEFP 94 ). The overall balance continued to be in 
7

Figure 1. The Gambia: Inflation, Exchange Rate, and Monetary Developments

After a spike in early 2007, caused by rising cost of food imports, inflation has abated...

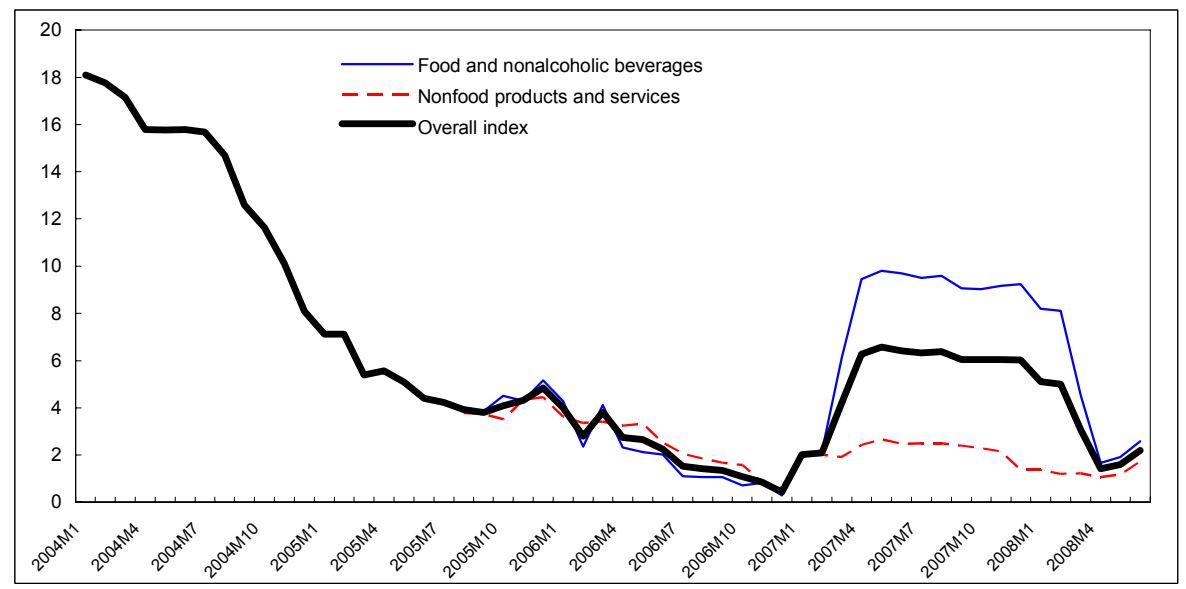

...reflecting dalasi appreciation...

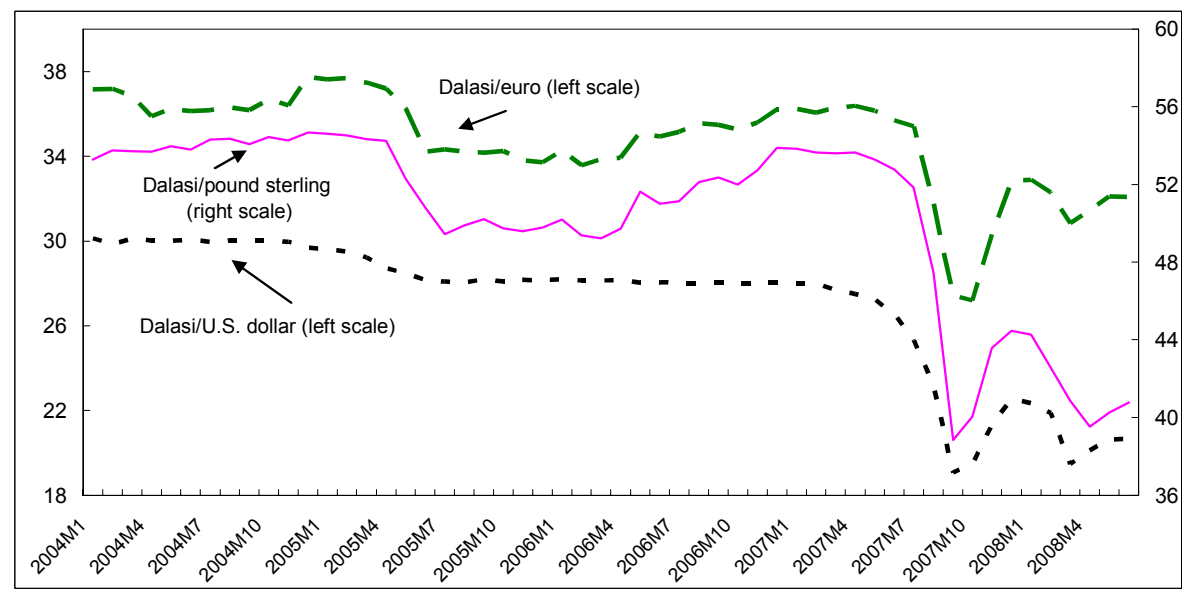

...and slowing growth in broad money.

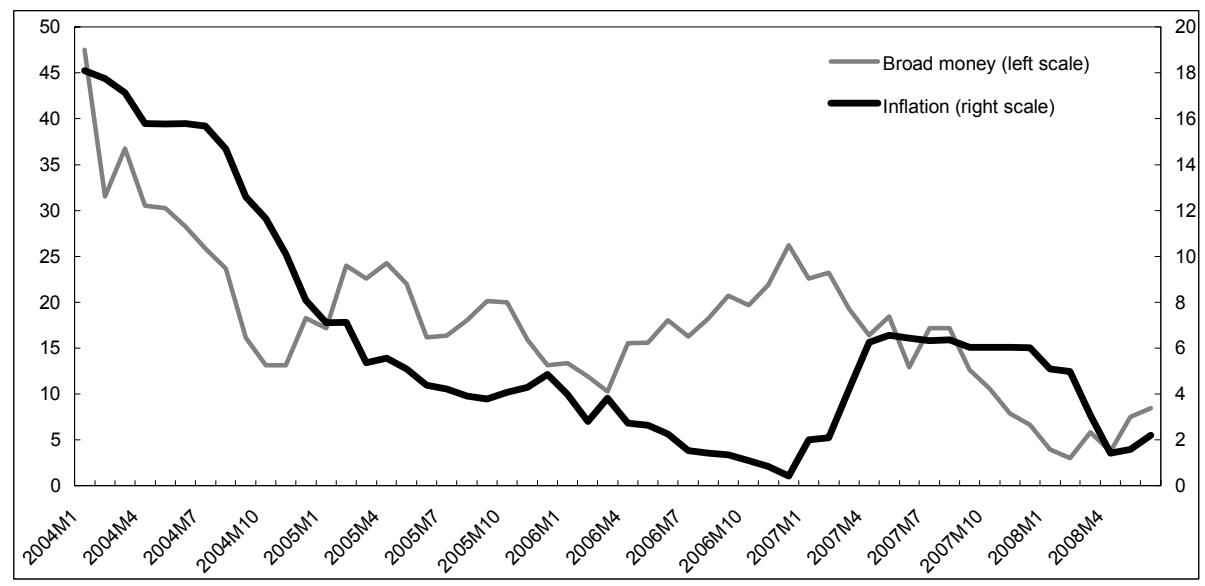

Sources: Gambian authorities and staff estimates. 
surplus in the first quarter of 2008. Increased expenditure on wages - in line with the budget and aimed at helping the government retain and attract skilled employees - has been offset by a decline in external interest payments (due to debt relief) and in externally financed capital expenditures.

\section{A significant slowdown in broad money growth in 2007 reflected improved} government finances and stagnant lending to the private sector. Broad money growth fell from 26 percent in 2006 to 7 percent in 2007. Reserve money exhibited an even more pronounced slowdown - from 24 percent expansion in 2006 to a 4 percent decline in 2007. In June 2007, to counter emerging inflationary pressures, the CBG raised its rediscount rate from 14 percent to 15 percent. In March 2008, in response to tight monetary conditions and against a backdrop of falling inflation, the CBG reduced the statutory minimum reserve requirement of banks from 16 percent to 14 percent.

10. Yields on treasury bills have fallen substantially over the last four years but commercial banks' lending rates remain sticky. Successful disinflation allowed the weighted yield on treasury bills to fall from over 25 percent in early 2005 to around 12 percent currently. By contrast, commercial banks' lending rates have been stuck above 20 percent. Banks cite high operating costs and credit risks as factors behind the high

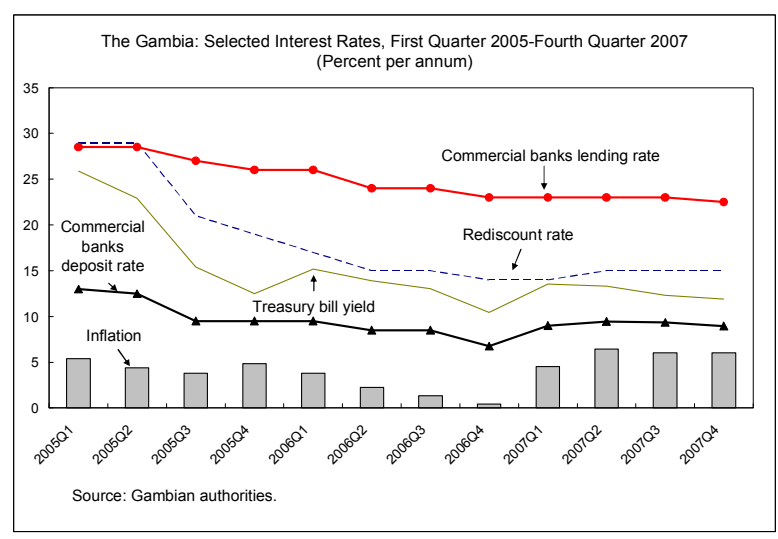
lending rates and wide interest rate spread.

\section{After remaining relatively stable from 2004 to 2006, the dalasi appreciated} significantly in $\mathbf{2 0 0 7}$ and early 2008. A sharp appreciation in the third quarter of 2007 appears to have been triggered by several banks unwinding long open foreign currency positions at the same time. Intervention by the CBG halted the appreciation and the dalasi weakened somewhat in the fourth quarter. For 2007 as a whole, the dalasi appreciated by 18 percent and 24 percent in nominal and real effective terms, respectively. The dalasi appreciated by 8 percent against the U.S.

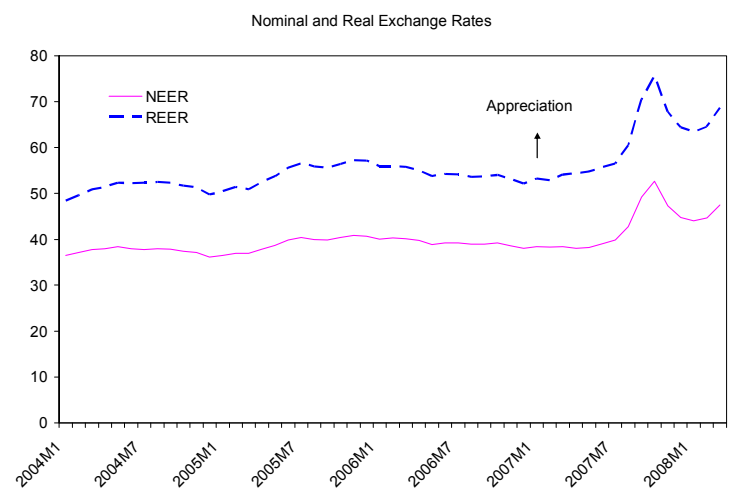
dollar in the first half of 2008, reflecting strong inflow of remittances and reduced debt service payments. 
12. The external current account deficit (including official transfers) widened from 11 $1 \frac{2}{2}$ percent of GDP in 2006 to $12 \frac{1}{2}$ percent in 2007. Current transfers (mainly remittances and official transfers) fell by 4 percent of GDP, helping push down imports by about 3 percent of GDP. The deficits were financed largely by inflows of foreign direct investment. Gross official reserves increased by US $\$ 23$ million in 2007, reaching 51/2 months of imports at year-end.

\section{B. Performance Under the Program}

13. Performance has been satisfactory. The authorities have made solid progress on fiscal consolidation, lowering interest rates and creating fiscal space for growth-promoting and poverty-reducing expenditures. The government has improved public financial management, especially budget execution, and the CBG has strengthened its governance. The authorities made less progress deepening financial intermediation and improving the quality of economic statistics.

\section{The authorities met all of the quantitative financial performance criteria for} end-March 2008 (MEFP Table A1). The cumulative basic balance through end-December 2007 fell slightly short of the indicative target, but by March 2008, it was back on target. The indicative target on new external borrowing was met, and the government is ahead of schedule on elimination of domestic budgetary arrears by end- 2008 .

\section{Four of eight structural performance criteria were implemented on time (MEFP}

Table A2). The two special audit reports on program monetary data (due at end-December 2007 and end-June 2008) were submitted to Fund staff with slight delays. The other twomaking the credit reference bureau (CRB) fully operational, and the establishment of a register of government expenditure commitments for projects largely financed by external resources - were partially implemented. The CRB has been established but concerns about the legal basis for sharing customer information have prevented it from becoming fully operational (MEFP \14). The authorities have launched a review of the Financial Institutions Act with a view to amending it to allow the CRB to share customer information among banks. With regard to the register of expenditure commitments, Department of State for Financial and Economic Affairs (DoSFEA) collected information in different formats with varying degrees of completeness (MEFP $\mid 13$ ). During the mission, the authorities designed a uniform format for compiling data on each project or loan, and the establishment of the register has now been set as a prior action for Board consideration of the PRGF third review. The authorities are requesting waivers for the structural performance criteria that were not observed.

\section{Key Policy Challenges}

16. Policy discussions addressed the following challenges to meeting the authorities' macroeconomic objectives: (a) coping with rising world food and oil prices; (b) maintaining external stability; (c) promoting growth and reducing poverty; (d) ensuring fiscal sustainability; (e) making monetary policy more effective; and (f) enhancing financial intermediation. 


\section{A. Coping with Rising World Food and Oil Prices}

17. Appreciation of the dalasi in $\mathbf{2 0 0 7}$ helped contain the impact of rising world food and oil prices on inflation. Most of the food consumed in the country is imported. The authorities indicated that available stocks of rice - the main staple — should last through September. They noted that, already, the domestic price of rice was beginning to increase and that this trend would be reinforced if world prices continue their upward trajectory and/or the dalasi weakens.

18. The impact on the balance of payments is projected to be more pronounced in 2008 than in 2007 (Table 5B). The current account is projected to worsen by almost 2 percent of GDP. An up tick in inflation is also expected in the second half of 2008.

19. To forestall pressures on the price of rice, the authorities eliminated the sales tax on rice imports and took accompanying compensating revenue measures. The mission discussed possible further measures to mitigate the impact on the most vulnerable households, including expanding existing social programs such as school feeding programs. Staff recommended the government avoid general subsidies, noting they tend to be ineffective and have created budgetary problems in other countries.

20. The mission welcomed the recent adjustment of pump prices of petroleum products to avoid a massive implicit budget subsidy. ${ }^{1}$ Staff agreed that the budget could not afford to subsidize petroleum products and urged the authorities to regularly review retail prices to keep them in line with world prices. The government intends to continue to crosssubsidize kerosene through petrol and diesel prices (MEFP $\mid 6$ ).

\section{B. Maintaining External Stability}

21. Staff assesses the value of the dalasi to be broadly in line with fundamentals. The mission discussed with the authorities the preliminary results of a Selected Issues Paper (SIP) that assesses The Gambia's external stability (Box 1). Examination of balance of payments flows suggests the current account deficit is sustainable. The external sustainability approach suggests that the exchange rate is overvalued by about 8 percent while the macroeconomic balance approach suggests overvaluation of 11 percent. The equilibrium real exchange rate approach yielded results ranging from a slight overvaluation to a significant undervaluation. The analyses are hampered by severe data weaknesses, but, on balance, staff concludes that the exchange rate is broadly in line with fundamentals. The authorities welcomed the analysis and reiterated their policy of allowing the dalasi's value to be determined in the foreign exchange market. They stressed that official intervention occurs mainly to maintain adequate international reserves or to smooth fluctuations in the exchange rate (MEFP q30).

\footnotetext{
${ }^{1}$ The price build up formula includes an excise tax and a sales tax, as well as a residual element that operates like a virtual stabilization fund. A positive residual is interpreted as a windfall tax, while a negative residual is considered an implicit subsidy.
} 


\section{Box 1. Assessing External Stability in The Gambia}

Different approaches to assessing The Gambia's external stability yield different results. The external sustainability (ES) approach indicates a real depreciation of about 8 percent would be required to stabilize the NFA to GDP ratio at its current level while the macroeconomic balance approach suggests a depreciation of 11 percent is needed. The equilibrium real exchange rate (ERER) approach suggests a range of results from a 2-percent overvaluation to 33-percent undervaluation in 2007.

The Balance of Payments and REER. Except for a sharp fall in reserves in 2001, the overall balance of payments has registered surpluses averaging 2 percent per year since 1997. The current account deficit increased substantially beginning in 2004. However, this was caused by additional investment in the tourism and telecommunication sectors. After the switch to a floating exchange rate regime in 1986, the REER remained within 10 percent of its average level until 2000. A burgeoning fiscal deficit and loose monetary policy caused the REER to fall 50 percent between 2000 and 2003. Fiscal consolidation and tight monetary policy allowed the REER to stabilize from 2003 to 2007. Comparison with other countries in the region indicates the fall in the REER is consistent with lower productivity growth in The Gambia.

Underlying current account, external sustainability and macroeconomic balance approaches. The underlying current account balance is defined as the observed current account balance stripped of temporary factors. The underlying current account deficit is estimated at 11.7 percent of GDP in 2007 compared to the actual deficit of 12.5 percent of GDP.

The external sustainability approach calculates the current account balance that stabilizes the NFA to GDP ratio at today's level. As a result of debt relief, The Gambia's net foreign liabilities ratio fell from just under 200 percent of GDP during 2003-2006 to 56 percent of GDP at end-2007. Staff calculates the current account deficit that would stabilize the NFA ratio - given projected average nominal growth of 10 percent-at 5.9 percent of GDP. Based on estimated elasticities, The Gambia's real exchange rate would need to depreciate by about 8 percent to bring the underlying current account deficit down to the level needed to stabilize the NFA-to-GDP ratio. The macroeconomic balance approach estimates a current account norm based on The Gambia's economic fundamentals compared with other countries. This approach indicates a current account norm of 4 percent of GDP is appropriate for The Gambia, requiring a 11 percent depreciation.

Equilibrium real exchange rate (ERER). A single-country estimation indicates a very small overvaluation of the dalasi at end-2007. A panel regression using coefficients from 28 oil-importing sub-Saharan African countries ${ }^{2}$, indicates the real exchange was 33 percent below its fundamental value in 2007, implying that economic fundamentals cannot explain the drop in the dalasi between 2000 and 2003. The external terms of trade for goods and services, productivity relative to trading partners (defined as relative per capita real GDP), and government consumption relative to trading partners yield statistically significant estimations for both single country and panel regressions. Statistical significance notwithstanding, the outcome of this approach is subject to data uncertainties and a relatively short sample period (only 19 annual observations for estimating the REER using single-country estimation techniques).

Single-country estimation of ERER

Panel estimation of ERER

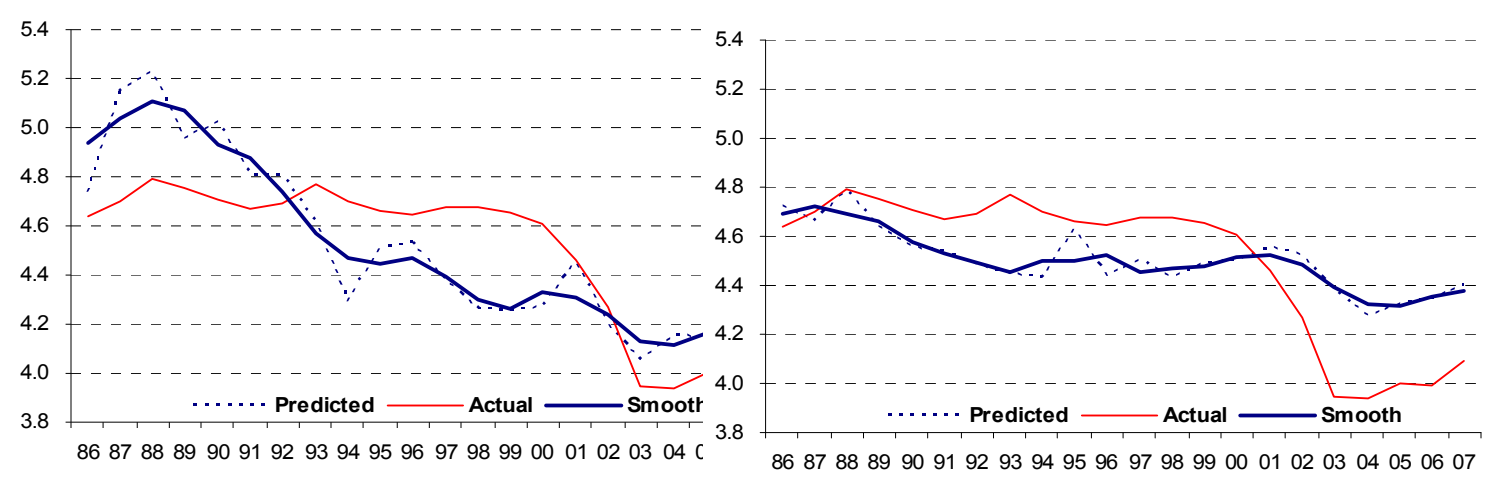

${ }^{2}$ Chudik and Mongardini (2007). 


\section{The Gambia remains at high risk of debt distress because of the high level of} outstanding debt and the country's vulnerability to shocks. An update of the LIC DSA undertaken by staff found that lower-than-expected external borrowing in 2007 and thus far in 2008 has improved key debt ratios. ${ }^{3}$ Thus, the NPV of debt-to-GDP ratio now falls comfortably below the threshold of 30 percent over the 20 year projection horizon. However, the NPV of debt-to-exports ratio still peaks at 138 percent of GDP, well above the threshold of 100 percent.
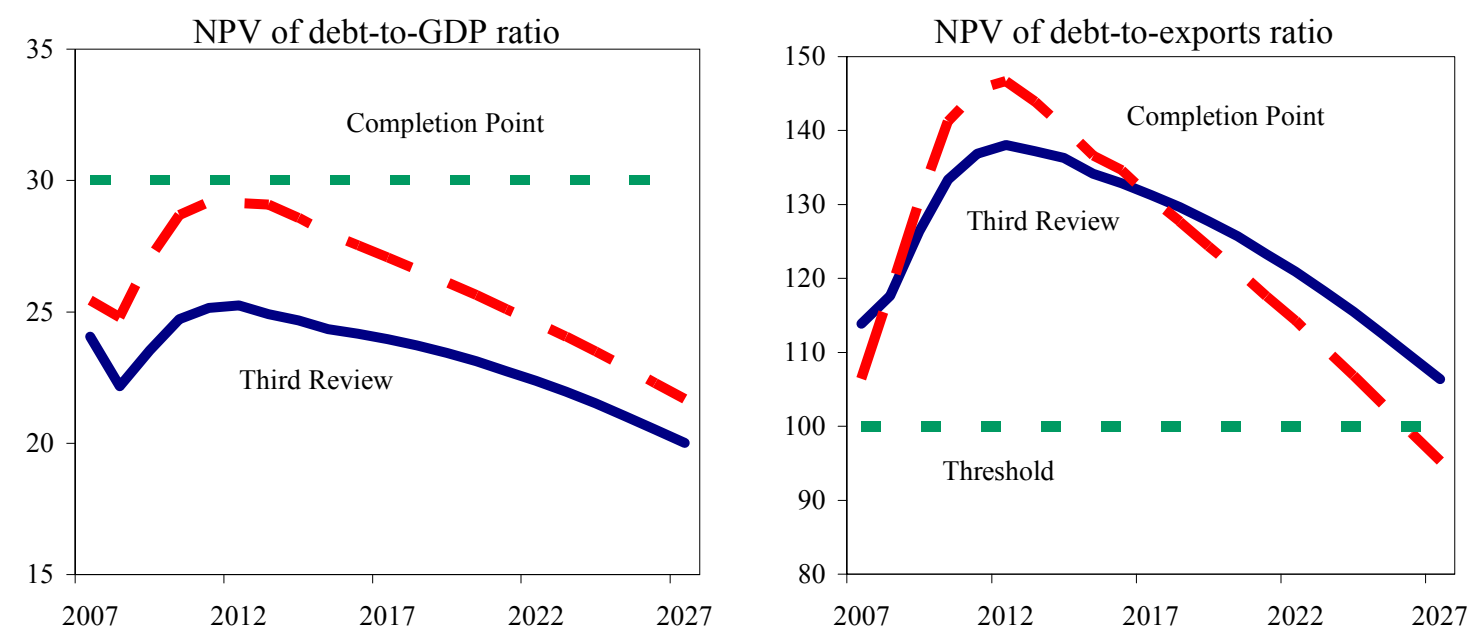

23. The authorities indicated that they were awaiting assistance from the Commonwealth Secretariat to undertake an independent DSA which would provide input to their national debt strategy. Staff urged the authorities to expedite the formulation of their strategy and to rely mainly on grants to finance their development plans. Staff noted that The Gambia's debt and debt service ratios are among the highest in the group of countries that have recently reached the HIPC completion point and benefited from MDRI assistance (Figure 2). The authorities responded they expect to have the debt strategy in place by end-February 2009, will ensure that new loans have a minimum grant element of 45 percent, and will respect the indicative limit on new borrowing for 2008 (MEFP q33).

\footnotetext{
${ }^{3}$ The lower than expected external borrowing was the result of a slower than expected pace concluding negotiations with lenders. The debt sustainability assessment is presented in Supplement 1.
} 


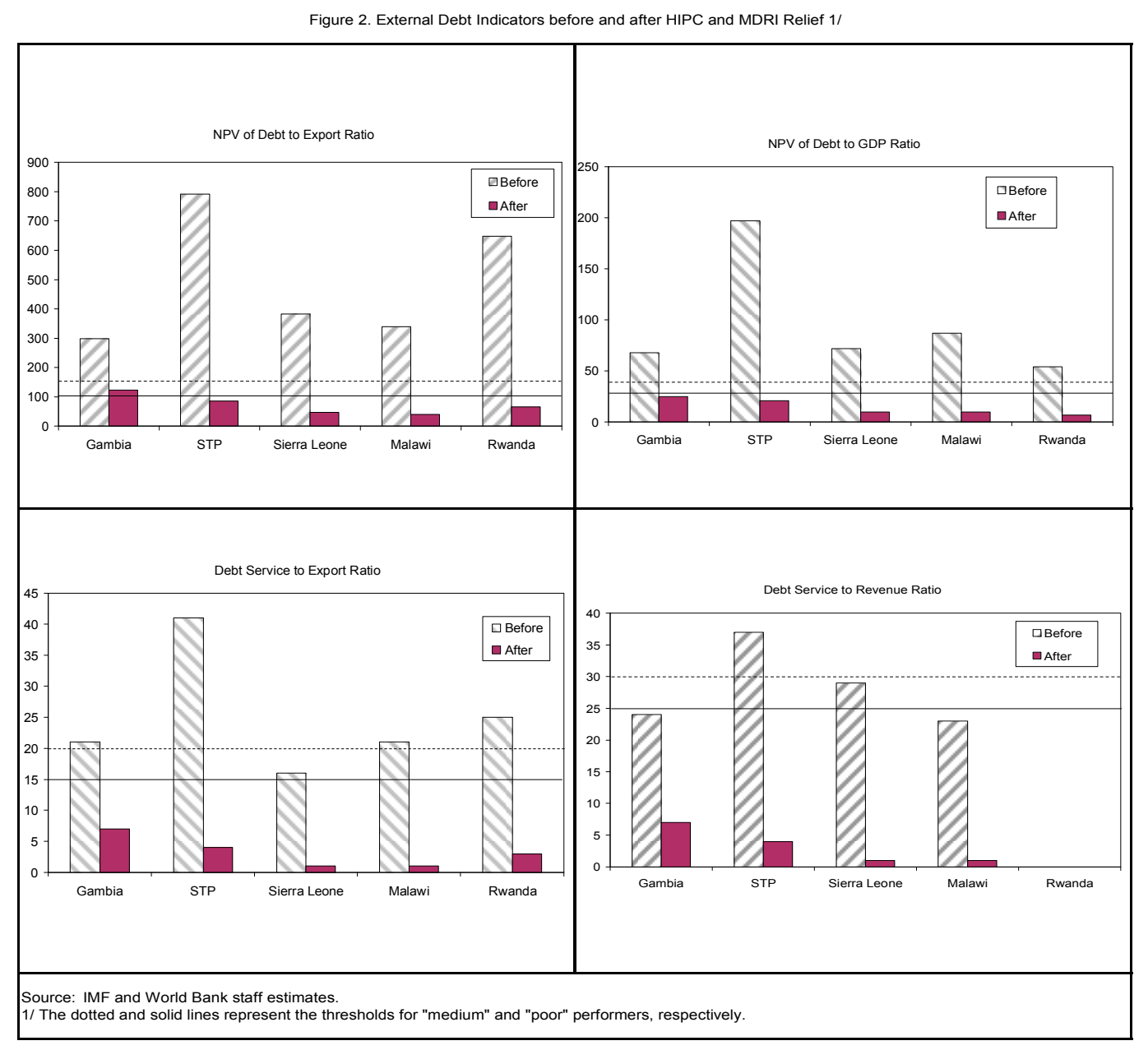

C. Promoting Growth and Reducing Poverty

\section{Enhancing the country's international competitiveness is central to the}

authorities' strategy for promoting growth. Surveys and studies suggest a broad agenda of reforms is needed to address structural constraints to growth. The Gambia ranked 131 out of 178 in the World Bank's Doing Business Indicators for 2008 and 102 out of 131 countries in the World Economic Forum's Competitiveness Indicators for 2007-08 (Box 2). The Gambia ranked highly on employing workers, trade, and enforcing contracts. Problem areas included paying taxes, protecting investors, innovation, health, and education. A 2006 Investment Climate Assessment done by the World Bank found that firms ranked electricity, access to credit, land access, and a heavy tax burden as the most important obstacles to doing business in the country. A Diagnostic Trade Integration Study led by the World Bank and completed in 2007, concluded that The Gambia's role as a regional entrepot was eroding, and made wide ranging recommendations to boost the country's international competitiveness (MEFP 『19). The mission noted that most of the recommendations of the various studies fall outside the remit of the Fund, but highlighted tax policy as an area where the Fund could offer assistance. 


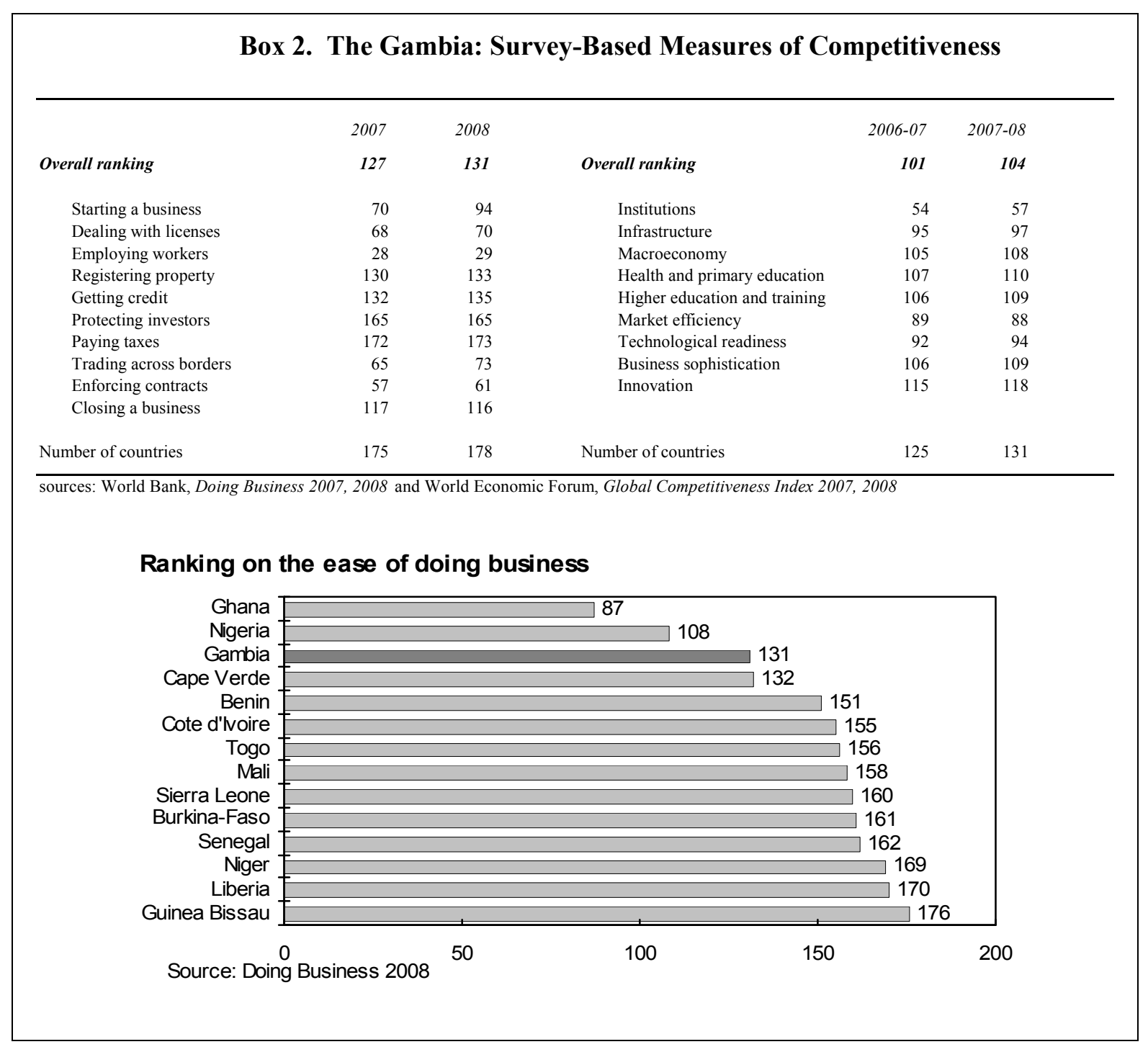

\section{In addition to promoting pro-poor growth, the authorities are committed to} boosting poverty-reducing expenditures in the budget. The PRSP specifies that 25 percent of government revenues and 30 percent of departmental budgets should be spent on priority sectors including agriculture, education, health, and the environment. The ratio of priority spending to government revenues has risen but remains below 21 percent. The government achieved the target for the departmental budgets in 2005 and 2006. 

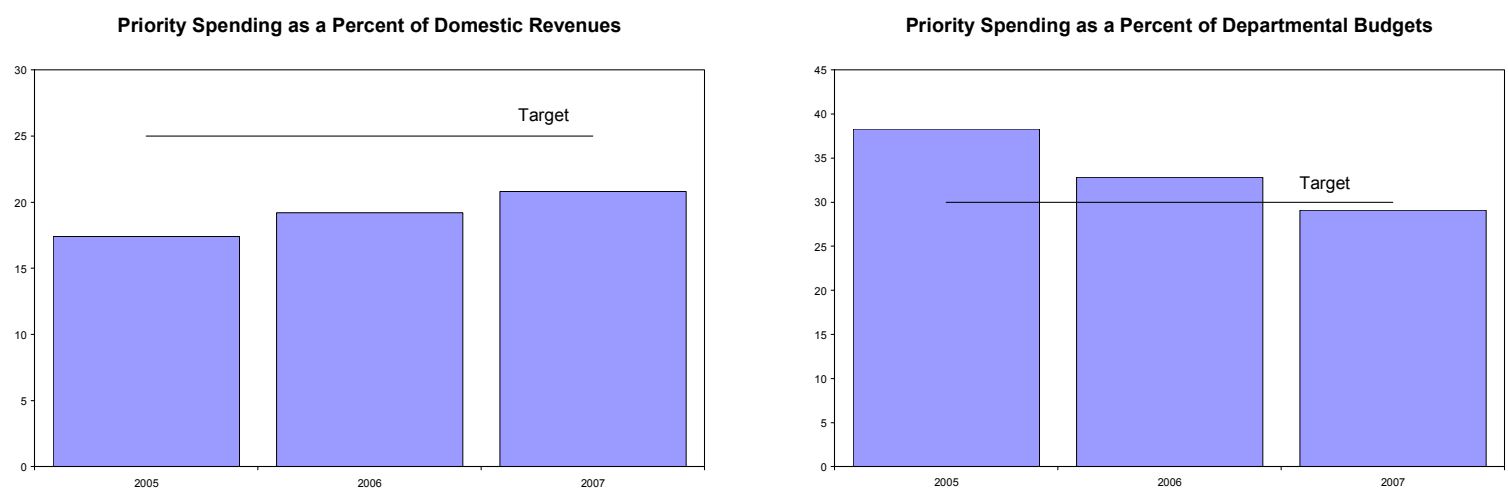

26. Progress towards the Millennium Development Goals has been mixed (Figure 3 and Table 7). The Gambia has achieved gender equality as measured by the female to male primary school enrollment ratio and made significant progress on boosting primary school enrollments. Primary and secondary health care have expanded significantly and increased immunization has reduced mortality rates. However, the incidence of poverty has not declined, undernourishment is still a serious problem, and the incidence of malaria and tuberculosis remain high.

Figure 3. The Gambia: Progress on Selected Millennium Development Goals

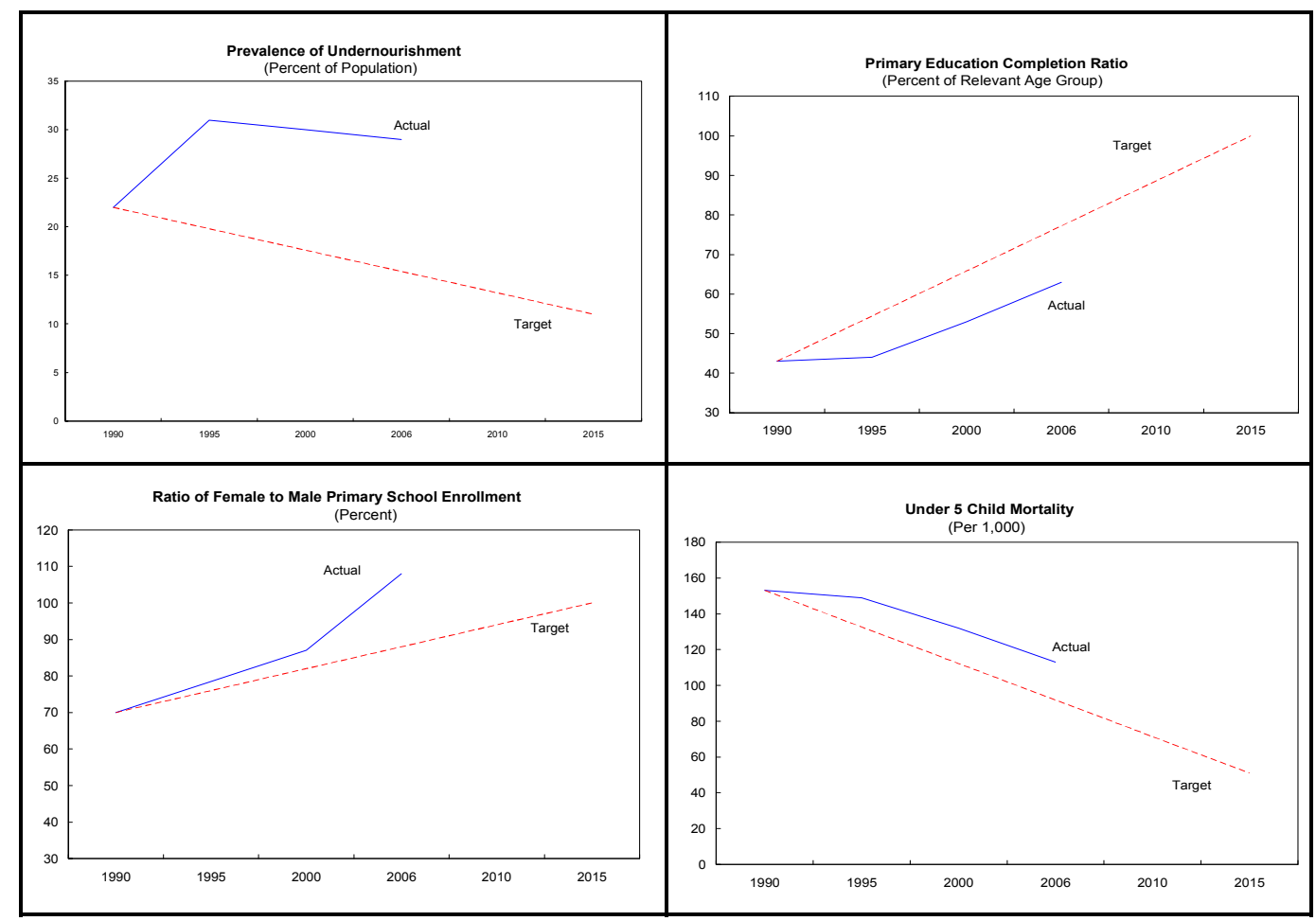

Source: World Bank Development Indicators. 
27. Financial and technical assistance from The Gambia's development partners will be critical for the successful implementation of PRSP II. PRSP II highlights capacity weaknesses, policy slippages, and shortfalls in the delivery of aid as important factors in the poor outcomes under the first PRSP. The government presented PRSP II to a Donor Round Table Conference in February 2008, and spearheaded the establishment of an in-country Development Partners Coordination and Consultation Mechanism (MEFP q35-q37). The incountry forum will be used to monitor and analyze progress using a Results Matrix that contains quantitative indicators and targets matched to PRSP objectives.

\section{Ensuring Fiscal Sustainability}

28. Continued fiscal consolidation will enable the authorities achieve public debt sustainability (see section III of Supplement 1). Overall fiscal deficits are projected to average 2.7 percent of GDP during 2008-13. With external financing averaging $3 \frac{1}{2}$ percent of GDP and domestic surpluses measuring $3 / 4$ percent of GDP, domestic debt falls by more than half from its end-2007 level of 28 percent of GDP. This allows room for some increase in domestic borrowing in the event of a shortfall in external assistance.

29. At about 21 percent of GDP, The Gambia's revenue effort is good, but reforms are needed to make tax revenues buoyant. Improvements in revenue administration have been important in boosting government revenues over the last four years. Reforms included the creation of the Gambia Revenue Authority (GRA), establishment of a large taxpayer unit, and the introduction of taxpayer identification numbers. With the GRA now fully operational, gains from improved administration may be reaching their limit. More attention should be paid to broadening the tax base and making the tax system buoyant. The lack of buoyancy is explained by the dominance of import taxes in revenues and by the increasing proportion of imports exempt from customs duties. The authorities agreed to review the scope for reducing these exemptions.

The Gambia: Dutiable and Non-Dutiable Imports, 2004-07

\begin{tabular}{|c|c|c|c|c|}
\hline & 2004 & 2005 & 2006 & 2007 \\
\hline & & ent of $t$ & & \\
\hline Dutiable & 85 & 81 & 74 & 74 \\
\hline Non-petroleum & 74 & 68 & 67 & 64 \\
\hline Petroleum & 11 & 13 & 7 & 10 \\
\hline Nondutiable & 15 & 19 & 26 & 26 \\
\hline Government & 3 & 3 & 12 & 11 \\
\hline Investment code & 8 & 9 & 10 & 11 \\
\hline Diplomatic & 2 & 4 & 3 & 2 \\
\hline Other duty free & 3 & 3 & 2 & 2 \\
\hline Total & 100 & 100 & 100 & 100 \\
\hline
\end{tabular}




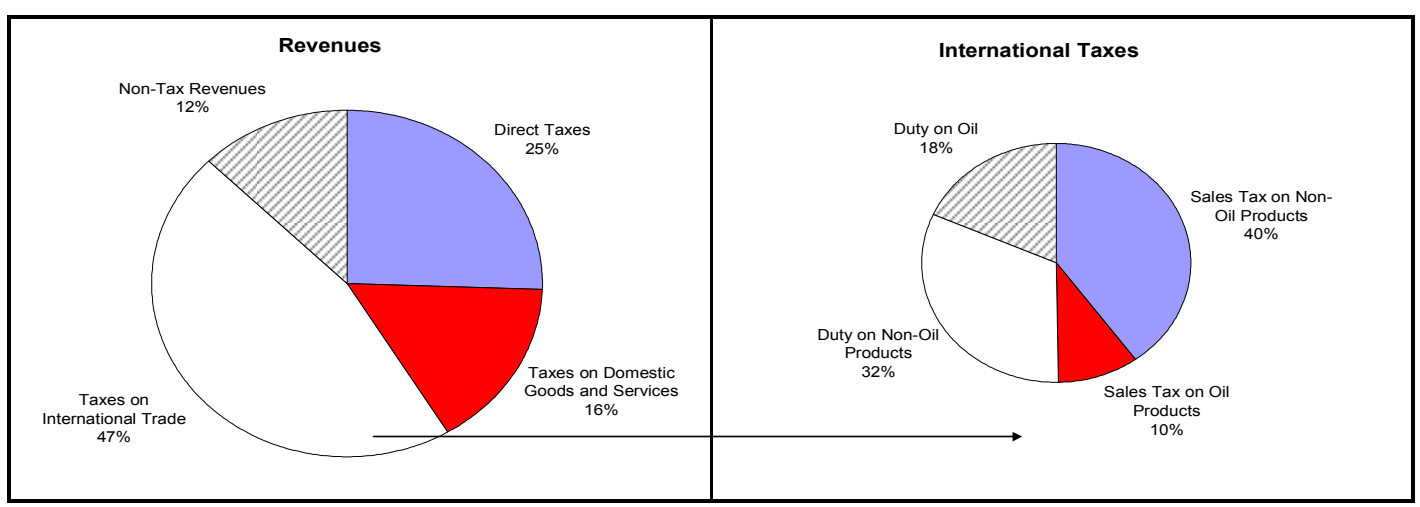

30. The fiscal impact of an Economic Partnership Agreement (EPA) with the EU should be manageable. The Gambia is involved in ongoing negotiations with the EU as part of the Economic Community of West African States. The mission presented the preliminary results of an empirical analysis of the potential revenue loss from an EPA, and advised the authorities to pursue commitments by the EU to compensate partners for net revenue losses. ${ }^{4}$ The analysis suggests that:

- $\quad$ A full and immediate liberalization of trade with the EU would lead to revenue losses of about $2 \frac{1}{2}$ percent of GDP a year and represent a major fiscal shock to The Gambia.

- However, a gradual trade liberalization that takes advantage of the allowance of protection for 20 percent of imports from the EU and of a long transition period would contain the revenue loss to a $1 / 2$ percent of GDP a year over the first five years.

31. The government is considering options for a comprehensive civil service reform program, drawing on studies funded by the World Bank and other donors. It is seeking to build the capacity of the civil service to formulate and implement policies and to deliver public services efficiently. Over time, low pay and difficult working conditions have led to an exodus of professional and managerial staff which has severely undermined the functioning of the service. The reform program is expected to cover pay and benefits, career development, and job security issues (MEFP q23). On the basis of the available analytical studies, staff believes the macroeconomic framework can support a substantial increase in the wage bill. However, staff emphasized that the pay reform should be targeted to retaining and attracting professional and managerial staff. The authorities agreed, but said increases will be needed at lower grades too to move them towards a living wage. The government will decide on the details of the pay reform as part of the preparation of the 2009 budget.

\section{Introduction of the Integrated Financial Management Information System} (IFMIS) has improved expenditure management, but more reforms are needed to strengthen public financial management. In particular, the process of budget formulation needs to be strengthened to ensure that budget allocations are realistic and in line with

\footnotetext{
${ }^{4}$ See SIP chapter on fiscal implications of an economic partnership agreement.
} 
priorities. The establishment of a register to track government's own commitments for capital projects funded largely by donors is a key part of structural reforms under the PRGFsupported program.

\section{E. Making Monetary Policy More Effective}

33. Monetary operations are guided by a money targeting framework, using broad money as the intermediate target and reserve money as the operating target. Weekly treasury bill auctions are the CBG's main instrument for achieving its target. The CBG uses a shortterm liquidity forecasting framework to determine weekly monetary operations. Announcements about the rediscount rate at the end of bimonthly Monetary Policy Committee meetings are also used to signal its policy stance.

\section{A high degree of uncertainty about monetary policy transmission mechanisms}

hampers monetary operations. The primary objective of monetary policy in The Gambia is price stability, and the CBG has done a good job of reining in inflation. However, a recent MCM technical assistance mission noted that the shallowness of the money market, and an environment of uncertainty about the stability, persistence and relative importance of the possible channels of monetary transmission constrain monetary policy. The mission discussed with the CBG the findings of the MCM mission as well as staff analysis that suggests that the demand for broad money may not be stable, thus casting doubt on its appropriateness as nominal anchor for monetary policy. In this regard, the mission suggested that CBG supplement its money targeting framework with a range of indicators (including the outlook for inflation and exchange rate developments).

\section{The almost exclusive reliance on treasury bills for monetary operations has} generated conflicting signals about monetary policy. Treasury bills have been effective at mopping up liquidity when the authorities have been willing to let interest rates rise (and signaled that willingness by raising the rediscount rate). However, the CBG's ability to inject liquidity through the treasury bill market has been constrained by low balances in the sterilization account. Hence, on occasion, even though the liquidity forecasting framework called for injecting liquidity, the $\mathrm{CBG}$ issued new bills to rollover maturing bills. Staff recommended the CBG consider foreign exchange operations as an additional instrument to influence dalasi liquidity. In the judgment of staff, there is scope for higher growth in reserve money (closer to program projections) without re-igniting

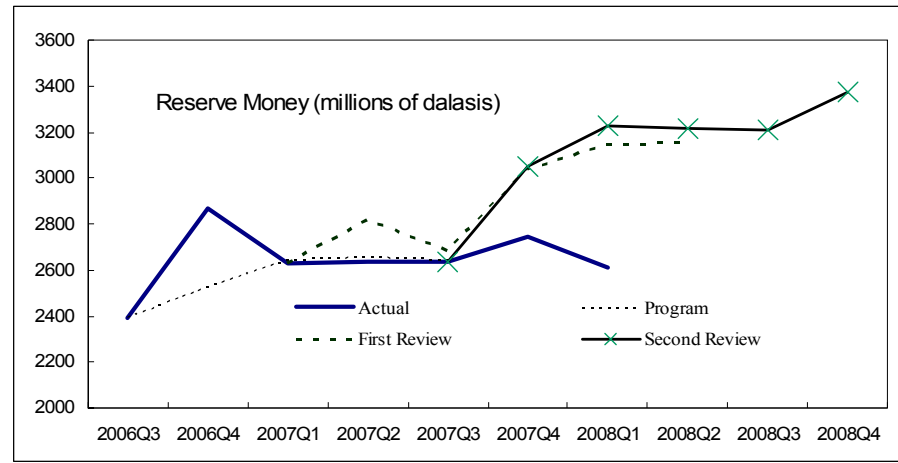
inflation.

36. The mission discussed a work program for enhancing the monetary policy framework. The main elements are: (i) enhancing short-term liquidity forecasting by 
improving projections of government revenues and expenditures; (ii) carrying out analytical work to better understand the transmission mechanism of monetary policy; (iii) developing leading indicators of economic activity; and (iv) improving data quality, especially on output and interest rates of commercial banks.

\section{F. Enhancing Financial Intermediation}

37. The financial sector is sound and competition has been increasing. The entry of two new banks in 2007 brings the number of commercial banks operating in The Gambia to ten. The banks are generally profitable, adequately capitalized, and highly liquid, although profitability and liquidity ratios fell during the third quarter 2007 as banks suffered valuation losses associated with the steep appreciation of the dalasi. Interest rate spreads are high, though not out of line with levels in other countries in sub-Saharan Africa. Nonperforming loans, at 12-14 percent, are moderate by regional standards. However, the loan-to-deposit ratio (41 percent) is relatively low in part due to high risks associated with a weak legal system (MEFP \10).

\begin{tabular}{|c|c|c|c|c|}
\hline \multicolumn{5}{|c|}{$\begin{array}{l}\text { Selected Sub-Saharan African Countries: } \\
\text { Selected Financial Sector Indicators, } 2008 \text { 1/ }\end{array}$} \\
\hline & $\begin{array}{l}\text { Credit/Deposit } \\
\text { (Percent) }\end{array}$ & $\begin{array}{l}\text { Interest Rate Spreads } \\
\text { (Percent) }\end{array}$ & $\begin{array}{l}\text { Nonperforming Loans } \\
\text { (Percent of total loans) }\end{array}$ & $\begin{array}{l}\text { Broad Money/GDP } \\
\text { (Percent) }\end{array}$ \\
\hline The Gambia & 40.5 & 13.5 & 13.0 & 51.7 \\
\hline Benin & 75.8 & & 10.4 & 29.8 \\
\hline Ghana & 64.5 & 15.3 & 7.0 & 36.3 \\
\hline Malawi & 84.8 & 22.1 & 2.6 & 14.8 \\
\hline Mozambique & 56.5 & 7.3 & 3.7 & 32.3 \\
\hline Rwanda & 87.3 & 7.6 & 32.0 & 21.8 \\
\hline Senegal & 85.6 & 8.5 & 18.8 & 36.6 \\
\hline Sierra Leone & 32.7 & 15.3 & 31.7 & 23.3 \\
\hline Tanzania & 61.8 & 5.9 & 6.3 & 26.9 \\
\hline Uganda & 60.2 & 8.1 & 3.4 & 15.0 \\
\hline Zambia & 52.5 & 12.0 & 11.5 & 18.0 \\
\hline Average 2/ & 66.2 & 11.3 & 12.7 & 25.5 \\
\hline
\end{tabular}

Source: IMF, International Financial Statistics and Monthly Country Briefs.

1/ Most recent data available (2007 for interest rate spreads and early 2008 for others). 2/ Excluding The Gambia.

38. Reforms are underway to deepen financial intermediation. Making the Credit Reference Bureau operational should reduce lending risks. The authorities are also reviewing several laws (including the Financial Institutions and Mortgage Acts) to remove legal impediments to financial intermediation. The $\mathrm{CBG}$ reported progress in strengthening capacity in banking supervision (with technical assistance from the Fund), and in implementing the Prompt Corrective Action framework introduced in July 2007 to monitor the health of banks. After the sharp appreciation of the dalasi in late 2007, the CBG has 
tightened enforcement of prudential regulations on banks' foreign currency net open positions. The CBG decided to lower banks' reserve requirements partly to lower the cost of funds to the banks and lower barriers to financial intermediation.

\section{Program Discussions}

\section{A. Medium-Term Macroeconomic Framework}

39. Revisions to the medium-term macroeconomic framework (Tables 1-5) take account of the changes in the international environment and staff's reassessment of the outlook for external financing (including new budget support from the World Bank amounting to about US\$7 million in 2009). The main features of the updated framework compared to the second review are: (i) a downward revision in GDP growth of about 1 percent in 2008 and $1 / 2$ percent in 2009 due to the impact of global downturn and dalasi appreciation on tourism and remittances and poor output in the groundnut sector; (ii) inflation about one percent higher in 2008 - due to the pass through of higher food and energy prices - but returning to a downward path in 2009; (iii) maintenance of the surplus on the fiscal basic balances for 2008 and 2009 of about 1 1/2 percent of GDP but lower interest rates leading to a more rapid decline in domestic debt; (iv) broad money growth of 12 percent, slightly higher than nominal GDP growth; and (v) a deterioration in the current account balance including official transfers in 2008 due to higher world food and oil prices.

\section{B. Policies for the Rest of 2008}

40. The updated macroeconomic framework envisions that the central government basic balance will remain in surplus of about $1 \frac{1}{2}$ percent of GDP while the overall deficit drops to 3 percent of GDP in 2008. The improvement in the overall balance compared to the second review is attributable to a downward revision in externally financed capital expenditures (in line with the revised outlook for external grants and loans). Domestic revenues are projected to remain at 21 percent of GDP with a small drop in personal and corporate taxes offset by increased revenues from the sales tax on oil imports. On the expenditure side, current expenditures are expected to remain at about 16 percent of GDP while capital expenditures fall from 13 to 10 percent of GDP. Domestic interest bearing debt is projected to fall from 28 percent of GDP at end-2007 to $22 \frac{1}{2}$ percent of GDP at end-2008.

41. The CBG indicated monetary policy will continue to aim at keeping inflation low. Rising world food and oil prices will pose a challenge. Improved coordination of fiscal and monetary policies will help meet the challenge.

42. The government is seeking debt relief from all its creditors. Following a Paris Club agreement in January 2008, the government has signed bilateral debt relief agreements with France and Norway, and expects to sign agreements with its two other Paris Club creditors (Austria and Netherlands) in the coming months. Kuwait has agreed to provide debt 
relief on comparable terms to those of the Paris Club. The authorities are following up their previous communications with other creditors.

\section{Program Monitoring, Risks, and Potential Augmentation}

43. The program's quantitative targets have been extended through June 2009 (MEFP, Table A1) while the target dates for implementing some structural measures have been reset. In particular, to allow adequate time for establishing a legal basis for the $\mathrm{CRB}$ to share information on customers among banks, the performance criterion on making the CRB operational has been reset from end-March 2008 to end-March 2009 (MEFP $\mid 14$ ). The Auditor-General delayed the submission of the report on the government's 2000-04 accounts to the national assembly to give the Treasury Directorate an opportunity to respond to some queries and correct portions of the accounts. The final report is now scheduled to go to the national assembly in September 2008 (MEFP $\uparrow 16)$.

44. Structural conditionality has been streamlined. The broad areas of focus remain unchanged: (i) public financial management and accountability; (ii) central bank governance; (iii) financial intermediation; and (iv) economic statistics. In recognition of progress made to date, conditionality in the first two areas has been streamlined. There is no longer a performance criterion on monthly fiscal reporting, which has now become routine. The requirement of special audit reports of monetary data submitted to staff by the CBG for each test date has also been dropped. These reports have served their purpose of addressing reporting weaknesses.

\section{The main risks to achieving program objectives include:}

- Continued increases in world food and oil prices. These would fuel inflation and worsen the current account deficit.

- Failure to maintain fiscal discipline. This would lead to larger fiscal deficits, higher levels of public debt, and higher domestic interest rates.

- Sharp movements in the exchange rate. An appreciation would likely hurt tourism and export earnings, while a depreciation would add to inflation pressures and worsen the burden of external debt service.

- Shortfalls in external assistance. These would jeopardize the program's growth and poverty-reduction objectives. For debt sustainability reasons external assistance should be mainly in the form of grants or very highly concessional loans.

46. The authorities may request PRGF augmentation at the next review if world oil and food prices continue to rise (MEFP $\uparrow 41)$. 


\section{Data, Fund Technical Assistance, and Financial Sector Assessment PROGRAM (FSAP)}

47. Statistical data are broadly adequate for surveillance, but substantial weaknesses remain in real sector and balance of payments. The national accounts data are compiled using only the production approach, although progress has been made in developing expenditure data. Implementation of an IFMIS system has improved fiscal reporting; consolidated budget reports are now produced monthly with a one month lag. Balance of payments reporting still has several gaps, in particular for re-exports and service income. Data on private capital flows are poor but official grant and loan disbursements are relatively well recorded.

48. The authorities welcomed the appointment of a regional fiscal adviser to assist with public financial management. The adviser, based in Liberia, made his first visit to Banjul in July 2008. The authorities have requested ongoing assistance on banking supervision, statistics (national accounts, balance of payments, and financial), and compilation of a producer price index.

49. The authorities also requested that The Gambia be considered for an FSAP (MEFP १44).

\section{Staff Appraisal}

50. The authorities are to be commended for maintaining macroeconomic stability and achieving robust growth. This resulted from prudent economic policies, including significant fiscal consolidation and monetary policy geared to maintaining low inflation.

51. Staff welcomes the authorities' measured response to rising world food and oil prices. So far, appreciation of the dalasi has helped contain the impact inflation but this is likely to change in 2008. The authorities should continue to pass through increases in world prices while considering targeted measures to help the poor, including expanding existing social programs such as school feeding programs.

52. Staff assesses the dalasi to be broadly in line with fundamentals. Different empirical approaches yielded significantly different results — ranging from slight overvaluation to substantial undervaluation. The analyses are hampered by severe data weaknesses, but, on balance, staff concludes that the exchange rate is broadly in line with fundamentals.

53. Notwithstanding some improvement in debt indicators, The Gambia remains at high risk of debt distress. To the extent possible, the authorities should rely on grants and highly concessions loans for financing their development program. In this regard, staff welcomes the authorities' efforts to strengthen their engagement with donors in order to mobilize resources for implementing PRSP II. 
54. Further efforts are needed to improve the investment climate and The Gambia's international competitiveness. Staff encourages the authorities to work with the World Bank and other donors for the speedy implementation of the recommendations of the DTIS, especially in relation to the business climate.

55. Reforms are needed to make the tax system buoyant. The government has made important progress on tax administration and expenditure management, but further reforms are needed to broaden the tax base and make it more buoyant. In particular, staff urges the authorities to reduce the scope of import duty exemptions.

56. Staff believes there is scope for higher growth in reserve money without fueling inflation. In view of persistent undershooting of the CBG's reserve money path, staff recommends the use of foreign exchange operations as an additional instrument to influence dalasi liquidity.

57. Staff supports the authorities request for waivers for the nonobservance of four structural performance criteria. The delays in submitting the two special audit reports were due to circumstances beyond the authorities' control. The authorities have initiated steps toward removing the legal obstacles to making the CRB fully operational, and the establishment of the register of government expenditure commitments is now a prior action for completing the third PRGF review.

58. Staff recommends that the next Article IV consultation take place within 24 months, in line with the Decision on consultation cycles in program countries. 
Table 1. The Gambia: Selected Economic and Financial Indicators, 2004-13

\begin{tabular}{|c|c|c|c|c|c|c|c|c|c|c|c|c|}
\hline & \multirow{2}{*}{$\frac{2004}{\text { Act. }}$} & \multirow{2}{*}{$\frac{2005}{\text { Act. }}$} & \multirow{2}{*}{$\frac{2006}{\text { Act. }}$} & \multicolumn{2}{|c|}{2007} & \multicolumn{2}{|c|}{2008} & \multirow{2}{*}{ 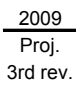 } & \multirow{2}{*}{ 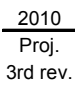 } & \multirow{2}{*}{ 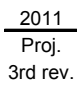 } & \multirow{2}{*}{ 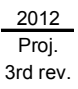 } & \multirow{2}{*}{$\begin{array}{c}2013 \\
\text { Proj. } \\
\text { 3rd rev. }\end{array}$} \\
\hline & & & & $\begin{array}{c}\text { Proj. } \\
\text { 2nd rev. }\end{array}$ & Act. & $\begin{array}{l}\text { Proj. } \\
\text { 2nd rev. }\end{array}$ & $\begin{array}{l}\text { Proj. } \\
\text { 3rd rev. }\end{array}$ & & & & & \\
\hline & \multicolumn{12}{|c|}{ (Annual percentage changes, unless otherwise indicated) } \\
\hline \multicolumn{13}{|l|}{ National income and prices } \\
\hline Nominal GDP (millions of dalasis) & 12,042 & 13,182 & 14,248 & 16,007 & 16,007 & 17,900 & 17,901 & 20,019 & 22,228 & 24,622 & 27,080 & 29,712 \\
\hline Nominal GDP & 20.1 & 9.5 & 8.1 & 12.3 & 12.3 & 11.8 & 11.8 & 11.8 & 11.0 & 10.8 & 10.0 & 9.7 \\
\hline GDP at constant prices & 7.0 & 5.1 & 6.5 & 7.0 & 6.3 & 6.5 & 5.5 & 6.0 & 6.0 & 6.0 & 5.5 & 5.5 \\
\hline Consumer price index (period average) & 14.3 & 5.0 & 2.1 & 5.0 & 5.4 & 5.0 & 6.0 & 5.5 & 4.8 & 4.5 & 4.3 & 4.0 \\
\hline Consumer price index (end of period) & 8.1 & 4.8 & 0.4 & 5.0 & 6.0 & 5.0 & 6.0 & 5.0 & 4.5 & 4.5 & 4.0 & 4.0 \\
\hline External sector & & & & & & & & & & & & \\
\hline Exports, f.o.b. ${ }^{1}$ & 10.5 & -16.5 & 3.9 & 4.5 & 8.8 & 3.9 & 11.0 & 4.5 & 6.2 & 5.7 & 5.1 & 4.5 \\
\hline Of which: domestic exports & 47.5 & -72.4 & 143.9 & 17.3 & -27.9 & 3.5 & 0.9 & 2.7 & 12.0 & 7.2 & 7.2 & 3.5 \\
\hline Imports, f.o.b. ${ }^{1}$ & 46.2 & 10.2 & -0.6 & 21.5 & 18.4 & 17.8 & 20.7 & 6.7 & 7.3 & 7.4 & 6.8 & 6.7 \\
\hline Terms of trade ${ }^{2}$ & -10.2 & -13.8 & -3.0 & -0.3 & -6.1 & 0.7 & -16.2 & -0.8 & 1.7 & 1.2 & 1.1 & 1.0 \\
\hline Nominal effective exchange rate (period average) & -11.3 & 4.2 & 0.5 & $\ldots$ & 7.6 & $\ldots$ & $\ldots$ & $\ldots$ & $\ldots$ & $\ldots$ & $\ldots$ & $\ldots$ \\
\hline Real effective exchange rate (period average) & -1.2 & 6.3 & -0.3 & $\ldots$ & 10.7 & $\ldots$ & $\ldots$ & $\ldots$ & $\ldots$ & $\ldots$ & $\ldots$ & $\ldots$ \\
\hline Money and credit & & & & (Percent & change; i & $\mathrm{n}$ beginnin & g-of-yea & $\mathrm{r}$ broad $\mathrm{m}$ & oney) & & & \\
\hline Broad money & 18.3 & 13.1 & 26.2 & 8.6 & 6.7 & 11.8 & 12.2 & 12.1 & 11.3 & 10.9 & 10.4 & 10.0 \\
\hline Net foreign assets & 28.8 & 0.3 & 17.8 & -3.6 & -3.5 & 4.1 & 4.7 & 2.9 & 4.5 & 3.1 & 3.9 & 3.8 \\
\hline Net domestic assets & -10.5 & 12.8 & 8.5 & 12.2 & 10.1 & 7.7 & 7.5 & 9.2 & 6.8 & 7.9 & 6.5 & 6.2 \\
\hline Credit to the government (net) ${ }^{3}$ & -10.6 & 6.3 & 3.8 & -3.8 & -5.0 & -2.1 & 4.8 & -0.4 & -1.6 & -1.7 & -1.0 & -0.3 \\
\hline Credit to the private sector and public enterprises & -6.5 & 5.5 & 8.4 & 2.8 & 4.3 & 3.7 & 4.5 & 4.5 & 4.6 & 4.6 & 4.7 & 4.8 \\
\hline Claims on foreign exchange bureaus & -1.2 & 0.0 & 0.0 & 0.0 & 0.0 & 0.0 & 0.0 & 0.0 & 0.0 & 0.0 & 0.0 & 0.0 \\
\hline Other items net & 7.7 & 1.0 & -3.7 & 13.2 & 10.9 & 6.1 & -1.8 & 5.0 & 3.8 & 5.0 & 2.8 & 1.8 \\
\hline Velocity (GDP/average broad money) & 2.4 & 2.3 & 1.9 & 2.0 & 2.0 & 2.0 & 2.0 & 2.0 & 2.0 & 2.0 & 2.0 & 2.0 \\
\hline Yield on treasury bills (percent per year) ${ }^{4}$ & 28.0 & 12.5 & 10.4 & 11.6 & 11.9 & $\ldots$ & 11.7 & $\ldots$ & $\ldots$ & $\ldots$ & $\ldots$ & $\ldots$ \\
\hline & & & & & & Percent of & GDP) & & & & & \\
\hline Savings and investment & & & & & & & & & & & & \\
\hline Gross investment & 30.0 & 26.8 & 28.4 & 23.6 & 23.2 & 24.9 & 25.2 & 25.3 & 25.9 & 24.5 & 24.0 & 23.6 \\
\hline Gross domestic savings & 9.3 & 4.6 & 11.9 & 5.3 & 7.6 & 5.0 & 6.8 & 7.4 & 8.5 & 8.0 & 8.2 & 8.0 \\
\hline Gross national savings & 23.9 & 11.7 & 16.9 & 12.8 & 10.8 & 12.8 & 10.8 & 12.2 & 13.7 & 12.7 & 12.8 & 12.4 \\
\hline Central government budget & & & & & & & & & & & & \\
\hline Domestic revenue & 20.9 & 19.7 & 21.2 & 22.1 & 21.7 & 21.1 & 21.1 & 21.1 & 21.1 & 21.0 & 21.0 & 21.0 \\
\hline Grants & 4.5 & 1.7 & 1.3 & 3.8 & 1.2 & 3.9 & 2.0 & 3.1 & 3.7 & 3.5 & 3.3 & 3.3 \\
\hline Total expenditure and net lending & 31.1 & 30.0 & 29.6 & 25.7 & 22.7 & 29.1 & 26.2 & 28.2 & 28.4 & 26.7 & 26.4 & 26.3 \\
\hline Overall balance, including grants & -6.2 & -9.2 & -7.8 & -0.6 & 0.5 & -4.1 & -3.0 & -4.0 & -3.6 & -2.3 & -2.1 & -2.0 \\
\hline Basic balance ${ }^{5}$ & 2.4 & -0.1 & 1.4 & 4.1 & 3.8 & 1.4 & 1.4 & 1.5 & 2.0 & 2.2 & 1.8 & 1.2 \\
\hline Basic primary balance $^{6}$ & 9.6 & 8.5 & 8.6 & 9.0 & 8.9 & 4.9 & 5.0 & 4.3 & 4.3 & 4.3 & 3.6 & 2.8 \\
\hline Net foreign financing & 5.7 & 5.5 & 6.1 & 0.8 & 1.0 & 4.6 & 3.5 & 4.5 & 4.7 & 3.5 & 2.8 & 2.3 \\
\hline Net domestic financing & 0.5 & 3.7 & 1.7 & -0.2 & -1.5 & -0.5 & -0.5 & -0.6 & -1.1 & -1.2 & -0.7 & -0.2 \\
\hline Stock of domestic public debt & 32.9 & 35.5 & 32.2 & 30.5 & 28.4 & 24.3 & 22.6 & 19.2 & 15.9 & 12.8 & 10.9 & 9.6 \\
\hline External sector & & & & & & & & & & & & \\
\hline Current account balance & & & & & & & & & & & & \\
\hline Excluding official transfers & -14.7 & -20.2 & -14.7 & -16.1 & -13.7 & -17.5 & -16.4 & -16.2 & -15.9 & -15.3 & -14.6 & -14.4 \\
\hline Including official transfers & -6.1 & -15.1 & -11.5 & -10.8 & -12.5 & -12.2 & -14.3 & -13.1 & -12.2 & -11.8 & -11.3 & -11.1 \\
\hline & & & & (Millions & of U.S. do & ollars, unle & ss other & wise indic & ated) & & & \\
\hline Current account balance & & & & & & & & & & & & \\
\hline Excluding official transfers & -58.8 & -93.3 & -74.6 & -104.1 & -88.1 & -138.7 & -127.6 & -136.5 & -145.5 & -151.4 & -156.0 & -166.4 \\
\hline Including official transfers & -24.6 & -69.6 & -58.3 & -70.1 & -80.3 & -96.7 & -111.8 & -110.3 & -111.3 & -117.0 & -120.4 & -128.4 \\
\hline Overall balance of payments & 31.3 & 13.0 & 24.4 & 10.6 & 31.9 & -3.1 & -1.1 & -5.1 & 4.6 & -0.4 & 4.8 & 5.7 \\
\hline Gross official reserves & 84.0 & 96.6 & 118.6 & 120.2 & 141.5 & 130.0 & 153.0 & 160.0 & 173.0 & 177.0 & 186.0 & 194.0 \\
\hline In months of imports, c.i.f. & 4.3 & 4.5 & 5.5 & 4.8 & 5.5 & 4.9 & 4.9 & 4.8 & 4.9 & 4.7 & 4.6 & 4.5 \\
\hline External public debt & & & & & & & & & & & & \\
\hline Stock $^{7}$ & 580.7 & 628.2 & 676.7 & 323.4 & 299.4 & 377.0 & 325.9 & 363.3 & 404.7 & 438.8 & 467.9 & 490.8 \\
\hline Stock (percent of GDP) & 146.5 & 134.7 & 133.6 & 49.9 & 46.5 & 47.5 & 41.8 & 43.1 & 44.3 & 44.3 & 43.8 & 0.0 \\
\hline Net present value of debt (percent of exports) ${ }^{8}$ & $\ldots$ & 215.4 & 101.9 & 106.8 & 113.9 & 118.5 & 117.6 & 126.4 & 133.3 & 136.8 & 138.0 & 137.2 \\
\hline External debt service (percent of exports) $)^{8}$ & 17.7 & 17.7 & 16.5 & 18.5 & 19.5 & 7.0 & 8.0 & 7.8 & 7.2 & 8.2 & 8.5 & 9.5 \\
\hline Use of Fund resources & & & & & & Millions of $S$ & SDRs) & & & & & \\
\hline Purchases/disbursements & 0.0 & 0.0 & 0.0 & 4.0 & 4.0 & 4.0 & 4.0 & 4.0 & 2.0 & 0.0 & 0.0 & 0.0 \\
\hline Repurchases/repayments & -7.6 & -1.4 & -2.7 & -2.8 & -11.8 & 0.0 & 0.0 & 0.0 & 0.0 & 0.0 & -0.2 & -1.0 \\
\hline Credit outstanding & 15.9 & 14.6 & 11.8 & $\ldots$ & & 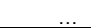 & $\ldots$ & $\ldots$ & $\ldots$ & $\ldots$ & & \\
\hline
\end{tabular}

Sources: Gambian authorities; and IMF staff estimates and projections.

${ }^{1}$ Computed based on values in U.S. dollars.

${ }^{2}$ Excluding reexports and imports for reexport.

${ }^{3}$ Including advances to the government in foreign currencies.

${ }^{4}$ Weighted average for all maturities based on weekly auction data for the month of December; and for 2008, data are for June 2008.

${ }^{5}$ Defined as domestic revenue minus expenditure and net lending, excluding externally financed capital expenditure.

${ }^{6}$ Defined as domestic revenue minus expenditure and net lending, excluding interest payments and externally financed capital expenditure.

${ }^{7}$ Reflects HIPC and MDRI debt relief delivered at end-2007.

${ }^{8}$ Exports of goods and nonfactor services (not including reexports). 
Table 2. The Gambia: Central Government Operations, 2004-13 (Millions of dalasis, unless otherwise indicated)

\begin{tabular}{|c|c|c|c|c|c|c|c|c|c|c|c|c|}
\hline & \multirow{2}{*}{\begin{tabular}{|c|}
2004 \\
Outturn \\
\end{tabular}} & \multirow{2}{*}{$\begin{array}{c}2005 \\
\text { Outturn }\end{array}$} & \multirow{2}{*}{\begin{tabular}{c|}
2006 \\
Outturn \\
\end{tabular}} & \multicolumn{2}{|c|}{2007} & \multicolumn{2}{|c|}{2008} & \multirow{2}{*}{$\begin{array}{c}2009 \\
3 \text { rd rev. }\end{array}$} & \multirow{2}{*}{$\begin{array}{c}2010 \\
3 \mathrm{rd} \mathrm{rev} \\
\end{array}$} & \multirow{2}{*}{$\begin{array}{c}2011 \\
3 \text { rd rev. }\end{array}$} & \multirow{2}{*}{$\begin{array}{c}2012 \\
3 \text { rd rev. }\end{array}$} & \multirow{2}{*}{$\begin{array}{r}2013 \\
\text { 3rd rev }\end{array}$} \\
\hline & & & & 2nd rev. & Outturn & 2nd rev. & 3rd rev. & & & & & \\
\hline Revenue and grants & 3,065 & 2,823 & 3,203 & 4,138 & 3,663 & 4,476 & 4,142 & 4,847 & 5,514 & 6,029 & 6,580 & 7,209 \\
\hline Revenue & 2,518 & 2,603 & 3,024 & 3,535 & 3,468 & 3,771 & 3,779 & 4,226 & 4,682 & 5,174 & 5,677 & 6,229 \\
\hline Tax revenue & 2,245 & 2,263 & 2,678 & 3,094 & 3,037 & 3,363 & 3,379 & 3,741 & 4,154 & 4,606 & 5,061 & 5,552 \\
\hline Nontax revenue & 273 & 340 & 346 & 441 & 431 & 408 & 400 & 485 & 527 & 568 & 616 & 667 \\
\hline Grants & 547 & 220 & 180 & 603 & 194 & 705 & 363 & 621 & 833 & 855 & 903 & 980 \\
\hline Of which: Projects & 396 & 203 & 163 & 554 & 174 & 669 & 328 & 428 & 815 & 838 & 885 & 980 \\
\hline Expenditure and net lending & 3,750 & 3,961 & 4,211 & 4,113 & 3,635 & 5,205 & 4,686 & 5,639 & 6,323 & 6,586 & 7,144 & 7,813 \\
\hline Current expenditure & 2,036 & 2,420 & 2,584 & 2,653 & 2,586 & 2,812 & 2,838 & 3,113 & 3,341 & 3,648 & 4,032 & 4,482 \\
\hline Wages and salaries & 518 & 554 & 653 & 724 & 680 & 918 & 918 & 1,193 & 1,324 & 1,467 & 1,614 & 1,783 \\
\hline Other charges & 584 & 736 & 1,010 & 1,147 & 1,091 & 1,143 & 1,151 & 1,221 & 1,356 & 1,498 & 1,755 & 2,030 \\
\hline Interest & 868 & 1,131 & 921 & 782 & 815 & 622 & 640 & 568 & 523 & 510 & 485 & 473 \\
\hline External $^{1}$ & 235 & 241 & 232 & 197 & 231 & 72 & 72 & 83 & 90 & 105 & 116 & 127 \\
\hline Domestic & 633 & 890 & 689 & 585 & 584 & 550 & 568 & 485 & 433 & 405 & 369 & 345 \\
\hline HIPC and MDRI financed & 67 & 0 & 0 & 0 & 0 & 129 & 129 & 131 & 138 & 173 & 178 & 197 \\
\hline Capital expenditure and net lending & 1,710 & 1,419 & 1,627 & 1,461 & 1,048 & 2,333 & 1,788 & 2,446 & 2,894 & 2,840 & 3,003 & 3,212 \\
\hline Capital expenditure & 1,734 & 1,450 & 1,564 & 1,389 & 973 & 2,223 & 1,679 & 2,315 & 2,738 & 2,752 & 2,935 & 3,144 \\
\hline Externally financed & 1,517 & 1,341 & 1,487 & 1,238 & 780 & 1,694 & 1,153 & 1,706 & 2,076 & 1,963 & 1,945 & 1,942 \\
\hline GLF (Gambia Local Fund) & 89 & 106 & 77 & 151 & 192 & 336 & 332 & 389 & 455 & 616 & 812 & 1,040 \\
\hline HIPC and MDRI financed & 128 & 2 & 0 & 0 & 0 & 194 & 194 & 220 & 207 & 173 & 178 & 161 \\
\hline Net lending & -23 & -30 & 63 & 72 & 76 & 110 & 110 & 131 & 156 & 88 & 68 & 68 \\
\hline Contingency & 0 & 0 & 0 & 0 & 0 & 60 & 60 & 80 & 89 & 98 & 108 & 119 \\
\hline Extrabudgetary expenditure & 5 & 121 & 0 & 0 & 0 & 0 & 0 & 0 & 0 & 0 & 0 & 0 \\
\hline Overall balance & -685 & $-1,138$ & $-1,008$ & 25 & 28 & -730 & -544 & -792 & -809 & -557 & -565 & -604 \\
\hline Statistical discrepancy ${ }^{2}$ & -63 & -72 & -104 & -127 & 48 & 0 & 0 & 0 & 0 & 0 & 0 & 0 \\
\hline Adjusted overall balance & -748 & $-1,210$ & $-1,112$ & -102 & 76 & -730 & -544 & -792 & -809 & -557 & -565 & -604 \\
\hline Financing & 748 & 1,210 & 1,112 & 102 & -76 & 730 & 544 & 792 & 809 & 557 & 565 & 604 \\
\hline External (net) & 690 & 728 & 876 & 129 & 158 & 826 & 627 & 903 & 1,053 & 857 & 752 & 678 \\
\hline Borrowing & 1,121 & 1,138 & 1,308 & 634 & 586 & 1,024 & 825 & 1,112 & 1,261 & 1,125 & 1,060 & 962 \\
\hline Amortization $^{1}$ & -479 & -456 & -477 & -506 & -428 & -198 & -198 & -209 & -208 & -268 & -308 & -387 \\
\hline Domestic & 58 & 482 & 236 & -27 & -234 & -96 & -83 & -111 & -245 & -300 & -188 & -74 \\
\hline Net borrowing & 58 & 433 & 334 & 9 & -428 & -318 & -135 & -111 & -245 & -300 & -188 & -74 \\
\hline Bank & -578 & 340 & 231 & 119 & -68 & -174 & -56 & -36 & -163 & -200 & -125 & -50 \\
\hline Of which: MDRI account & & 0 & 0 & 0 & 0 & 40 & 80 & 80 & 80 & 80 & 15 & 0 \\
\hline Nonbank & 636 & 93 & 103 & 59 & -267 & -87 & 12 & 17 & -5 & -66 & -63 & -25 \\
\hline Repayment of domestic debt & 0 & 0 & 0 & -169 & -93 & -57 & -92 & -92 & -77 & -34 & 0 & 0 \\
\hline Capital revenue & -- & 0 & 110 & 21 & 24 & 15 & 15 & 0 & 0 & 0 & 0 & 0 \\
\hline Change in arrears (- decrease) & -- & -5 & -209 & -425 & -456 & -184 & -106 & 0 & 0 & 0 & 0 & 0 \\
\hline Privatization proceeds & 0 & 54 & 0 & 368 & 626 & 390 & 143 & 0 & 0 & 0 & 0 & 0 \\
\hline \multicolumn{13}{|l|}{ Memorandum items: } \\
\hline Basic balance $^{3}$ & 284 & -16 & 196 & 659 & 614 & 259 & 246 & 293 & 434 & 551 & 477 & 358 \\
\hline Basic primary balance ${ }^{4}$ & 1,152 & 1,114 & 1,221 & 1,441 & 1,429 & 882 & 886 & 861 & 957 & 1,062 & 963 & 831 \\
\hline Gross domestic interest-bearing debt & 3,957 & 4,675 & 4,582 & 4,889 & 4,546 & 4,349 & 4,039 & 3,848 & 3,523 & 3,143 & 2,940 & 2,855 \\
\hline Stock of arrears & & 770 & 561 & 136 & 106 & 0 & 0 & 0 & 0 & 0 & 0 & 0 \\
\hline \multicolumn{13}{|l|}{ Stock of HIPC and MDRI debt relief } \\
\hline Of which: IMF & & & & & 336 & & & & & & & \\
\hline IDA & & & & & 4,652 & & & & & & & \\
\hline AfDF & & & & & 3,518 & & & & & & & \\
\hline Resources freed by MDRI debt relief & & & & 106 & 108 & 502 & 515 & 521 & 531 & 345 & 357 & 358 \\
\hline Amortization & & & & 99 & 101 & 380 & 390 & 408 & 424 & 0 & 0 & 0 \\
\hline Interest payments & & & & 10 & 11 & 117 & 120 & 113 & 106 & 0 & 0 & 0 \\
\hline Uses of resources freed by MDRI debt & & & & 106 & 108 & 502 & 515 & 521 & 531 & 345 & 357 & 358 \\
\hline Current expenditures & & & & 0 & 0 & 129 & 129 & 131 & 138 & 173 & 178 & 197 \\
\hline Capital expenditures & & & & 0 & 0 & 194 & 194 & 220 & 207 & 173 & 178 & 161 \\
\hline Savings & & & & 106 & 108 & 179 & 193 & 170 & 186 & 0 & 0 & 0 \\
\hline Exp. financed by privatization proceeds & & & & 70 & 159 & 301 & 390 & 221 & 0 & 0 & 0 & 0 \\
\hline
\end{tabular}

Sources: Gambian authorities; IMF staff estimates and projections.

${ }^{1}$ After MDRI debt relief from 2007 onward.

${ }^{2}$ The difference between financing and the overall balance of revenue and expenditure.

${ }^{3}$ Domestic revenue - expenditure and net lending, excluding externally financed capital spending

${ }^{4}$ Domestic revenue - expenditure and net lending, excluding interest payments and externally financed capital spending. 
Table 2. The Gambia: Central Government Operations, 2004-13 (Percent of GDP

\begin{tabular}{|c|c|c|c|c|c|c|c|c|c|c|c|c|}
\hline \multicolumn{13}{|c|}{ (Percent of GUP) } \\
\hline & \multirow{2}{*}{$\begin{array}{c}2004 \\
\text { Actual } \\
\end{array}$} & \multirow{2}{*}{$\frac{2005}{\text { Outturn }}$} & \multirow{2}{*}{$\frac{2006}{\text { Outturn }}$} & \multicolumn{2}{|c|}{2007} & \multicolumn{2}{|c|}{2008} & \multirow{2}{*}{$\begin{array}{c}2009 \\
\text { 3rd rev. }\end{array}$} & \multirow{2}{*}{$\begin{array}{c}2010 \\
\text { 3rd rev. }\end{array}$} & \multirow{2}{*}{$\begin{array}{c}2011 \\
\text { 3rd rev. }\end{array}$} & \multirow{2}{*}{$\frac{2012}{3 \text { rd rev. }}$} & \multirow{2}{*}{$\begin{array}{c}2013 \\
\text { 3rd rev. }\end{array}$} \\
\hline & & & & 2nd rev. & Outturn & 2nd rev. & 3rd rev. & & & & & \\
\hline Revenue and grants & 25.5 & 21.4 & 22.5 & 25.9 & 22.9 & 25.0 & 23.1 & 24.2 & 24.8 & 24.5 & 24.3 & 24.3 \\
\hline Revenue & 20.9 & 19.7 & 21.2 & 22.1 & 21.7 & 21.1 & 21.1 & 21.1 & 21.1 & 21.0 & 21.0 & 21.0 \\
\hline Tax revenue & 18.6 & 17.2 & 18.8 & 19.3 & 19.0 & 18.8 & 18.9 & 18.7 & 18.7 & 18.7 & 18.7 & 18.7 \\
\hline Nontax revenue & 2.3 & 2.3 & 2.4 & 2.8 & 2.7 & 2.3 & 2.2 & 2.4 & 2.4 & 2.3 & 2.3 & 2.2 \\
\hline Grants & 4.5 & 1.7 & 1.3 & 3.8 & 1.2 & 3.9 & 2.0 & 3.1 & 3.7 & 3.5 & 3.3 & 3.3 \\
\hline Of which: Projects & 3.3 & 1.5 & 1.1 & 3.5 & 1.1 & 3.7 & 1.8 & 2.1 & 3.7 & 3.4 & 3.3 & 3.3 \\
\hline Expenditure and net lending & 31.1 & 30.0 & 29.6 & 25.7 & 22.7 & 29.1 & 26.2 & 28.2 & 28.4 & 26.7 & 26.4 & 26.3 \\
\hline Current expenditure & 16.9 & 18.4 & 18.1 & 16.6 & 16.2 & 15.7 & 15.9 & 15.6 & 15.0 & 14.8 & 14.9 & 15.1 \\
\hline Wages and salaries & 4.3 & 4.2 & 4.6 & 4.5 & 4.3 & 5.1 & 5.1 & 6.0 & 6.0 & 6.0 & 6.0 & 6.0 \\
\hline Other charges & 4.8 & 5.6 & 7.1 & 7.2 & 6.8 & 6.4 & 6.4 & 6.1 & 6.1 & 6.1 & 6.5 & 6.8 \\
\hline Interest & 7.2 & 8.6 & 6.5 & 4.9 & 5.1 & 3.5 & 3.6 & 2.8 & 2.4 & 2.1 & 1.8 & 1.6 \\
\hline External' & 1.9 & 1.8 & 1.6 & 1.2 & 1.4 & 0.4 & 0.4 & 0.4 & 0.4 & 0.4 & 0.4 & 0.4 \\
\hline Domestic & 5.3 & 6.8 & 4.8 & 3.7 & 3.6 & 3.1 & 3.2 & 2.4 & 1.9 & 1.6 & 1.4 & 1.2 \\
\hline HIPC and MDRI financed & 0.6 & 0.0 & 0.0 & 0.0 & 0.0 & 0.7 & 0.7 & 0.7 & 0.6 & 0.7 & 0.7 & 0.7 \\
\hline Capital expenditure and net lending & 14.2 & 10.8 & 11.4 & 9.1 & 6.5 & 13.0 & 10.0 & 12.2 & 13.0 & 11.5 & 11.1 & 10.8 \\
\hline Capital expenditure & 14.4 & 11.0 & 11.0 & 8.7 & 6.1 & 12.4 & 9.4 & 11.6 & 12.3 & 11.2 & 10.8 & 10.6 \\
\hline Externally financed & 12.6 & 10.2 & 10.4 & 7.7 & 4.9 & 9.5 & 6.4 & 8.5 & 9.3 & 8.0 & 7.2 & 6.5 \\
\hline GLF (Gambia Local Fund) & 0.7 & 0.8 & 0.5 & 0.9 & 1.2 & 1.9 & 1.9 & 1.9 & 2.0 & 2.5 & 3.0 & 3.5 \\
\hline HIPC and MDRI financed & 1.1 & 0.0 & 0.0 & 0.0 & 0.0 & 1.1 & 1.1 & 1.1 & 0.9 & 0.7 & 0.7 & 0.5 \\
\hline Net lending & -0.2 & -0.2 & 0.4 & 0.4 & 0.5 & 0.6 & 0.6 & 0.7 & 0.7 & 0.4 & 0.3 & 0.2 \\
\hline Contingency & 0.0 & 0.0 & 0.0 & 0.0 & 0.0 & 0.3 & 0.3 & 0.4 & 0.4 & 0.4 & 0.4 & 0.4 \\
\hline Extrabudgetary expenditure & 0.0 & 0.9 & 0.0 & 0.0 & 0.0 & 0.0 & 0.0 & 0.0 & 0.0 & 0.0 & 0.0 & 0.0 \\
\hline Overall balance & -5.7 & -8.6 & -7.1 & 0.2 & 0.2 & -4.1 & -3.0 & -4.0 & -3.6 & -2.3 & -2.1 & -2.0 \\
\hline Statistical discrepancy ${ }^{<}$ & -0.5 & -0.5 & -0.7 & -0.8 & 0.3 & 0.0 & 0.0 & 0.0 & 0.0 & 0.0 & 0.0 & 0.0 \\
\hline Adjusted overall balance & -6.2 & -9.2 & -7.8 & -0.6 & 0.5 & -4.1 & -3.0 & -4.0 & -3.6 & -2.3 & -2.1 & -2.0 \\
\hline Financing & 6.2 & 9.2 & 7.8 & 0.6 & -0.5 & 4.1 & 3.0 & 4.0 & 3.6 & 2.3 & 2.1 & 2.0 \\
\hline External (net) & 5.7 & 5.5 & 6.1 & 0.8 & 1.0 & 4.6 & 3.5 & 4.5 & 4.7 & 3.5 & 2.8 & 2.3 \\
\hline Borrowing & 9.3 & 8.6 & 9.2 & 4.0 & 3.7 & 5.7 & 4.6 & 5.6 & 5.7 & 4.6 & 3.9 & 3.2 \\
\hline Amortization ${ }^{1}$ & -4.0 & -3.5 & -3.3 & -3.2 & -2.7 & -1.1 & -1.1 & -1.0 & -0.9 & -1.1 & -1.1 & -1.3 \\
\hline Domestic & 0.5 & 3.7 & 1.7 & -0.2 & -1.5 & -0.5 & -0.5 & -0.6 & -1.1 & -1.2 & -0.7 & -0.2 \\
\hline Net borrowing & 0.5 & 3.3 & 2.3 & 0.1 & -2.7 & -1.8 & -0.8 & -0.6 & -1.1 & -1.2 & -0.7 & -0.2 \\
\hline Bank & -4.8 & 2.6 & 1.6 & 0.7 & -0.4 & -1.0 & -0.3 & -0.2 & -0.7 & -0.8 & -0.5 & -0.2 \\
\hline Of which: MDRI account & & & & 0.0 & 0.0 & 0.2 & 0.4 & 0.4 & 0.4 & 0.3 & 0.0 & 0.0 \\
\hline Nonbank & 5.3 & 0.7 & 0.7 & 0.4 & -1.7 & -0.5 & 0.1 & 0.1 & 0.0 & -0.3 & -0.2 & -0.1 \\
\hline Repayment of domestic debt & 0.0 & 0.0 & 0.0 & -1.1 & -0.6 & -0.3 & -0.5 & -0.5 & -0.3 & -0.1 & 0.0 & 0.0 \\
\hline Capital revenue & 0.0 & 0.0 & 0.8 & 0.1 & 0.1 & 0.1 & 0.1 & 0.0 & 0.0 & 0.0 & 0.0 & 0.0 \\
\hline Change in arrears (- decrease) & 0.0 & 0.0 & -1.5 & -2.7 & -2.8 & -1.0 & -0.6 & 0.0 & 0.0 & 0.0 & 0.0 & 0.0 \\
\hline Privatization proceeds & 0.0 & 0.4 & 0.0 & 2.3 & 3.9 & 2.2 & 0.8 & 0.0 & 0.0 & 0.0 & 0.0 & 0.0 \\
\hline \multicolumn{13}{|l|}{ Memorandum items: } \\
\hline Basic balance $^{s}$ & 2.4 & -0.1 & 1.4 & 4.1 & 3.8 & 1.4 & 1.4 & 1.5 & 2.0 & 2.2 & 1.8 & 1.2 \\
\hline Basic primary balance $^{4}$ & 9.6 & 8.5 & 8.6 & 9.0 & 8.9 & 4.9 & 5.0 & 4.3 & 4.3 & 4.3 & 3.6 & 2.8 \\
\hline Gross domestic interest-bearing debt & 32.9 & 35.5 & 32.2 & 30.5 & 28.4 & 24.3 & 22.6 & 19.2 & 15.9 & 12.8 & 10.9 & 9.6 \\
\hline Stock of arrears & & 5.8 & 3.9 & 0.9 & 0.7 & 0.0 & 0.0 & 0.0 & 0.0 & 0.0 & 0.0 & 0.0 \\
\hline \multicolumn{13}{|l|}{ Stock of HIPC and MDRI debt relief } \\
\hline Of which: IMF & & & & & 2.1 & & & & & & & \\
\hline IDA & & & & & 29.1 & & & & & & & \\
\hline AfDF & & & & & 22.0 & & & & & & & \\
\hline Resources freed by MDRI debt relief & & & & 0.7 & 0.7 & 2.8 & 2.9 & 2.6 & 2.4 & 1.4 & 1.3 & 1.2 \\
\hline Amortization & & & & 0.6 & 0.6 & 2.1 & 2.2 & 2.0 & 1.9 & 0.0 & 0.0 & 0.0 \\
\hline Interest payments & & & & 0.1 & 0.1 & 0.7 & 0.7 & 0.6 & 0.5 & 0.0 & 0.0 & 0.0 \\
\hline \multicolumn{4}{|c|}{ Uses of resources freed by MDRI debt relief } & 0.7 & 0.7 & 2.8 & 2.9 & 2.6 & 2.4 & 0.0 & 1.3 & 0.0 \\
\hline Current expenditures & & & & 0.0 & 0.0 & 0.7 & 0.7 & 0.7 & 0.6 & 0.0 & 0.7 & 0.0 \\
\hline Capital expenditures & & & & 0.0 & 0.0 & 1.1 & 1.1 & 1.1 & 0.9 & 0.0 & 0.6 & 0.0 \\
\hline Savings & & & & 0.7 & 0.7 & 1.0 & 1.1 & 0.8 & 0.8 & 0.0 & 0.0 & 0.0 \\
\hline Exp. financed by privatization proceed & & & & 0.4 & 1.0 & 1.7 & 2.2 & 1.1 & 0.0 & 0.0 & 0.0 & 0.0 \\
\hline
\end{tabular}

Sources: Gambian authorities; IMF staff estimates and projections.

'After MDRI debt relief from 2007 onward.

${ }^{<}$The difference between financing and the overall balance of revenue and expenditure.

${ }^{3}$ Domestic revenue - expenditure and net lending, excluding externally financed capital spending.

${ }^{4}$ Domestic revenue - expenditure and net lending, excluding interest payments and externally financed capital spending. 
Table 3. The Gambia: Monetary Survey, 2004-13

\begin{tabular}{|c|c|c|c|c|c|c|c|c|c|c|c|}
\hline & \multirow{2}{*}{$\begin{array}{l}2004 \\
\text { Dec. }\end{array}$} & \multirow{2}{*}{$\begin{array}{l}2005 \\
\text { Dec. }\end{array}$} & \multirow{2}{*}{$\begin{array}{l}2006 \\
\text { Dec. } \\
\text { Act. }\end{array}$} & \multicolumn{2}{|c|}{2007} & \multirow{2}{*}{$\begin{array}{c}2008 \\
\text { Dec. } \\
\text { Proj. } \\
\text { 3rd. rev. }\end{array}$} & \multirow{2}{*}{$\begin{array}{c}2009 \\
\text { Dec. } \\
\text { Proj. } \\
\text { 3rd rev. }\end{array}$} & \multirow{2}{*}{$\begin{array}{c}2010 \\
\text { Dec. } \\
\text { Proj. } \\
\text { 3rd rev. }\end{array}$} & \multirow{2}{*}{$\begin{array}{c}2011 \\
\text { Dec. } \\
\text { Proj. } \\
\text { 3rd rev. }\end{array}$} & \multirow{2}{*}{$\begin{array}{c}2012 \\
\text { Dec. } \\
\text { Proj. } \\
\text { 3rd rev. }\end{array}$} & \multirow{2}{*}{$\begin{array}{c}2013 \\
\text { Dec. } \\
\text { Proj. } \\
\text { 3rd rev. }\end{array}$} \\
\hline & & & & $\begin{array}{c}\text { Dec. } \\
\text { Proj. } \\
\text { 2nd rev. }\end{array}$ & $\begin{array}{l}\text { Dec. } \\
\text { Act. }\end{array}$ & & & & & & \\
\hline & \multicolumn{11}{|c|}{ (Millions of dalasis, unless otherwise indicated; end of period) } \\
\hline Net foreign assets & 3,184 & 3,201 & 4,292 & 4,010 & 4,022 & 4,409 & 4,679 & 5,148 & 5,504 & 6,000 & 6,534 \\
\hline Net domestic assets & 2,247 & 2,944 & 3,466 & 4,415 & 4,252 & 4,871 & 5,721 & 6,429 & 7,340 & 8,178 & 9,063 \\
\hline Domestic credit & 2,870 & 3,510 & 4,259 & 4,187 & 4,200 & 4,972 & 5,357 & 5,667 & 6,001 & 6,479 & 7,107 \\
\hline Claims on government (net) & 1,009 & 1,348 & 1,579 & 1,287 & 1,189 & 1,587 & 1,551 & 1,388 & 1,188 & 1,063 & 1,014 \\
\hline Claims on government (net) $)^{1}$ & 434 & 774 & 1,504 & 1,287 & 1,189 & 1,587 & 1,551 & 1,388 & 1,188 & 1,063 & 1,014 \\
\hline Advances to the government in foreign currencies ${ }^{2}$ & 575 & 575 & 75 & 0 & 0 & 0 & 0 & 0 & 0 & 0 & 0 \\
\hline Claims on the private sector and public enterprises ${ }^{3}$ & 1,677 & 1,979 & 2,497 & 2,716 & 2,829 & 3,201 & 3,622 & 4,095 & 4,629 & 5,232 & 5,910 \\
\hline Claims on public enterprises & 223 & 260 & 268 & 270 & 229 & 251 & 264 & 277 & 291 & 306 & 321 \\
\hline Claims on private sector & 1,454 & 1,719 & 2,230 & 2,446 & 2,600 & 2,950 & 3,358 & 3,818 & 4,338 & 4,927 & 5,589 \\
\hline Claims on foreign exchange bureaus ${ }^{4}$ & 183 & 183 & 183 & 183 & 183 & 183 & 183 & 183 & 183 & 183 & 183 \\
\hline Other items (net) & -622 & -566 & -793 & 229 & 51 & -101 & 364 & 761 & 1,339 & 1,700 & 1,956 \\
\hline Broad money & 5,432 & 6,145 & 7,758 & 8,425 & 8,274 & 9,280 & 10,399 & 11,577 & 12,844 & 14,178 & 15,597 \\
\hline Currency outside banks & 1,416 & 1,424 & 1,937 & 1,980 & 1,689 & 1,949 & 2,184 & 2,431 & 2,697 & 2,977 & 3,275 \\
\hline Deposits & 4,016 & 4,721 & 5,820 & 6,445 & 6,585 & 7,331 & 8,215 & 9,146 & 10,147 & 11,201 & 12,322 \\
\hline Contribution to growth of broad money & \multicolumn{11}{|c|}{ (Percent change in beginning-of-year broad money, unless otherwise indicated) } \\
\hline Broad money & 18.3 & 13.1 & 26.2 & 8.6 & 6.7 & 12.2 & 12.1 & 11.3 & 10.9 & 10.4 & 10.0 \\
\hline Net foreign assets & 28.8 & 0.3 & 17.8 & -3.6 & -3.5 & 4.7 & 2.9 & 4.5 & 3.1 & 3.9 & 3.8 \\
\hline Net domestic assets & -10.5 & 12.8 & 8.5 & 12.2 & 10.1 & 7.5 & 9.2 & 6.8 & 7.9 & 6.5 & 6.2 \\
\hline Domestic credit & -18.3 & 11.8 & 12.2 & -0.9 & -0.8 & 9.3 & 4.1 & 3.0 & 2.9 & 3.7 & 4.4 \\
\hline Claims on government (net) & -10.6 & 6.3 & 3.8 & -3.8 & -5.0 & 4.8 & -0.4 & -1.6 & -1.7 & -1.0 & -0.3 \\
\hline Claims on government (net) $)^{1}$ & -12.6 & 6.3 & 11.9 & -2.8 & -4.1 & 4.8 & -0.4 & -1.6 & -1.7 & -1.0 & -0.3 \\
\hline Advances to the government in foreign currencies ${ }^{2}$ & 2.0 & 0.0 & -8.1 & -1.0 & -1.0 & 0.0 & 0.0 & 0.0 & 0.0 & 0.0 & 0.0 \\
\hline Claims on the private sector and public enterprises ${ }^{3}$ & -6.5 & 5.5 & 8.4 & 2.8 & 4.3 & 4.5 & 4.5 & 4.6 & 4.6 & 4.7 & 4.8 \\
\hline Claims on public enterprises & -2.6 & 0.7 & 0.1 & 0.0 & -0.5 & 0.3 & 0.1 & 0.1 & 0.1 & 0.1 & 0.1 \\
\hline Claims on private sector & -3.9 & 4.9 & 8.3 & 2.8 & 4.8 & 4.2 & 4.4 & 4.4 & 4.5 & 4.6 & 4.7 \\
\hline Claims on foreign exchange bureaus ${ }^{4}$ & -1.2 & 0.0 & 0.0 & 0.0 & 0.0 & 0.0 & 0.0 & 0.0 & 0.0 & 0.0 & 0.0 \\
\hline Other items (net) & 7.7 & 1.0 & -3.7 & 13.2 & 10.9 & -1.8 & 5.0 & 3.8 & 5.0 & 2.8 & 1.8 \\
\hline \multicolumn{12}{|l|}{ Memorandum items: } \\
\hline \multicolumn{12}{|l|}{ Credit to the private sector and public enterprises } \\
\hline Twelve-month change (percent) & -15.1 & 18.0 & 26.2 & 8.8 & 13.3 & 13.2 & 13.1 & 13.1 & 13.0 & 13.0 & 13.0 \\
\hline Percent of GDP & 13.9 & 15.0 & 17.5 & 17.0 & 17.7 & 17.9 & 18.1 & 18.4 & 18.8 & 19.3 & 19.9 \\
\hline \multicolumn{12}{|l|}{ Selected ratios (in percent) } \\
\hline Currency outside banks/broad money & 26.1 & 23.2 & 25.0 & 23.5 & 20.4 & 21.0 & 21.0 & 21.0 & 21.0 & 21.0 & 21.0 \\
\hline Currency outside banks/deposits & 35.3 & 30.2 & 33.3 & 30.7 & 25.7 & 26.6 & 26.6 & 26.6 & 26.6 & 26.6 & 26.6 \\
\hline Deposits/broad money & 73.9 & 76.8 & 75.0 & 76.5 & 79.6 & 79.0 & 79.0 & 79.0 & 79.0 & 79.0 & 79.0 \\
\hline Nominal GDP (millions of dalasis) & 12,042 & 13,182 & 14,248 & 16,007 & 16,007 & 17,901 & 20,019 & 22,228 & 24,622 & 27,080 & 29,712 \\
\hline (percentage change) & 20.1 & 9.5 & 8.1 & 12.3 & 12.3 & 11.8 & 11.8 & 11.0 & 10.8 & 10.0 & 9.7 \\
\hline Velocity (calendar-year GDP/end-of-period broad money) & 2.2 & 2.1 & 1.8 & 1.9 & 1.9 & 1.9 & 1.9 & 1.9 & 1.9 & 1.9 & 1.9 \\
\hline Velocity (GDP/average broad money) & 2.4 & 2.3 & 1.9 & 2.0 & 2.0 & 2.0 & 2.0 & 2.0 & 2.0 & 2.0 & 2.0 \\
\hline Velocity (GDP/end-of-period broad money) & 2.2 & 2.1 & 1.8 & 1.9 & 1.9 & 1.9 & 1.9 & 1.9 & 1.9 & 1.9 & 1.9 \\
\hline Money multiplier (broad money/reserve money) & 2.64 & 2.66 & 2.71 & 2.76 & 3.01 & 3.05 & 3.05 & 3.05 & 3.05 & 3.05 & 3.05 \\
\hline
\end{tabular}

Sources: Gambian authorities; IMF staff estimates and projections.

Note: MDRI debt relief, incorporated for end-2007, has increased net foreign assets and decreased net domestic assets by D335 millions (at current exchange rate).

${ }^{1}$ Excluding advances to the government in foreign currencies.

${ }^{2}$ These advances reflect previously unrecorded public spending and borrowing in 2001, financed by the Central Bank of The Gambia (CBG), and the previously unrecorded

depletion of foreign exchange reserves in 2001-03 as reported by the authorities on October 28, 2003.

${ }^{3}$ In March 2003, the government instructed the CBG to lend the equivalent of D137 million in U.S. dollars to a newly created public enterprise for a seismic survey of offshore oil deposits.

${ }^{4}$ Claims on foreign exchange bureaus reflect the delayed delivery of foreign currency purchased on a spot basis. 
Table 4. The Gambia: Analytical Account of the Central Bank of The Gambia (CBG), 2004-13

(Millions of dalasis, unless otherwise indicated; end of period)

\begin{tabular}{|c|c|c|c|c|c|c|c|c|c|c|c|}
\hline & \multirow[t]{2}{*}{2004} & \multirow[t]{2}{*}{2005} & \multirow{2}{*}{$\begin{array}{c}2006 \\
\text { Dec. } \\
\text { Act. }\end{array}$} & \multicolumn{2}{|c|}{2007} & \multirow{2}{*}{$\begin{array}{c}2008 \\
\text { Dec. } \\
\text { Proj. } \\
\text { 3rd rev. }\end{array}$} & \multirow{2}{*}{$\begin{array}{c}2009 \\
\text { Dec. } \\
\text { Proj. } \\
\text { 3rd rev. }\end{array}$} & \multirow{2}{*}{$\begin{array}{c}2010 \\
\text { Dec. } \\
\text { Proj. } \\
\text { 3rd rev. }\end{array}$} & \multirow{2}{*}{$\begin{array}{c}2011 \\
\text { Dec. } \\
\text { Proj. } \\
\text { 3rd rev. }\end{array}$} & \multirow{2}{*}{$\begin{array}{c}2012 \\
\text { Dec. } \\
\text { Proj. } \\
\text { 3rd rev. }\end{array}$} & \multirow{2}{*}{$\begin{array}{c}2013 \\
\text { Dec. } \\
\text { Proj. } \\
\text { 3rd rev. }\end{array}$} \\
\hline & & & & $\begin{array}{c}\text { Dec. } \\
\text { Proj. } \\
\text { 2nd rev. }\end{array}$ & $\begin{array}{l}\text { Dec. } \\
\text { Act. }\end{array}$ & & & & & & \\
\hline Net foreign assets & $1,757.8$ & $2,132.3$ & $2,827.7$ & $2,509.3$ & $3,049.2$ & $3,298.0$ & $3,417.9$ & $3,730.3$ & $3,909.0$ & $4,213.0$ & $4,532.7$ \\
\hline Foreign assets & $2,492.5$ & $2,718.2$ & $3,326.8$ & $2,644.4$ & $3,191.6$ & $3,580.0$ & $3,855.3$ & $4,253.4$ & $4,446.6$ & $4,755.4$ & $5,047.7$ \\
\hline Foreign liabilities & -734.7 & -585.9 & -499.1 & -135.1 & -142.5 & -282.0 & -437.3 & -523.1 & -537.6 & -542.4 & -515.0 \\
\hline Net usable reserves (millions of U.S. dollars) & 55.4 & 71.1 & 94.9 & 107.2 & 126.9 & 131.3 & 132.2 & 142.1 & 146.0 & 155.2 & 164.6 \\
\hline Net domestic assets & 303.0 & 174.3 & 38.7 & 543.2 & -304.6 & -255.4 & -8.3 & 65.4 & 302.1 & 435.5 & 581.0 \\
\hline Domestic credit & 195.9 & -29.2 & -164.0 & -552.2 & -903.4 & -345.2 & -413.2 & -439.2 & -468.2 & -462.0 & -459.5 \\
\hline Claims on government (net) & -186.8 & -413.8 & -548.5 & -936.7 & $-1,294.0$ & -701.3 & -735.1 & -726.9 & -721.6 & -715.4 & -712.9 \\
\hline Claims on government (net) ${ }^{1}$ & -761.6 & -988.6 & -623.3 & -936.8 & $-1,294.0$ & -701.3 & -735.1 & -726.9 & -721.6 & -715.4 & -712.9 \\
\hline Advances to the government in foreign currencies ${ }^{2}$ & 574.8 & 574.8 & 74.7 & 0.0 & 0.0 & 0.0 & 0.0 & 0.0 & 0.0 & 0.0 & 0.0 \\
\hline Claims on banks (net) ${ }^{3}$ & 33.6 & 33.6 & 33.6 & 33.6 & 33.6 & 33.6 & 33.6 & 33.6 & 33.6 & 33.6 & 33.6 \\
\hline Claims on other resident sectors & 349.0 & 351.0 & 350.9 & 350.9 & 357.0 & 322.5 & 288.2 & 254.0 & 219.8 & 219.8 & 219.8 \\
\hline Claims on private sector & 28.8 & 30.8 & 30.7 & 30.7 & 36.8 & 36.5 & 36.5 & 36.5 & 36.5 & 36.5 & 36.5 \\
\hline Claims on public enterprises & 136.9 & 136.9 & 136.9 & 136.9 & 136.9 & 102.7 & 68.5 & 34.2 & 0.0 & 0.0 & 0.0 \\
\hline Claims on foreign exchange bureaus ${ }^{4}$ & 183.3 & 183.3 & 183.3 & 183.3 & 183.3 & 183.3 & 183.3 & 183.3 & 183.3 & 183.3 & 183.3 \\
\hline Other items (net) & 107.1 & 203.5 & 202.7 & $1,095.5$ & 598.8 & 89.7 & 404.9 & 504.7 & 770.3 & 897.5 & $1,040.5$ \\
\hline $\begin{array}{l}\text { Of which: Holdings of government's noninterest-bearing } \\
\text { securities }\end{array}$ & 199.0 & 199.0 & 534.9 & 534.9 & 415.4 & 873.4 & 774.6 & 700.2 & 616.8 & 543.5 & 464.6 \\
\hline Reserve money & $2,060.7$ & $2,306.6$ & $2,866.5$ & $3,052.5$ & $2,744.5$ & $3,042.6$ & $3,409.6$ & $3,795.7$ & $4,211.1$ & $4,648.5$ & $5,113.7$ \\
\hline Currency in circulation outside banks & $1,416.3$ & $1,424.2$ & $1,937.3$ & $1,979.9$ & $1,689.2$ & $1,948.8$ & $2,183.9$ & $2,431.2$ & $2,697.2$ & $2,977.4$ & $3,275.3$ \\
\hline Bank reserves & 644.5 & 882.4 & 929.2 & $1,072.7$ & $1,055.3$ & $1,093.8$ & $1,225.8$ & $1,364.6$ & $1,513.9$ & $1,671.1$ & $1,838.4$ \\
\hline Cash & 69.3 & 113.4 & 149.9 & 214.5 & 204.3 & 218.8 & 245.2 & 272.9 & 302.8 & 334.2 & 367.7 \\
\hline Deposits at the central bank & 575.2 & 769.0 & 779.3 & 858.1 & 851.0 & 875.1 & 980.6 & $1,091.6$ & $1,211.1$ & $1,336.9$ & $1,470.7$ \\
\hline Contribution to growth of reserve money & \multicolumn{11}{|c|}{ (Percent change in beginning-of-period reserve money) } \\
\hline Reserve money & 11.0 & 11.9 & 24.3 & 6.5 & -4.3 & 10.9 & 12.1 & 11.3 & 10.9 & 10.4 & 10.0 \\
\hline Net foreign assets & 49.0 & 18.2 & 30.1 & -11.1 & 7.7 & 9.1 & 3.9 & 9.2 & 4.7 & 7.2 & 6.9 \\
\hline Net domestic assets & -38.0 & -6.2 & -5.9 & 17.6 & -12.0 & 1.8 & 8.1 & 2.2 & 6.2 & 3.2 & 3.1 \\
\hline Domestic credit & -55.7 & -10.9 & -5.8 & -13.5 & -25.8 & 20.3 & -2.2 & -0.8 & -0.8 & 0.1 & 0.1 \\
\hline Claims on government (net) & -55.4 & -11.0 & -5.8 & -13.5 & -26.0 & 21.6 & -1.1 & 0.2 & 0.1 & 0.1 & 0.1 \\
\hline Claims on government (net) ${ }^{1}$ & -60.3 & -11.0 & 15.8 & -10.9 & -23.4 & 21.6 & -1.1 & 0.2 & 0.1 & 0.1 & 0.1 \\
\hline Advances to the government in foreign currencies ${ }^{2}$ & 5.0 & 0.0 & -21.7 & -2.6 & -2.6 & 0.0 & 0.0 & 0.0 & 0.0 & 0.0 & 0.0 \\
\hline Claims on banks (net) ${ }^{3}$ & 2.4 & 0.0 & 0.0 & 0.0 & 0.0 & 0.0 & 0.0 & 0.0 & 0.0 & 0.0 & 0.0 \\
\hline Claims on private and public enterprises & 0.2 & 0.1 & 0.0 & 0.0 & 0.2 & -1.3 & -1.1 & -1.0 & -0.9 & 0.0 & 0.0 \\
\hline Claims on public enterprises & 0.0 & 0.0 & 0.0 & 0.0 & 0.0 & -1.2 & -1.1 & -1.0 & -0.9 & 0.0 & 0.0 \\
\hline Claims on private enterprises & 0.2 & 0.1 & 0.0 & 0.0 & 0.2 & 0.0 & 0.0 & 0.0 & 0.0 & 0.0 & 0.0 \\
\hline Claims on foreign exchange bureaus ${ }^{4}$ & -2.9 & 0.0 & 0.0 & 0.0 & 0.0 & 0.0 & 0.0 & 0.0 & 0.0 & 0.0 & 0.0 \\
\hline Other items (net) & 17.7 & 4.7 & 0.0 & 31.1 & 13.8 & -18.5 & 10.4 & 2.9 & 7.0 & 3.0 & 3.1 \\
\hline \multicolumn{12}{|l|}{ Memorandum items: } \\
\hline \multicolumn{12}{|l|}{ Twelve-month change (percent) } \\
\hline Reserve money & 11.0 & 11.9 & 24.3 & 6.5 & -4.3 & 10.9 & 12.1 & 11.3 & 10.9 & 10.4 & 10.0 \\
\hline Net foreign asset & 107.4 & 21.3 & 32.6 & -11.3 & 7.8 & 8.2 & 3.6 & 9.1 & 4.8 & 7.8 & 7.6 \\
\hline Net domestic asset & -70.0 & -42.5 & -77.8 & $1,302.2$ & -886.3 & -16.1 & -96.7 & -886.6 & 361.7 & 44.2 & 33.4 \\
\hline \multicolumn{12}{|l|}{ Of which: } \\
\hline Claims on government (net) & -122.2 & 121.6 & 32.5 & 70.8 & 135.9 & -45.8 & 4.8 & -1.1 & -0.7 & -0.9 & -0.4 \\
\hline Claims on banks (net) & -427.7 & 0.0 & 0.0 & 0.0 & 0.0 & 0.0 & 0.0 & 0.0 & 0.0 & 0.0 & 0.0 \\
\hline Claims on public and private enterprises & 2.5 & 1.2 & 0.0 & 0.0 & 3.6 & -19.9 & -24.6 & -32.6 & -48.4 & 0.0 & 0.0 \\
\hline Claims on foreign exchange bureaus & -23.0 & 0.0 & 0.0 & 0.0 & 0.0 & 0.0 & 0.0 & 0.0 & 0.0 & 0.0 & 0.0 \\
\hline Other items (net) & -148.4 & 90.0 & -0.4 & 440.4 & 195.4 & -85.0 & 351.2 & 24.6 & 52.6 & 16.5 & 15.9 \\
\hline MDRI debt relief (millions of dalasis) $)^{5}$ & & & & -306.8 & -335.3 & & & & & & \\
\hline Gross international reserves (millions of U.S. dollars) & 84.0 & 96.6 & 118.6 & 120.2 & 141.6 & 153.0 & 160.0 & 173.0 & 177.0 & 186.0 & 194.0 \\
\hline
\end{tabular}

Sources: Gambian authorities.

${ }^{1}$ Excluding advances to the government in foreign currencies.

${ }^{2}$ These advances reflect previously unrecorded public spending and borrowing in 2001, financed by the CBG, and previously unrecorded depletion of foreign exchange reserves in 2001-03 as reported by the authorities on October 28, 2003.

${ }^{3}$ Advances to commercial banks and commercial banks' holdings of central bank bills.

${ }^{4}$ Claims on foreign exchange bureaus reflect the delayed delivery of foreign currency purchased on a spot basis.

${ }^{5}$ Based on the current exchange rate; however, the CBG and the government agreed to use the accounting exchange rate prevailing in April 2007 to arrive at D393.9 million as the MDRI deposits. 
Table 5A. The Gambia: Balance of Payments, 2004-13

\begin{tabular}{|c|c|c|c|c|c|c|c|c|c|c|c|}
\hline & $\begin{array}{r}2004 \\
\text { Est. }\end{array}$ & $\begin{array}{r}2005 \\
\text { Est. }\end{array}$ & $\begin{array}{r}2006 \\
\text { Est. }\end{array}$ & $\begin{array}{r}2007 \\
2 n d r e v .\end{array}$ & $\begin{array}{r}2007 \\
\text { Est. }\end{array}$ & $\begin{array}{r}2008 \\
\text { Proj. }\end{array}$ & $\begin{array}{r}2009 \\
\text { Proj. }\end{array}$ & $\begin{array}{r}2010 \\
\text { Proj. }\end{array}$ & $\begin{array}{r}2011 \\
\text { Proj. }\end{array}$ & $\begin{array}{r}2012 \\
\text { Proj. }\end{array}$ & $\begin{array}{r}2013 \\
\text { Proj. }\end{array}$ \\
\hline \multicolumn{12}{|l|}{ 1. Current account } \\
\hline A. Goods and Services & -84.1 & -104.9 & -87.6 & -118.6 & -105.3 & -148.1 & -162.8 & -176.6 & -186.4 & -196.2 & -208.1 \\
\hline Trade balance & -105.9 & -142.6 & -138.1 & -182.1 & -171.5 & -215.8 & -232.3 & -250.5 & -271.1 & -291.5 & -313.9 \\
\hline $\begin{array}{l}\text { Exports, f.o.b. } \\
\text { Groundnuts/groundnut products } \\
\text { Other domestic exports } \\
\text { Reexports } \\
\text { Imports, f.o.b. }\end{array}$ & $\begin{array}{r}96.8 \\
16.9 \\
9.0 \\
71.0 \\
-202.8\end{array}$ & $\begin{array}{r}80.9 \\
2.0 \\
5.2 \\
73.7 \\
-223.4\end{array}$ & $\begin{array}{r}84.0 \\
10.2 \\
7.2 \\
66.6 \\
-222.2\end{array}$ & $\begin{array}{r}87.8 \\
12.6 \\
7.8 \\
67.4 \\
-270.0\end{array}$ & $\begin{array}{r}91.4 \\
5.8 \\
6.7 \\
78.9 \\
-262.9\end{array}$ & $\begin{array}{r}101.5 \\
5.4 \\
7.3 \\
88.8 \\
-317.3\end{array}$ & $\begin{array}{r}106.1 \\
5.1 \\
7.9 \\
93.1 \\
-338.4\end{array}$ & $\begin{array}{r}112.6 \\
6.0 \\
8.6 \\
98.0 \\
-363.1\end{array}$ & $\begin{array}{r}119.0 \\
6.3 \\
9.3 \\
103.4 \\
-390.0\end{array}$ & $\begin{array}{r}125.1 \\
6.6 \\
10.1 \\
108.3 \\
-416.6\end{array}$ & $\begin{array}{r}130.7 \\
6.4 \\
11.0 \\
113.4 \\
-444.6\end{array}$ \\
\hline $\begin{array}{l}\text { For domestic use } \\
\text { Of which: oil products } \\
\text { For reexport }\end{array}$ & $\begin{array}{r}-131.8 \\
-23.2 \\
-71.0\end{array}$ & $\begin{array}{r}-149.7 \\
-29.8 \\
-73.7\end{array}$ & $\begin{array}{r}-155.5 \\
-16.1 \\
-66.6\end{array}$ & $\begin{array}{r}-202.6 \\
-26.6 \\
-67.4\end{array}$ & $\begin{array}{r}-184.1 \\
-28.5 \\
-78.9\end{array}$ & $\begin{array}{r}-228.4 \\
-42.1 \\
-88.8\end{array}$ & $\begin{array}{r}-245.3 \\
-40.7 \\
-93.1\end{array}$ & $\begin{array}{r}-265.0 \\
-42.6 \\
-98.0\end{array}$ & $\begin{array}{r}-286.7 \\
-44.9 \\
-103.4\end{array}$ & $\begin{array}{r}-308.3 \\
-47.4 \\
-108.3\end{array}$ & $\begin{array}{r}-331.3 \\
-50.0 \\
-113.4\end{array}$ \\
\hline Services balance & 21.8 & 37.6 & 50.6 & 63.5 & 66.3 & 67.7 & 69.6 & 73.8 & 84.6 & 95.3 & 105.8 \\
\hline $\begin{array}{l}\text { Travel income } \\
\text { Other services } \\
\text { Reexport earnings }\end{array}$ & $\begin{array}{r}53.1 \\
-66.2 \\
35.0\end{array}$ & $\begin{array}{r}67.5 \\
-66.2 \\
36.3\end{array}$ & $\begin{array}{r}75.4 \\
-67.7 \\
42.9\end{array}$ & $\begin{array}{r}81.2 \\
-59.4 \\
41.8\end{array}$ & $\begin{array}{r}84.0 \\
-57.2 \\
39.4\end{array}$ & $\begin{array}{r}89.9 \\
-66.6 \\
44.4\end{array}$ & $\begin{array}{r}97.5 \\
-74.4 \\
46.5\end{array}$ & $\begin{array}{r}105.8 \\
-81.0 \\
49.0\end{array}$ & $\begin{array}{r}114.8 \\
-81.8 \\
51.7\end{array}$ & $\begin{array}{r}124.5 \\
-83.4 \\
54.2\end{array}$ & $\begin{array}{r}135.1 \\
-86.0 \\
56.7\end{array}$ \\
\hline B. Income & -35.0 & -43.5 & -48.0 & -46.9 & -46.8 & -45.9 & -43.0 & -41.2 & -40.6 & -38.7 & -40.6 \\
\hline Income & -35.0 & -43.5 & -48.0 & -46.9 & -46.8 & -45.9 & -43.0 & -41.2 & -40.6 & -38.7 & -40.6 \\
\hline C. Current transfers & 94.6 & 78.8 & 77.3 & 95.5 & 71.8 & 82.2 & 95.4 & 106.6 & 109.9 & 114.5 & 120.3 \\
\hline $\begin{array}{l}\text { Remittances } \\
\text { Private transfers } \\
\text { Official transfers }\end{array}$ & $\begin{array}{r}52.4 \\
8.0 \\
34.2\end{array}$ & $\begin{array}{r}47.7 \\
7.4 \\
23.7\end{array}$ & $\begin{array}{r}51.6 \\
9.3 \\
16.3\end{array}$ & $\begin{array}{r}52.2 \\
9.3 \\
34.0\end{array}$ & $\begin{array}{r}52.5 \\
11.5 \\
7.8\end{array}$ & $\begin{array}{r}56.6 \\
9.8 \\
15.8\end{array}$ & $\begin{array}{l}59.3 \\
10.0 \\
26.1\end{array}$ & $\begin{array}{l}62.1 \\
10.3 \\
34.2\end{array}$ & $\begin{array}{l}65.0 \\
10.5 \\
34.4\end{array}$ & $\begin{array}{l}68.0 \\
10.8 \\
35.6\end{array}$ & $\begin{array}{l}71.2 \\
11.1 \\
38.0\end{array}$ \\
\hline $\begin{array}{l}\text { Current account (ex. official transfers) } \\
\text { Current account (inc. official transfers) }\end{array}$ & $\begin{array}{l}-58.8 \\
-24.6\end{array}$ & $\begin{array}{l}-93.3 \\
-69.6\end{array}$ & $\begin{array}{l}-74.6 \\
-58.3\end{array}$ & $\begin{array}{r}-104.1 \\
-70.1\end{array}$ & $\begin{array}{l}-88.1 \\
-80.3\end{array}$ & $\begin{array}{l}-127.6 \\
-111.8\end{array}$ & $\begin{array}{l}-136.5 \\
-110.3\end{array}$ & $\begin{array}{l}-145.5 \\
-111.3\end{array}$ & $\begin{array}{l}-151.4 \\
-117.0\end{array}$ & $\begin{array}{l}-156.0 \\
-120.4\end{array}$ & $\begin{array}{l}-166.4 \\
-128.4\end{array}$ \\
\hline $\begin{array}{l}\text { 2. Capital and financial account } \\
\text { A. Capital account } \\
\text { Capital transfers } \\
\text { Debt forgiveness (IDA/AfDF-MDRI relief) }\end{array}$ & & & & $\begin{array}{r}387.1 \\
14.4 \\
362.5\end{array}$ & $\begin{array}{r}377.4 \\
14.9 \\
362.5\end{array}$ & & & & & & \\
\hline B. Financial account & 48.0 & 103.1 & 77.5 & -306.4 & -263.6 & 110.6 & 105.3 & 115.9 & 116.7 & 125.2 & 134.1 \\
\hline $\begin{array}{l}\text { Private capital } \\
\text { Direct investment (net) } \\
\text { Other investment (net) }\end{array}$ & $\begin{array}{r}25.0 \\
56.6 \\
-31.6\end{array}$ & $\begin{array}{l}77.6 \\
61.2 \\
16.4\end{array}$ & $\begin{array}{r}50.5 \\
74.2 \\
-23.7\end{array}$ & $\begin{array}{r}40.2 \\
60.0 \\
-19.8\end{array}$ & $\begin{array}{l}92.5 \\
80.6 \\
11.9\end{array}$ & $\begin{array}{l}83.4 \\
70.2 \\
13.1\end{array}$ & $\begin{array}{l}67.2 \\
67.3 \\
-0.1\end{array}$ & $\begin{array}{r}72.7 \\
71.0 \\
1.6\end{array}$ & $\begin{array}{r}82.2 \\
74.9 \\
7.3\end{array}$ & $\begin{array}{l}95.5 \\
78.8 \\
16.7\end{array}$ & $\begin{array}{r}111.8 \\
83.0 \\
28.8\end{array}$ \\
\hline $\begin{array}{l}\text { Official loans (net) } \\
\text { Loans } \\
\text { Amortization }\end{array}$ & $\begin{array}{r}23.0 \\
37.3 \\
-14.4\end{array}$ & $\begin{array}{r}25.5 \\
39.8 \\
-14.4\end{array}$ & $\begin{array}{r}26.9 \\
43.9 \\
-17.0\end{array}$ & $\begin{array}{r}15.9 \\
36.5 \\
-20.6\end{array}$ & $\begin{array}{r}6.4 \\
23.6 \\
-17.2\end{array}$ & $\begin{array}{r}27.3 \\
35.9 \\
-8.6\end{array}$ & $\begin{array}{r}38.0 \\
46.8 \\
-8.8\end{array}$ & $\begin{array}{r}43.3 \\
51.8 \\
-8.5\end{array}$ & $\begin{array}{r}34.5 \\
45.3 \\
-10.8\end{array}$ & $\begin{array}{r}29.7 \\
41.8 \\
-12.1\end{array}$ & $\begin{array}{r}22.3 \\
37.3 \\
-15.0\end{array}$ \\
\hline Other capital (IDA, AfDF loans) & & & & -362.5 & -362.5 & & & & & & \\
\hline Capital and financial account balance & 48.0 & 103.1 & 77.5 & 80.7 & 113.8 & 110.6 & 105.3 & 115.9 & 116.7 & 125.2 & 134.1 \\
\hline Errors and omissions & 7.9 & -20.5 & 5.2 & 0.0 & -1.6 & 0.0 & 0.0 & 0.0 & 0.0 & 0.0 & 0.0 \\
\hline Overall balance & 31.3 & 13.0 & 24.4 & 10.6 & 31.9 & -1.1 & -5.1 & 4.6 & -0.4 & 4.8 & 5.7 \\
\hline $\begin{array}{l}\text { Financing } \\
\text { Change in gross official reserves (increase is }-\mathrm{v} \epsilon \\
\text { Use of IMF resources (net) } \\
\text { Repayments } \\
\text { Disbursements } \\
\text { Exceptional financing } 1 /\end{array}$ & $\begin{array}{r}-31.3 \\
-21.7 \\
-11.2 \\
-11.2 \\
0.0 \\
1.6\end{array}$ & $\begin{array}{r}-13.0 \\
-12.6 \\
-2.0 \\
-2.0 \\
0.0 \\
1.6\end{array}$ & $\begin{array}{r}-24.4 \\
-22.0 \\
-4.0 \\
-4.0 \\
0.0 \\
1.6\end{array}$ & $\begin{array}{r}-10.6 \\
-1.6 \\
-11.1 \\
-17.1 \\
6.0 \\
2.1\end{array}$ & $\begin{array}{r}-31.9 \\
-22.9 \\
-11.1 \\
-17.1 \\
6.0 \\
2.1\end{array}$ & $\begin{array}{r}1.1 \\
-11.5 \\
6.0 \\
0.0 \\
6.0 \\
6.6\end{array}$ & $\begin{array}{r}5.1 \\
-7.0 \\
6.1 \\
0.0 \\
6.1 \\
6.0\end{array}$ & $\begin{array}{r}-4.6 \\
-13.0 \\
3.0 \\
0.0 \\
3.0 \\
5.3\end{array}$ & $\begin{array}{r}0.4 \\
-4.0 \\
0.0 \\
0.0 \\
0.0 \\
4.4\end{array}$ & $\begin{array}{r}-4.8 \\
-9.0 \\
0.0 \\
0.0 \\
0.0 \\
4.2\end{array}$ & $\begin{array}{r}-5.7 \\
-8.0 \\
-1.9 \\
-1.9 \\
0.0 \\
4.2\end{array}$ \\
\hline \multicolumn{12}{|l|}{ Memorandum items: } \\
\hline $\begin{array}{l}\text { Gross official reserves (end of period) } \\
\text { Millions of U.S. dollars } \\
\text { Months of imports, c.i.f. }\end{array}$ & $\begin{array}{r}84.0 \\
4.3\end{array}$ & $\begin{array}{r}96.6 \\
4.5\end{array}$ & $\begin{array}{r}118.6 \\
5.5\end{array}$ & $\begin{array}{r}120.2 \\
4.8\end{array}$ & $\begin{array}{r}141.5 \\
5.5\end{array}$ & $\begin{array}{r}153 \\
4.9\end{array}$ & $\begin{array}{r}160 \\
4.8\end{array}$ & $\begin{array}{r}173 \\
4.9\end{array}$ & $\begin{array}{r}177 \\
4.7\end{array}$ & $\begin{array}{r}186 \\
4.6\end{array}$ & $\begin{array}{r}194 \\
4.5\end{array}$ \\
\hline Nominal GDP (millions U.S. dollars) & 401.0 & 461.3 & 507.7 & 648.4 & 643.5 & 779.3 & 843.0 & 913.2 & 990.7 & 1068.5 & 1151.9 \\
\hline
\end{tabular}

rities; IMF staff estimates

$1 /$ Includes debt relief from Paris Club; interim relief from multilaterals is treated as grants. 
Table 5B. The Gambia: Balance of Payments, 2004-13

\begin{tabular}{|c|c|c|c|c|c|c|c|c|c|c|c|}
\hline & $\begin{array}{c}2004 \\
\text { Est. }\end{array}$ & $\begin{array}{l}2005 \\
\text { Est. }\end{array}$ & $\begin{array}{c}2006 \\
\text { Est. }\end{array}$ & $\begin{array}{c}2007 \\
2 n d \text { rev. }\end{array}$ & $\begin{array}{c}2007 \\
\text { Est. }\end{array}$ & $\begin{array}{l}2008 \\
\text { Proj. }\end{array}$ & $\begin{array}{l}2009 \\
\text { Proj. }\end{array}$ & $\begin{array}{l}2010 \\
\text { Proj. }\end{array}$ & $\begin{array}{l}2011 \\
\text { Proj. }\end{array}$ & $\begin{array}{l}2012 \\
\text { Proj. }\end{array}$ & $\begin{array}{l}2013 \\
\text { Proj. }\end{array}$ \\
\hline \multicolumn{12}{|l|}{ 1. Current account } \\
\hline A. Goods and Services & -21.0 & -22.7 & -17.2 & -18.3 & -16.4 & -19.0 & -19.3 & -19.3 & -18.8 & -18.4 & -18.1 \\
\hline Trade balance & -26.4 & -30.9 & -27.2 & -28.1 & -26.7 & -27.7 & -27.6 & -27.4 & -27.4 & -27.3 & -27.3 \\
\hline Exports, f.o.b. & 24.1 & 17.5 & 16.6 & 13.5 & 14.2 & 13.0 & 12.6 & 12.3 & 12.0 & 11.7 & 11.3 \\
\hline Groundnuts/groundnut products & 4.2 & 0.4 & 2.0 & 1.9 & 0.9 & 0.7 & 0.6 & 0.7 & 0.6 & 0.6 & 0.6 \\
\hline Other domestic exports & 2.2 & 1.1 & 1.4 & 1.2 & 1.0 & 0.9 & 0.9 & 0.9 & 0.9 & 0.9 & 1.0 \\
\hline Reexports & 17.7 & 16.0 & 13.1 & 10.4 & 12.3 & 11.4 & 11.0 & 10.7 & 10.4 & 10.1 & 9.8 \\
\hline Imports, f.o.b. & -50.6 & -48.4 & -43.8 & -41.6 & -40.9 & -40.7 & -40.1 & -39.8 & -39.4 & -39.0 & -38.6 \\
\hline For domestic use & -32.9 & -32.5 & -30.6 & -31.2 & -28.6 & -29.3 & -29.1 & -29.0 & -28.9 & -28.8 & -28.8 \\
\hline Of which: oil products & -5.8 & -6.5 & -3.2 & -4.1 & -4.4 & -5.4 & -4.8 & -4.7 & -4.5 & -4.4 & -4.3 \\
\hline For reexport & -17.7 & -16.0 & -13.1 & -10.4 & -12.3 & -11.4 & -11.0 & -10.7 & -10.4 & -10.1 & -9.8 \\
\hline Services balance & 5.4 & 8.2 & 10.0 & 9.8 & 10.3 & 8.7 & 8.3 & 8.1 & 8.5 & 8.9 & 9.2 \\
\hline Travel income & 13.2 & 14.6 & 14.8 & 12.5 & 13.0 & 11.5 & 11.6 & 11.6 & 11.6 & 11.7 & 11.7 \\
\hline Other services & -16.5 & -14.4 & -13.3 & -9.2 & -8.9 & -8.5 & -8.8 & -8.9 & -8.3 & -7.8 & -7.5 \\
\hline Reexport earnings & 8.7 & 7.9 & 8.5 & 6.4 & 6.1 & 5.7 & 5.5 & 5.4 & 5.2 & 5.1 & 4.9 \\
\hline B. Income & -8.7 & -9.4 & -9.5 & -7.2 & -7.3 & -5.9 & -5.1 & -4.5 & -4.1 & -3.6 & -3.5 \\
\hline Income & -8.7 & -9.4 & -9.5 & -7.2 & -7.3 & -5.9 & -5.1 & -4.5 & -4.1 & -3.6 & -3.5 \\
\hline C. Current transfers & 23.6 & 17.1 & 15.2 & 14.7 & 11.2 & 10.5 & 11.3 & 11.7 & 11.1 & 10.7 & 10.4 \\
\hline Remittances & 13.1 & 10.3 & 10.2 & 8.1 & 8.2 & 7.3 & 7.0 & 6.8 & 6.6 & 6.4 & 6.2 \\
\hline Private transfers & 2.0 & 1.6 & 1.8 & 1.4 & 1.8 & 1.3 & 1.2 & 1.1 & 1.1 & 1.0 & 1.0 \\
\hline Official transfers & 8.5 & 5.1 & 3.2 & 5.2 & 1.2 & 2.0 & 3.1 & 3.7 & 3.5 & 3.3 & 3.3 \\
\hline Current account (ex. official transfers) & -14.7 & -20.2 & -14.7 & -16.1 & -13.7 & -16.4 & -16.2 & -15.9 & -15.3 & -14.6 & -14.4 \\
\hline Current account (inc. official transfers) & -6.1 & -15.1 & -11.5 & -10.8 & -12.5 & -14.3 & -13.1 & -12.2 & -11.8 & -11.3 & -11.1 \\
\hline \multicolumn{12}{|l|}{ 2. Capital and financial account } \\
\hline A. Capital account & & & & 59.7 & 58.6 & & & & & & \\
\hline Capital transfers & & & & 2.2 & 2.3 & & & & & & \\
\hline Debt forgiveness (IDA/AfDF-MDRI relief) & & & & 55.9 & 56.3 & & & & & & \\
\hline B. Financial account & 12.0 & 22.3 & 15.3 & -47.3 & -41.0 & 14.2 & 12.5 & 12.7 & 11.8 & 11.7 & 11.6 \\
\hline Private capital & 6.2 & 16.8 & 10.0 & 6.2 & 14.4 & 10.7 & 8.0 & 8.0 & 8.3 & 8.9 & 9.7 \\
\hline Direct investment (net) & 14.1 & 13.3 & 14.6 & 9.3 & 12.5 & 9.0 & 8.0 & 7.8 & 7.6 & 7.4 & 7.2 \\
\hline Other investment (net) & -7.9 & 3.6 & -4.7 & -3.1 & 1.8 & 1.7 & 0.0 & 0.2 & 0.7 & 1.6 & 2.5 \\
\hline Official loans (net) & 5.7 & 5.5 & 5.3 & 2.5 & 1.0 & 3.5 & 4.5 & 4.7 & 3.5 & 2.8 & 1.9 \\
\hline $\begin{array}{l}\text { Loans } \\
\text { o/w guaranteed }\end{array}$ & 9.3 & 8.6 & 8.6 & 5.6 & 3.7 & 4.6 & 5.6 & 5.7 & 4.6 & 3.9 & 3.2 \\
\hline Amortization & -3.6 & -3.1 & -3.3 & -3.2 & -2.7 & -1.1 & -1.0 & -0.9 & -1.1 & -1.1 & -1.3 \\
\hline Other capital (IDA, AfDF loans) & & & & -55.9 & -56.3 & & & & & & \\
\hline Capital and financial account balance & 12.0 & 22.3 & 15.3 & 12.4 & 17.7 & 14.2 & 12.5 & 12.7 & 11.8 & 11.7 & 11.6 \\
\hline Errors and omissions & 2.0 & -4.4 & 1.0 & 0.0 & -0.3 & 0.0 & 0.0 & 0.0 & 0.0 & 0.0 & 0.0 \\
\hline Overall balance & 7.8 & 2.8 & 4.8 & 1.6 & 5.0 & -0.1 & -0.6 & 0.5 & 0.0 & 0.5 & 0.5 \\
\hline Financing & -7.8 & -2.8 & -4.8 & -1.6 & -5.0 & 0.1 & 0.6 & -0.5 & 0.0 & -0.5 & -0.5 \\
\hline Change in gross official reserves (increase is -ve) & -5.4 & -2.7 & -4.3 & -0.2 & -3.6 & -1.5 & -0.8 & -1.4 & -0.4 & -0.8 & -0.7 \\
\hline Use of IMF resources (net) & -2.8 & -0.4 & -0.8 & -1.7 & -1.7 & 0.8 & 0.4 & 0.0 & 0.0 & -0.2 & -0.3 \\
\hline Repayments & -2.8 & -0.4 & -0.8 & -2.6 & -2.7 & 0.0 & 0.0 & 0.0 & 0.0 & -0.2 & -0.3 \\
\hline Disbursements & 0.0 & 0.0 & 0.0 & 0.9 & 0.9 & 0.8 & 0.4 & 0.0 & 0.0 & 0.0 & 0.0 \\
\hline Exceptional financing ${ }^{1}$ & 0.4 & 0.3 & 0.3 & 0.3 & 0.3 & 0.8 & 0.6 & 0.5 & 0.4 & 0.4 & 0.4 \\
\hline \multicolumn{12}{|l|}{$\begin{array}{l}\text { Memorandum items: } \\
\text { Gross official reserves (end of period) }\end{array}$} \\
\hline Percent of GDP & 20.9 & 20.9 & 23.4 & 18.5 & 22.0 & 19.6 & 19.0 & 18.9 & 17.9 & 17.4 & 16.8 \\
\hline Months of imports, c.i.f. & 4.3 & 4.5 & 5.5 & 4.8 & 5.5 & 4.9 & 4.8 & 4.9 & 4.7 & 4.6 & 4.5 \\
\hline Nominal GDP (millions U.S. dollars) & 401 & 461 & 508 & 648 & 644 & 779 & 843 & 913 & 991 & 1068 & 1152 \\
\hline
\end{tabular}

Sources: Gambian authorities; IMF staff estimates and projections.

${ }^{1}$ Includes debt relief from Paris Club; interim relief from multilaterals is treated as grants. 
Table 6. The Gambia: Proposed Schedule of Disbursements

\begin{tabular}{llll}
\hline & \multicolumn{2}{c}{ Disbursement } & \\
\cline { 2 - 3 } \multicolumn{1}{c}{ Date } & SDRs & $\begin{array}{c}\text { Percent } \\
\text { of quota }^{1}\end{array}$ & Conditions \\
\hline September 8, 2008 & $2,000,000$ & 6.43 & Completion of third review (end-March 2008 test date) \\
January 31, 2009 & $2,000,000$ & 6.43 & Completion of fourth review (end-September 2008 test date) \\
July 31, 2009 & $2,000,000$ & 6.43 & Completion of fifth review (end-March 2009 test date) \\
January 30, 2010 & $2,000,000$ & 6.43 & Completion of sixth review (end-September 2009 test date) \\
Total & $8,000,000$ & 25.72 & \\
\hline
\end{tabular}

${ }^{1}$ The Gambia's quota is SDR 31.10 million. 
Table 7. The Gambia: Millennium Development Goals, 1990-2006 1/

\begin{tabular}{|c|c|c|c|c|}
\hline & 1990 & 1995 & 2000 & 2006 \\
\hline \multicolumn{5}{|l|}{ Goal 1: Eradicate extreme poverty and hunger } \\
\hline Employment to population ratio, 15+, total (\%) & 68 & 68 & 67 & 66 \\
\hline Employment to population ratio, $15-24$, total (\%) & 52 & 52 & 51 & 51 \\
\hline Income share held by the lowest $20 \%$ & 4.4 & .. & 4.0 & . \\
\hline Malnutrition prevalence, weight for age (\% of children under 5) & .. & .. & 15.4 & . \\
\hline Poverty headcount ratio at national poverty line ( $\%$ of population) & 64 & .. & 57.6 & . \\
\hline Prevalence of undernurishment ( $\%$ of population) & 22 & 31 & 30 & 29 \\
\hline \multicolumn{5}{|l|}{ Goal 2: Achieve universal primary education } \\
\hline Primary completion rate, total (\% of relevant age group) & 43 & 44 & 53 & 63 \\
\hline School enrollment, primary (\% net) & 46 & .. & 64 & 62 \\
\hline \multicolumn{5}{|l|}{ Goal 3: Promote gender equality and empower women } \\
\hline Proportion of seats held by women in national parliament (\%) & 8 & .. & 2 & 13 \\
\hline Ratio of girls to boys in primary education (\%) & 70 & 79 & 87 & 108 \\
\hline Ratio of girls to boys in secondary education (\%) & 50 & 60 & 69 & 90 \\
\hline Ratio of girls to boys in tertiary education (\%) & .. & .. & 30 & 24 \\
\hline Share of women employed in the nonagricultural sector (\%) & 20.9 & .. & .. & . \\
\hline \multicolumn{5}{|l|}{ Goal 4: Reduce child mortality } \\
\hline Immunization, measles ( $\%$ of children ages $12-23$ months) & 86 & 91 & 85 & 95 \\
\hline Infant mortality rate (per 1,000 live births) & 103 & 102 & 94 & 84 \\
\hline Under 5 mortality rate (per 1,000$)$ & 153 & 149 & 132 & 113 \\
\hline \multicolumn{5}{|l|}{ Goal 5: Improve maternal health } \\
\hline Adolescent fertility rate (births per 1,000 women ages $15-19$ ) & .. & 131 & 120 & 106 \\
\hline Births attended by skilled health staff ( $\%$ of total) & 44 &.. & 55 & 57 \\
\hline Contraceptive prevalence ( $\%$ of women ages $15-49$ ) & 12 & .. & 10 & . \\
\hline Maternal mortality ratio (per 100,000 live births) & .. & .. & .. & 690 \\
\hline Pregnant women receiving maternal care $(\%)$ & .. & .. & 91 & 98 \\
\hline \multicolumn{5}{|l|}{ Goal 6: Combat HIV/AIDS, malaria, and other diseases } \\
\hline Children with fever receiving antimalarial drugs ( $\%$ of children under 5 with fever) & .. & .. & 55 & 63 \\
\hline Incidence of tuberculosis (per 100,000 people) & 183 & 204 & 226 & 257 \\
\hline Prevalence of HIV, female (\% of population aged $15-49$ ) &.. &.. & .. & 1.7 \\
\hline Prevalence of HIV, total (\% of population aged $15-49)$ & .. & .. & .. & 2.4 \\
\hline Tuberculosis cases detected under DOTS (\%) & .. & 74 & 67 & 64 \\
\hline \multicolumn{5}{|l|}{ Goal 7: Ensure environmental sustainability } \\
\hline Annual freshwater withdrawals, total (\% of internal resources) & .. & & & . \\
\hline $\mathrm{CO} 2$ emissions (kg per PPP \$ GDP)) & 0.3 & 0.2 & 0.2 & 0.2 \\
\hline $\mathrm{CO} 2$ emissions (metric tons per capita) & 0.2 & 0.2 & 0.2 & 0.2 \\
\hline Forest area ( $\%$ of total land area) & 44 &.. & 46 & 47 \\
\hline Access to improved sanitation ( $\%$ of population) & .. & 53 & 53 & 53 \\
\hline Access to an improved water source ( $\%$ of population) & .. & 82 & 82 & 82 \\
\hline Marine protected areas ( $\%$ of surface area) & .. & .. & .. & 2 \\
\hline Nationally protected areas ( $\%$ of total land area) & .. & .. & .. & 3.5 \\
\hline \multicolumn{5}{|l|}{ Goal 8: Develop a global partnership for development } \\
\hline Aid per capita (current US\$) & 101 & 39 & 35 & 45 \\
\hline Debt service (\% of exports) & 21.8 & 15.0 & 11.0 & 11.6 \\
\hline Internet users (per 1,000 people) & .. & 0.0 & 0.9 & 3.6 \\
\hline Mobile phone subscribers (per 1,000 people) & .. & 0.1 & 0.4 & 24.3 \\
\hline Telephone mainlines (per 1,000 people) & 0.6 & 1.7 & 2.4 & 3.2 \\
\hline \multicolumn{5}{|l|}{ Other } \\
\hline Fertility rate, total (births per woman) & 6.0 & 5.7 & 5.3 & 4.8 \\
\hline GNI per capita, Atlas method (current US\$) & 300 & 330 & 310 & 290 \\
\hline GNI, Atlas method (current US\$) (billions) & 0.3 & 0.4 & 0.4 & 0.5 \\
\hline Gross capital formation (\% of GDP) & 22.3 & 20.2 & 17.4 & 24.3 \\
\hline Life expectancy at birth, total (years) & 51 & 55 & 57 & 59 \\
\hline Population, total (millions) & 1 & 1.2 & 1.4 & 1.7 \\
\hline Trade (\% of GDP) & 131.5 & 122 & 104.8 & 110.2 \\
\hline
\end{tabular}

Source: World Development Indicators database.

1/ Figures in italics refer to periods other than those specified. 


\section{Appendix I}

Banjul, The Gambia

July 30, 2008

Mr. Dominique Strauss-Kahn

Managing Director

International Monetary Fund

Washington, D.C. 20431

Dear Mr. Strauss-Kahn:

1. The Gambia's three-year Poverty Reduction and Growth Facility (PRGF) arrangement was approved by the IMF's Executive Board in February 2007. The first and second reviews were completed on August 29 and December 19, 2007, respectively. The attached Memorandum of Economic and Financial Policies (MEFP) reviews progress in implementing the Government's PRGF-supported program in 2007 and during the first half of 2008, and sets out the policies that the government will pursue in the rest of 2008. We are committed to continue maintaining macroeconomic stability and fostering reforms conducive to higher growth and poverty reduction.

2. Performance under the program has been quite strong. All the quantitative performance criteria for end-March 2008 were met, and four out of eight structural performance criteria scheduled for implementation between December 2007 and July 2008 were fully implemented on time. Of the remainder, two were met with a slight delay while the other two were only partially implemented. On the basis of corrective measures that have been taken (see MEFP, paragraphs 12-14), we request waivers for the nonobservance of four performance criteria.

3. In support of our policies described in the MEFP, the Government of The Gambia requests the completion of the third review and the release of the fourth disbursement under the PRGF arrangement in an amount equivalent to SDR 2 million.

4. The government believes that the policies set forth in the attached MEFP are adequate to achieve the objectives of its program. However, it will stand ready to take any additional measures that may become appropriate to meet these objectives. The Gambia will consult with the IMF on the adoption of these measures and in advance of revisions to policies contained in the MEFP, in accordance with the Fund's policies on such consultation. The fourth and fifth reviews under the PRGF arrangement are expected to be completed by no later than end-January 2009, and end-July 2009, respectively. 
5. The government intends to make the contents of this letter and the attached MEFP and Technical Memorandum of Understanding available to the public. Therefore, it authorizes the IMF to arrange for these documents to be posted on the IMF website following Executive Board conclusion of the review.

Sincerely yours,

$/ \mathrm{s} /$

Mousa Gibril Bala-Gaye

Secretary of State

Department of State for Finance and Economic Affairs /s/

Momodou Bamba Saho

Governor

Central Bank of The Gambia

Attachments: Memorandum of Economic and Financial Policies

Technical Memorandum of Understanding 


\section{Attachment I \\ Memorandum of Economic and Financial Policies}

\section{INTRODUCTION}

1. This memorandum updates the Government of The Gambia's economic and financial program under the three-year Poverty Reduction and Growth Facility (PRGF) arrangement with the International Monetary Fund (IMF). The program, which was approved by the Executive Board of the IMF in February 2007, aims at consolidating macroeconomic stability and fostering the conditions for sustaining high economic growth and reducing poverty. The first and second reviews were successfully completed in August and December 2007, respectively. The Gambia reached completion point under the enhanced Initiative for Heavily Indebted Poor Countries (HIPC) in December 2007 and is receiving debt relief under both the HIPC Initiative and the Multilateral Debt Relief Initiative (MDRI).

\section{RECENT ECONOMIC DEVELOPMENTS}

2. Growth has remained strong but is expected to slow down in 2008. Real GDP grew at about $6 \frac{1}{2}$ percent a year in 2006 and 2007, led by telecommunications, tourism and construction. Tourist arrivals are on pace to increase by about 10 percent in the 2007/08 season compared to 2006/07; the average length of stay remained roughly the same, albeit with a changing pattern and type of accommodation. Growth in the agriculture sector declined in 2007 due to poor rains during the early part of the crop season. For 2008, overall growth is projected to slow to 5.5 percent, mainly on account of the impact of adverse international developments and dalasi appreciation on tourism and remittances.

3. After rising sharply in 2007, inflation has slowed in 2008. Driven by rising world food prices, the annual rate of inflation on a year-on-year basis rose from less than 1 percent in December 2006 to 6-7 percent during most 2007. Appreciation of the dalasi and relatively tight monetary policy helped to contain the price shocks. Year-on-year inflation was 2.2 percent in June 2008. The 12-month moving average rate fell from 5.2 percent in December 2007 to 4 percent in June 2008. In order to help keep the price of rice affordable, the government eliminated the sales tax on rice imports in May 2008; the tax had been reduced from 15 percent to 5 percent in July 2007.

4. The overall fiscal balance improved in 2007. Gains from tax administration reforms boosted revenues, while shortfalls in the projected level of external financing led to a marked contraction in capital expenditures. In particular, a dispute between the contractor and the supervising consultant delayed grant disbursements for a large European Union (EU)supported roads project. The problem has now been resolved and implementation of the project is expected to proceed. The stock of domestic government debt fell from 32 percent of GDP at end-2006 to 28 percent of GDP at end-2007, compared to nearly 30 percent projected at the time of the second PRGF review. 
5. Performance weakened somewhat in the first quarter of 2008 compared with the first quarter of 2007. Domestic revenues were down 3.5 percent while domestic expenditures increased by 16.5 percent. Nonetheless, the government was able to achieve a surplus of D51 million on the basic balance. On the revenue side, taxes on international trade fell 11 percent. At the same time, wages increased 35 percent in line with the program to help retain and attract skilled manpower to the government. This increase was partially offset by a 21 percent reduction in external interest payments due to HIPC and MDRI relief. In 2007, the government cleared more domestic arrears than programmed, and is on target to clear the remaining stock before the end of 2008 .

6. The government increased retail prices of petroleum products in May 2008. The prices per liter were increased from D28 to D32 for diesel, from D30 to D33 for petrol, and from D21 to D26 for kerosene. Petrol and diesel prices were last increased in January 2006, and kerosene in June 2007. The government is committed to adjusting the prices as needed to avoid burdening the budget by subsidizing these products. However, kerosene will continue to be cross-subsidized by the other products.

7. Growth in broad money slowed down significantly in 2007 and through May 2008. Growth fell from 26 percent in 2006 to 7 percent in 2007 as a result of improved government finances. Credit to the private sector was stagnant during most of 2007. Reserve money contracted by 4 percent in 2007 after expanding by 24 percent in 2006, and fell further by 6 percent in the first half of 2008. In March 2008, in response to indications of tight liquidity conditions, and against a backdrop of falling inflation, the CBG reduced the unremunerated statutory minimum reserve requirement of banks from 16 percent to 14 percent.

8. Since the sharp appreciation during the third quarter of 2007, fluctuation of the dalasi in response to changing supply and demand conditions in the foreign exchange market has become more pronounced. The dalasi appreciated by 32 percent by end-September 2007 against the U.S. dollar, mainly due to a change in market sentiments when many banks decided to unwind their long net open positions at the same time during August and September 2007. However, a reversal of the trend occurred during the fourth quarter 2007 when the dalasi depreciated by 18 percent. The CBG acted to restore stability by actively intervening in the market during August-November. Against a backdrop of strong inflow of remittances, less need for foreign exchange to service debt (due to debt relief), and tight liquidity conditions, the dalasi appreciated by 8 percent during the first half of 2008 .

9. The external current account deficit (including official transfers) widened from $11 \frac{1}{2}$ percent of GDP in 2006 to $12 \frac{1}{2}$ percent in 2007. The current account deficits were financed largely by inflows of foreign direct investment (FDI). In 2007, FDI included US $\$ 28.5$ million in privatization proceeds, reflecting part payment (out of a total of US\$35 million) for the sale of 50 percent of GAMTEL. 
10. The financial sector is relatively sound and competition has been increasing. With the entry of two new banks since October 2007, there are now ten commercial banks operating in The Gambia and two more have been granted approval-in-principle. Generally, the banks are profitable and adequately capitalized. However, their gross earnings in 2007 were significantly lower than in 2006, reflecting, in large part, revaluation losses associated with the marked appreciation of the dalasi. The risk-weighted capital adequacy ratio has stayed in the 22-23 percent range since the first quarter of 2007, against the minimum requirement of 8 percent. Nonperforming loans fell during the first quarter of 2008 to 10 percent of gross loans (compared to about 13 percent in the fourth quarter of 2007) as banks have been making serious efforts in loan recovery and in restructuring loans. In order to satisfy the demand for an Islamic instrument, the CBG introduced the short-dated Sharia-compliant Sukuk Al-Salaam in November 2007.

\section{Performance Under the Program}

11. All the quantitative performance criteria for end-March 2008 were met (Table A1). New external loans contracted during October 2007-March 2008 amounted to US\$10.9 million in net present value terms, compared to an indicative target of US\$20.7 million.

12. Four out of eight structural performance criteria were met (Table A2). Of the rest, two (submission of special audit reports on program monetary data by December 2007 and June 2008) were implemented with a slight delay, while the others were only partially met. As was the case in earlier reviews, the delays on the special audit reports reflected delays experienced by the external auditor in obtaining confirmations from some of the CBG's correspondent banks abroad.

13. With respect to the establishment of a central register of capital expenditure commitments, information was collected in different formats and varying degrees of completeness for individual projects. A uniform format has now been adopted and information gaps are being filled. Completion of the register has been established as a prior action for completion of the third review (Table A2).

14. A credit reference bureau has been established but is not yet fully operational. Banks are providing information to the Bureau but concerns about the legal basis for sharing information have slowed down implementation of the project. The Financial Institutions Act (2003) prohibits the sharing of information on individual customers of banks. The Act is under review, inter alia, to address this shortcoming. In view of uncertainty about the legislative calendar, this performance criterion has been reset to end-March 2009 to provide a realistic timeframe for implementation.

15. On the basis of the remedial actions that the government and the CBG have taken, the government requests waivers for the non-observance of the four performance criteria indicated above. 
16. With respect to structural benchmarks, there has been a delay in the submission of the Auditor-General's report on the government's 2000-04 accounts to the national assembly (scheduled for end-December 2007). In January 2008, the Auditor-General provided an interim report to the Department of State for Finance and Economic Affairs (DoSFEA) to allow the Treasury Directorate an opportunity to respond to some queries and correct portions of the accounts. DoSFEA responded in May, and the Auditor-General now expects to complete a review and submit his final report to the national assembly by end-October 2008.

17. Work is progressing well on rebasing the national accounts to 2004 prices and on beginning to estimate GDP by the expenditure approach. Full implementation of this benchmark is now expected by end-September 2008 .

\section{MediUM-TERM ObJECTIVES AND STRATEGY}

18. The government's medium-term objectives and strategy are contained in The Gambia's second Poverty Reduction Strategy Paper (PRSP II). PRSP II, which covers 200711, provides the framework for poverty reduction efforts in The Gambia and also sets the development agenda towards meeting the Millennium Development Goals (MDGs) by 2015. Strategic priorities include: (i) macroeconomic stability and effective public resource management; (ii) promotion of pro-poor growth and employment through private sector development; and (iii) improved provision of basic social services. Successful implementation of PRSP II requires significant strengthening of capacity in the civil service, improvements in the budget process (formulation, monitoring), and the mobilization of highly concessional external resources.

19. A Diagnostic Trade Integration Study (DTIS) led by the World Bank was approved by the government in August 2007, following the validation of its findings by stakeholders. It found that re-export trade (on which a large part of the economy and public finances depend) was in decline as a result of The Gambia losing some of the advantages which made it an attractive regional entrepot. In particular, harmonization of imports and sales taxes in the region and improvements in ports and customs operations in Senegal and other neighboring countries have eroded The Gambia's competitive edge. The DTIS contains a wide range of recommendations to enhance The Gambia's international competitiveness through sectorspecific reforms and cross-cutting measures to improve the investment climate. Key areas of focus include infrastructure (e.g., transport, energy), tourism, and agricultural export diversification. The government is seeking funding to implement the highest priority recommendations of the DTIS, including those related to reinforcing trade facilitation services.

20. The Gambia has preserved macroeconomic stability in the last two and a half years with strong growth, relatively low inflation, and adequate international reserves. The government will continue to maintain prudent macroeconomic policies in the face of 
challenging global economic and financial conditions. The main objectives and assumptions underlying the medium term macroeconomic framework are:

- real GDP growth rates of 5.5-6.5 percent a year;

- inflation below 6 percent;

- basic fiscal balance surpluses of 1-2 percent of GDP;

- a reduction in government's domestic debt from 28 percent at end-2007 to 16 percent at end-2010;

- external current account deficits (including official transfers) around 10 percent of GDP; and

- international reserves equivalent to 4-5 months of imports.

21. The baseline scenario takes into account the importance of preserving fiscal discipline, containing inflation, and ensuring the sustainability of the external current account. It seeks to reduce the burden of domestic debt to ease upward pressure on interest rates and create fiscal space for poverty-reducing expenditures. The current account path reflects the medium-term projections of capital inflows.

22. Resources freed by debt relief under the HIPC and MDR Initiatives will be used to increase poverty-reducing expenditures, in line with PRSP II priorities. They will be allocated among various uses, including increased social sector expenditures, reduced domestic debt, and civil service pay and pension reform.

23. The government has established a Task Force to prepare recommendations for a comprehensive civil service reform program for The Gambia, drawing on studies supported by the World Bank and other donors. The main aim of the reform is to rebuild the capacity of the civil service to formulate and implement policies needed for effective delivery of public services. A heavy loss of professional and managerial staff has undermined the effectiveness of the civil service. To reverse this, the reform program is expected to address issues related to pay and benefits, as well as career development. In the short run (within one year), the reform will focus on pay reform, developing a pension reform strategy, and strengthening payroll and establishment controls. Over the medium-term (2-5 years), activities will include functional review of all departments, overhaul of job descriptions and schemes of service, and implementation of the pension reform.

24. The CBG will continue to take steps to promote the development and deepening of the financial sector. The CBG will encourage competition among financial institutions in order to lower interest rate spreads, increase the provision of services, expand the branch network in all parts of the country, and enhance growth and development. The CBG has taken a number of legal initiatives to promote a safe and vibrant financial system in The Gambia. To that end, it: (1) is preparing a draft Banking Act to replace the Financial 
Institutions Act 2003; (2) has prepared a new Non-Bank Financial Institutions Bill to encourage nonbanks to provide financial services; (3) has proposed amendments to Mortgage and Sheriff Acts that will reduce legal risks for banks, thereby promoting lending to the private sector. The CBG Board also has approved two new initiatives to promote foreign exchange operations. They are: (1) a code of conduct of foreign exchange market operators; and (2) establishing a system of market makers in foreign exchange, similar to the primary market dealership in treasury bills. The CBG will continue to implement the Prompt Corrective Action (PCA) framework, introduced in March 2007, to guide and help troubled banks. The CBG is setting up a new Financial Intelligence Unit (FIU) to combat money laundering.

\section{POLICIES FOR THE REST OF 2008}

25. A major challenge for economic management in the second half of 2008 will be coping with the impact of rising world food and oil prices. Thus far, appreciation of the dalasi has helped contain the impact of the price increases. At the same time, it has adversely affected the prospects for the upcoming tourist season.

\section{A. Fiscal policy}

26. Revenue is broadly on track to meet the targets set in the government's 2008 budget. On the expenditure side, efforts will be made to speed up the disbursements for externally financed capital expenditures. The government may consider expanding social programs (e.g., school feeding program) to mitigate the impact of rising world food prices on the poor.

27. Preparation for the 2009 budget has begun with the issuance of the call circular in June. Key elements of the preparations will include government decisions on civil service and pension reforms. The government is also in discussions with the AfDB, EU, and the World Bank for assistance in the form of budget support.

28. The CBG and DoSFEA have enhanced their coordination of fiscal and monetary policies. Further to signing a memorandum of understanding last year to guide domestic debt management and monetary operations, the government has undertaken the following to strengthen the CBG's financial position and its ability to conduct monetary policy. It will: (1) take over a nonperforming loan of the CBG to the Gambia National Petroleum Corporation of D136.9 million and clear the loan within four years at equal installments with the first one beginning in the last quarter of 2008; (2) continue to replenish the CBG's capital by D20 million each year to the total of D100 million; of which, contributions for 2006-07 and half of the amount for 2008 have already been made; (3) reach agreement with the CBG in the near future on the timing and the amount of replenishment of the treasury bill special deposit account. Currently, this account has a very low balance, thereby limiting the CBG's options to conduct monetary policy. DoSFEA and the CBG will agree on concrete proposals by end-October 2008 . 


\section{B. Monetary and Exchange Rate Policies}

29. The CBG will continue to use a money targeting framework to pursue its price stability objective. It will also use its rediscount rate to signal changes in its policy stance. In setting the rediscount rate, the CBG will analyze developments in the economy and the inflation outlook. It will strengthen the short-term liquidity forecasting framework through efforts to improve forecasts of government revenues and expenditures.

30. The exchange rate of the dalasi is market determined. The CBG will intervene in the foreign exchange market from time to time to: (1) accumulate foreign reserve assets to meet targets or prevent reserves from being depleted, which is essential to build investor confidence and strengthen the government's debt repayment capacity and external liquidity conditions; (2) correct misalignment of the exchange rate of the domestic currency; and (3) calm disorderly markets, including exchange rate volatility and market illiquidity.

31. The second half of 2008 will be a challenging period for monetary policy making as The Gambia faces the impact of the increase in international food and oil prices. CBG policy will continue to be based on maintaining price stability. The CBG will use all the available instruments at its disposal to reach this goal.

\section{External Debt}

32. The HIPC and MDRI relief has significantly reduced The Gambia's external debt burden. In net present value terms, the stock of debt fell from $\$ 439$ million (68 percent of GDP) to $\$ 165$ million (24 percent of GDP) at end-2007. Paris Club creditors have confirmed that they will provide the debt relief they promised. The government is making efforts to obtain debt relief from all non-Paris Club bilateral creditors.

33. The government is committed to the careful management of external debt to maintain external stability. To this end, it promotes a strategy of donor engagement and prudent debt management to attain PRSP priorities without compromising debt sustainability. It will be prudent with new borrowing and will respect the indicative targets for 2008 ( $\$ 50$ million) under the program. The limit for 2009 (\$25 million) will be revisited in the context of program reviews, which will take into account the result of the government's own debt sustainability analysis (DSA). Support is being sought from the Commonwealth Secretariat for technical assistance to undertake a DSA and to build capacity. Results of the DSA are expected by October 2008. The government will also ensure that new loans have a minimum grant element of 45 percent.

34. The results of the DSA will feed into the design of a national debt management strategy which will be prepared by end-February 2009. The strategy will be in line with best international practice and will incorporate the following principles: (1) a clear definition of debt management objectives; (2) a well-defined institutional framework; (3) an adequate 
mechanism to prevent the accumulation of arrears and monitor contingent liabilities; and (4) a commitment to implement cost effective cash management policies.

\section{PRSP II Implementation and Aid Effectiveness}

35. PRSP II was the focus of a Round Table Conference on The Gambia held in London in February 2008. The government has established mechanisms to monitor and analyze progress implementing PRSP II. In particular, the government has prepared a Results Matrix providing quantitative indicators and targets that can be matched to development results. A draft of the Annual Poverty Report for 2007 has been completed, and a final version will be published after it has been validated by stakeholders. A draft of the Poverty Reduction Expenditure Report for 2007 is also under preparation, and is expected to be finalized by end-August 2008.

36. Under the government's ownership and leadership, an in-country Development Partners Coordination and Consultation Mechanism has been established under the Chairmanship of the Secretary of State for Finance and Economic Affairs. This mechanism is to facilitate and deepen development dialogue with The Gambia's partners, bring about positive changes to aid practices, and encourage more effective use of aid resources for greater development impact for the implementation of PRSP II.

37. At the first consultation and coordination meeting held in April 2008, the government presented to its development partners a draft Aid Effectiveness Action Plan. The Action Plan adapted the Paris Declaration principles of Ownership, Alignment, Harmonization, Management for Results, and Mutual Accountability to the situation of The Gambia. The meeting noted the positive steps taken by some multilateral donors to have a joint Country Assistance Strategy for The Gambia and called on other development partners to develop similar joint and harmonized programming activities for the country. Consultation and coordination meetings will be held quarterly. At the next meeting, the Aid Effectiveness Action Plan will be finalized. The government informed development partners of plans to hold sector specific consultation and coordination meetings starting with Health and Education in the course of 2008.

\section{Program Monitoring AND ACCess Level}

38. The program will continue to be monitored based on agreed quantitative targets (Table A1), a set of structural performance criteria and benchmarks (Table A3), and program reviews. The quantitative financial targets for end-September 2008 and end-March 2009 are performance criteria; and those for end-December 2008, and end-June 2009 are indicative targets. The fourth and fifth program reviews are scheduled to be completed by end-January 2009 and end-July 2009, respectively. Definition of all targeted variables and reporting requirements are contained in the attached technical memorandum of understanding (TMU). 
39. The CBG has strengthened its internal control and audit. In lieu of the special audits, the $\mathrm{CBG}$ proposes to implement alternative measures that would provide assurances to the IMF that financial controls at the CBG have been strengthened. These measures include: (1) preparation of pro-forma financial statements for 2007 based on the International Financial Reporting Standards (IFRS), which should be reviewed by the CBG's external auditor, and (2) internal audit reports verifying the accuracy of monetary program data for test dates (September 2008 and March 2009). As the contract of the current external auditors expired after the audit of the 2007 financial statements, the CBG and the Auditor-General (who is responsible for appointing the CBG's external auditor) are discussing an audit rotation and selection policy appropriate for The Gambia's circumstances (few options available locally).

40. To ensure effective monitoring of program implementation, the PRGF Monitoring Committee, headed by the Secretary of State for Finance and Economic Affairs will continue to meet regularly to review performance under the program. It will also ensure that data are reported to the IMF as per the schedule agreed in the TMU and will provide any other information deemed necessary or requested by IMF staff in order to monitor the program. The committee will also take remedial actions in the event there are gaps or delays in reporting reliable statistics.

41. The government will monitor the impact of rising world food and oil prices on The Gambia's balance of payments in the coming months. If the balance of payments deteriorates significantly on account of adverse price developments, the government may request an augmentation of the level of access to IMF resources under the PRGF arrangement. The government intends to take this up with the next review mission, currently scheduled for October/November 2008.

\section{ReQuests For TeChNiCAl ASSISTANCE AND FSAP}

42. The government is grateful for the technical assistance that the IMF has provided to The Gambia. It welcomes the resumption of assistance in public financial management with the appointment of a new regional advisor based in Liberia whose work will complement the work of other macro-fiscal and debt management experts in DoSFEA, who are being supported under an AfDB technical assistance grant.

43. The government and the CBG welcome the continuation of assistance on financial, balance of payments, and national accounts statistics. The government requests that the IMF provide assistance to the Gambia Bureau of Statistics in the compilation of producer prices.

44. Technical assistance from the IMF has also contributed to the strengthening of banking supervision at the CBG. In view of the increasing number of banks and the introduction of new products in the financial sector, we request The Gambia be considered for an FSAP. 
Table A1. The Gambia: Quantitative Targets and Projections, End-December 2006 to End-June 2009

\begin{tabular}{|c|c|c|c|c|c|c|c|c|c|c|c|}
\hline & \multirow{4}{*}{$\begin{array}{c}\frac{2006}{2} \\
\text { End-Dec. } \\
\text { Act. }\end{array}$} & \multirow{2}{*}{\multicolumn{2}{|c|}{$\begin{array}{c}2007 \\
\text { End-Dec. }{ }^{1} \\
\end{array}$}} & \multirow{2}{*}{\multicolumn{2}{|c|}{ End-Mar. }} & \multicolumn{3}{|l|}{2008} & \multirow{4}{*}{ 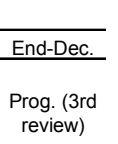 } & \multicolumn{2}{|c|}{2009} \\
\hline & & & & & & End-J & & & & End-Mar. & End-Jun. \\
\hline & & $\begin{array}{l}\text { Prog. } \\
\text { (2nd }\end{array}$ & & $\begin{array}{l}\text { Prog. } \\
\text { (2nd }\end{array}$ & & $\begin{array}{l}\text { Prog. } \\
\text { (2nd }\end{array}$ & & $\begin{array}{l}\text { Prog. } \\
\text { (3rd }\end{array}$ & & & \\
\hline & & review) & Act. & review) & Act. & review) & Act. & review) & & review) & review) \\
\hline & (Stock) & & & & & hange from & End-Dec & mber 2006) & & & \\
\hline Performance criteria ${ }^{2}$ & & & & & & & & & & & \\
\hline & & & & & & Millions of $\mathrm{c}$ & lalasis) & & & & \\
\hline Net domestic assets of the central bank (ceiling) & 38.7 & 504.5 & & 603.2 & & 581.7 & & -48.8 & -97.0 & 87.5 & 49.2 \\
\hline Adjusted for privatization proceeds ${ }^{3}$ & & 262.5 & -270.4 & 361.2 & -334.5 & 339.7 & -393.2 & & & & \\
\hline Basic balance (floor) ${ }^{4}$ & & 659.4 & 613.6 & 628.5 & 661.8 & 746.2 & & 793.4 & 859.3 & 932.5 & $1,005.8$ \\
\hline New external payments arrears of the central government (ceiling) ${ }^{5}$ & 0.0 & 0.0 & 0.0 & 0.0 & 0.0 & 0.0 & 0.0 & 0.0 & 0.0 & 0.0 & 0.0 \\
\hline & & & & & & lions of U. & dollars) & & & & \\
\hline Net usable international reserves (floor) & 94.9 & 12.3 & & 15.8 & & 16.2 & & 27.0 & 36.5 & 34.8 & 36.2 \\
\hline Adjusted for privatization proceeds ${ }^{6}$ & & 23.3 & 32.0 & 26.8 & 27.5 & 27.2 & 29.0 & & & & \\
\hline New nonconcessional debt contracted or guaranteed by the & & & & & & & & & & & \\
\hline government with original maturity of more than one year (ceiling) ${ }^{7}$ & 0.0 & 0.0 & 0.0 & 0.0 & 0.0 & 0.0 & 0.0 & 0.0 & 0.0 & 0.0 & 0.0 \\
\hline Outstanding stock of external public debt with original maturity of one & & & & & & & & & & & \\
\hline year or less (ceiling) ${ }^{8}$ & 0.0 & 0.0 & 0.0 & 0.0 & 0.0 & 0.0 & 0.0 & 0.0 & 0.0 & 0.0 & 0.0 \\
\hline Indicative targets & & & & & & & & & & & \\
\hline & & & & & & Millions of $\mathrm{c}$ & alasis) & & & & \\
\hline Domestic budgetary arrears & 561.5 & -440.2 & -455.5 & -486.5 & -547.7 & -532.5 & $\ldots$ & -561.5 & -561.5 & -561.5 & -561.5 \\
\hline & & & & & & lions of U.S & dollars) & & & & \\
\hline Net present value of new contracted external debt (cumulative ceiling) & & 4.2 & & 20.7 & 10.9 & 20.7 & $\ldots$ & 31.7 & 31.7 & 38.6 & 38.6 \\
\hline Memorandum item: & & & & & & & & & & & \\
\hline Program exchange rate $(\mathrm{D} / \$)$ & & 22.0 & 22.0 & 22.0 & 22.0 & 22.0 & 22.0 & 22.0 & 22.0 & 22.0 & 22.0 \\
\hline Privatization proceeds (\$ millions) & $\ldots$ & 17.5 & 28.5 & 17.5 & 28.5 & 17.5 & 28.5 & 35.0 & 35.0 & 35.0 & 35.0 \\
\hline Privatization proceeds ( $\mathrm{D}$ millions at program exchange rate) & $\ldots$ & 385.0 & 627.0 & 385.0 & 627.0 & 385.0 & 627.0 & 770.0 & 770.0 & 770.0 & 770.0 \\
\hline
\end{tabular}

Source: IMF staff estimates.

${ }^{1} \mathrm{MDRI}$ debt relief took place in the fourth quarter of 2007.

${ }^{2}$ March 2008, September 2008, and March 2009 are performance criteria; December 2007, June 2008, December 2008, and June 2009 are indicative targets.

${ }^{3}$ Adjusted upward (downward) by the dalasi equivalent of the extent to which actual receipts fall short of (exceed) projected level of privatization receipts.

${ }^{4}$ Defined as domestic revenue minus expenditure and net lending, excluding externally financed capital expenditure.

${ }^{5}$ To be applied on a continuous basis.

${ }^{6}$ Adjusted upward (downward) by the extent to which actual receipts exceed (fall short of) projected level of privatization receipts.

${ }^{7}$ External debt contracted or guaranteed other than that with a grant element equivalent to 45 percent or more, calculated using a discount rate based on the

Organization for Economic Corporation and Development (OECD) commercial interest reference rates (CIRRs). Excludes borrowing from the IMF.

${ }^{8}$ Excluding normal import-related credits.

${ }^{9}$ Cumulative from October 1, 2007. 
Table A2. The Gambia: Structural Conditionality for December 2007-July $2008^{1}$

\begin{tabular}{|c|c|c|}
\hline Measure & Target Date & Status \\
\hline \multicolumn{3}{|l|}{ Public financial management and accountability } \\
\hline \multicolumn{3}{|l|}{ 1. Auditing of government accounts } \\
\hline (i) Submit to the national assembly the audited accounts for 2000-04 (B) & End-December 2007 & Not met \\
\hline (ii) Submit to the Auditor-General the accounts for 2006 (PC) & End-December 2007 & Met \\
\hline $\begin{array}{l}\text { 2. Issue a comprehensive monthly budget execution report, including } \\
\text { information on commitments, with a one-month lag (PC) }\end{array}$ & Monthly & Met \\
\hline $\begin{array}{l}\text { 3. Establish a central register of capital expenditure commitments related to externally } \\
\text { financed projects for ongoing and new projects (PC) }\end{array}$ & End-April 2008 & Partially met \\
\hline $\begin{array}{l}\text { 4. Establish a central register of capital expenditure commitments related to } \\
\text { externally financed ongoing and new projects (PA) }\end{array}$ & July 15,2008 & Met \\
\hline 5. Prepare a national debt strategy after receiving TA $(B)$ & End-July 2008 & Not met \\
\hline \multicolumn{3}{|l|}{ Central bank governance and operational independence } \\
\hline $\begin{array}{l}\text { 6. Implement the action plan to bring government borrowing from the CBG in line } \\
\text { With the limit under the CBG Act (PC) }\end{array}$ & End-December 2007 & Met \\
\hline \multicolumn{3}{|l|}{$\begin{array}{l}\text { 7. Provide to Fund staff special audit report on monetary program data at program } \\
\text { test dates: }\end{array}$} \\
\hline 8. Complete a gap analysis and establish an Action Plan for moving to IFRS (PC) & December 2007 & Met \\
\hline \multicolumn{3}{|l|}{ Financial deepening } \\
\hline 9. Make the credit reference bureau operational (PC) & End-March 2008 & Partially met \\
\hline \multicolumn{3}{|l|}{ Statistics } \\
\hline 10. Publish quarterly balance of payments statistics, with one quarter lag Q1 (B) & $\begin{array}{l}\text { (i) End-December (2007Q3 data) } \\
\text { (ii) End-March } 2008 \text { (2007Q4 data) } \\
\text { (iii) End-June } 2008 \text { (2008Q1 data) }\end{array}$ & $\begin{array}{l}\text { Met } \\
\text { Met with delay } \\
\text { Met with delay }\end{array}$ \\
\hline $\begin{array}{l}\text { 11. Rebase the national accounts to } 2004 \text { prices and begin estimating GDP by } \\
\text { expenditure components (B) }\end{array}$ & June 2008 & Partially met \\
\hline
\end{tabular}

${ }^{1} \mathrm{PA}, \mathrm{PC}$, and $\mathrm{B}$ denote prior action, performance criterion, and benchmark, respectively. 
Table A3. The Gambia: Structural Conditionality for September 2008-September $2009^{1}$ Measure

Target Date

Macro Rationale

\section{Public financial management and accountability}

1. Auditing of government accounts

(i) Submit to the Auditor-General the accounts for 2007 (PC)

(i) Submit to the national assembly the audited accounts for 2005, 2006, and 2007 (B)

2. Prepare a national debt strategy after receiving TA (B)

End-September 2008

End-September 2009

End-February 2009
To improve fiscal accountability and hence control.

To ensure debt sustainability.

\section{Strengthening financial controls at the Central Bank of The Gambia}

3. Prepare pro-forma financial statements for 2007 based on IFRS and have End-September 2008 this reviewed by the CBG's external auditor (PC)

To enhance the central bank's internal controls and audit capacity, thereby reducing financial risk.

\section{Financial deepening}

4. Make the credit reference bureau operational (PC)

End-March 2009

To deepen the financial sector by removing an impediment to evaluation of risk.

\section{Statistics}

5. Publish quarterly balance of payments statistics, with one quarter lag

(i) End-September 2008 (2008Q2 data)

(ii) End-December 2008 (2008Q3 data)

(iii) End-March 2009

(2008Q4 data)

(iv) End-June 2009 (2009Q1 data)

September 2008
6. Rebase the national accounts to 2004 prices and begin estimating GDP by expenditure components $(B)$
To improve policy formulation through timely provision of economic statistics.

To improve policy formulation by providing additional information on the national accounts.

$\mathrm{PC}$ and $\mathrm{B}$ denote performance criterion and benchmark, respectively. 


\section{Attachment II \\ Technical Memorandum of Understanding}

(July 2008-September 2008)

\section{INTRODUCTION}

1. This memorandum sets out the understandings between the Gambian authorities and staff of the International Monetary Fund (IMF) regarding the definitions of quantitative performance criteria, indicative targets, structural performance criteria, and structural benchmarks that will be used to monitor the Poverty Reduction and Growth Facility (PRGF)supported program covering the period of 2007-09. It also sets out the related reporting requirements and describes the adjusters that will be applied to certain quantitative performance criteria of the program.

\section{Quantitative Performance Criteria}

\section{A. Net Domestic Assets of the Central Bank}

2. Definition: The net domestic assets of the CBG are defined as the difference between reserve money and the net foreign assets of the $\mathrm{CBG}$. Reserve money is defined as the sum of currency issued by the CBG (i.e., currency in circulation) and the deposits of commercial banks at the CBG. Net foreign assets are defined as foreign assets minus foreign liabilities. Foreign assets and foreign liabilities are defined as claims on nonresidents and liabilities to nonresidents, respectively.

3. For program monitoring purposes, in the calculation of the net domestic assets of the CBG, foreign assets and liabilities will be converted first into U.S. dollars at the prevailing cross-rates and then converted into dalasi using the D/USD program exchange rate of 22 . This is an accounting exchange rate only and should not be construed as a projection.

4. Adjuster: The net domestic assets of the CBG will be adjusted upward (downward) by the dalasi equivalent of the extent to which actual receipts fall short of (exceed) the projected level of privatization receipts.

5. Supporting material: Net domestic assets of the central bank will be transmitted as part of the balance sheet of the CBG (compiled based on the TMU rate) on a monthly basis within four weeks of the end of each month. For analytical purposes, the balance sheet of the CBG compiled on a current-rate basis will also be submitted.

6. Supporting material: The CBG will report data on privatization receipts in the currency it is received in as well as equivalent amounts in U.S. dollars and in dalasis on a monthly basis within two weeks of the end of the month. The Department of State for 
Finance and Economic Affairs (DoSFEA) will report data on a monthly basis within two week of the end of the month on expenditures made from the privatization receipts.

\section{B. Basic Balance of the Central Government}

7. Definition: The basic balance of the central government is defined as revenue (tax and nontax) minus total expenditure and net lending, excluding externally financed capital expenditure. Central government excludes local and regional governments and public enterprises.

8. Supporting material: Reporting on the basic balance will form part of the consolidated budget report described in paragraph 30 below.

\section{New External Payments Arrears of the Central Government}

9. Definition: External payments arrears are defined as the stock of external arrears on loans contracted or guaranteed by the central government, except on debts subject to rescheduling or a stock of debt operation. Debts subject to rescheduling include debts covered under traditional mechanisms (bilateral creditors, such as the Paris Club members) or HIPC. External payments arrears occur when undisputed interest and amortization payments on the above-referenced loans are not made within the terms of the debt contract or in conformity with the terms for interim relief provided under the enhanced HIPC Initiative. This performance criterion will be assessed on a continuous basis.

10. Supporting material: An accounting of nonreschedulable external arrears (if any) by creditor countries, with detailed explanations, will be transmitted on a monthly basis within four weeks of the end of each month. This accounting would include, separately, arrears owed by the central government and other public sector entities to Paris Club and non-ParisClub creditors.

\section{Net Usable International Reserves of the Central Bank of The Gambia}

11. Definition: Net usable international reserves (NIR) of the $\mathrm{CBG}$ are defined as the difference between usable reserve assets and reserve liabilities. Usable reserve assets are readily available claims on nonresidents denominated in foreign convertible currencies. They include the CBG holdings of SDRs, foreign currency cash, foreign currency securities, deposits abroad, and the country's reserve position at the IMF. Excluded are any assets that are pledged, collateralized, or otherwise encumbered, claims on residents, claims in foreign exchange arising from derivatives in foreign currencies vis-à-vis domestic currency (such as futures, forwards, swaps, and options), precious metals, assets in nonconvertible currencies, and illiquid assets (including capital shares in international organizations). Reserve liabilities are all foreign exchange liabilities to residents and nonresidents, including commitments to sell foreign exchange arising from derivatives (such as futures, forwards, swaps, and options), and all credit outstanding from the IMF. 
12. Adjuster: Net usable international reserves of the CBG will be adjusted upward (downward) by the extent to which actual receipts in foreign currency exceed (fall short of) the projected level of privatization receipts in foreign currency.

13. Supporting material: End-month data on net usable international reserves of the CBG will be transmitted within seven days of the end of each month. The CBG will identify the U.S. dollar equivalent of privatization receipts within net usable international reserves as a memorandum item.

\section{E. New Nonconcessional Debt Contracted or Guaranteed by the Central Government with Original Maturity of More Than one Year}

14. Definition: This target refers to new nonconcessional external debt with original maturity of more than one year contracted or guaranteed by the central government. It applies not only to debt as defined in point No. 9 of the Guidelines on Performance Criteria with Respect to Foreign Debt, adopted by the Executive Board of the IMF on August 24, 2000 (Decision No. 12274-00/85), but also to commitments contracted or guaranteed for which value has not been received. Excluded from this target are loans or purchases from the IMF and debts with a grant element of at least 45 percent.

15. ${ }^{1}$ Also excluded are two loans from the OPEC Fund for International Development with grant elements of 39.5 percent each, which were approved in the first half of 2007.

16. Supporting material: A comprehensive record, including a loan-by-loan accounting of all new concessional and nonconcessional debt contracted or guaranteed by the central government with detailed explanations, will be transmitted on a quarterly basis within four weeks of the end of each quarter. Nonconcessional external debt over one year includes financial leases and other instruments giving rise to external liabilities, contingent or otherwise, on nonconcessional terms.

\section{F. Outstanding Stock of External Public Debt with Original Maturity of One Year or Less}

17. Definition: This target refers to the stock of outstanding external public sector debt with original maturity of one year or less, owed or guaranteed by the central government. ${ }^{2}$

\footnotetext{
${ }^{1} \mathrm{~A}$ loan is concessional if its grant element is at least 35 percent, calculated on the basis of the commercial interest reference rates (CIRR) and following the methodology set out in staff paper on Limits on External Debt or Borrowing in Fund Arrangements - Proposed Change in Implementation of the Revised Guidelines approved by the IMF Executive Board on April 15, 1996.

2 The term "debt" has the meaning set forth in point No. 9 of the Guidelines on Performance Criteria with Respect to Foreign Debt, adopted by the Executive Board of the IMF on August 24, 2000 (Decision No. 12274-00/85).
} 
Public sector consists of the central and regional governments and other public agencies, including the CBG. Excluded from this target are normal import-related credits.

18. Supporting material: A comprehensive record of all external debt with original maturity of less than one year owed or contracted by the public sector, with detailed explanations, will be transmitted on a quarterly basis within four weeks of the end of each quarter.

\section{QUANTITATIVE INDICATIVE TARGETS}

\section{A. Domestic Budgetary Arrears}

19. Definition: Domestic budgetary arrears are defined as the sum of all bills that have been received by a central government spending unit or line ministry under the recurrent expenditure budget (including rents and utilities) or the development expenditure budget, and for which payment has not been made within 30 days. Arrears can be cleared in cash or through debt swaps.

20. Supporting material: A comprehensive record of all domestic budgetary arrears, with detailed explanations, will be transmitted on a quarterly basis within four weeks of the end of each quarter.

\section{B. Net Present Value of New Contracted External Debt}

21. Definition: The net present value (NPV) of new external debt contracted or guaranteed by the government from October 2007 onward is calculated by discounting the future stream of payments of debt service due by the country-specific commercial interest reference rates (CIRRs) as published by the Organization for Economic Cooperation and Development (OECD). The new external debt will be measured by the U.S. dollar nominal sum of all loan agreements that have been contracted. Disbursed debt will be converted to U.S. dollars, based on prevailing WEO test date exchange rates; for loans contracted but not yet disbursed, the profile disbursement will be measured at the actual exchange rate at the test date, based on the projected drawdown consistent with the medium-term fiscal framework as discussed with the Fund staff.

22. Supporting material:. Data on the NPV of the stock of outstanding external debt contracted or guaranteed by the government since October 2007 will be provided on a monthly basis within five weeks of the end of each month.

\section{Structural Performance Criteria And Benchmarks}

\section{A. Central Register of Capital Expenditure Commitments}

23. Definition: The register in the Central Project Management and Aid Coordination Directorate of the Department of State for Finance and Economic Affairs is deemed 
established when the directorate compiles and maintains an up-to-date database on capital expenditure commitments made by the government in relation to ongoing and new externally financed projects.

24. Supporting material: A comprehensive record of capital expenditure commitments made by the government in relation to ongoing and new externally financed projects will be transmitted on a quarterly basis within four weeks of the end of each quarter. The record shall explicitly identify commitments made for each project.

\section{B. Pro-Forma Financial Statements}

25. The CBG shall prepare pro-forma financial statements based on the International Financial Reporting Standards (IFRS) for the 2007 financial statement by end-September 2008. The pro-forma financial statement shall include IFRS-required disclosures and balances valued in accordance with the IFRS. It shall be reviewed by the external auditors, who shall prepare a report summarizing their findings from the review and recommended next steps to achieve full compliance.

\section{Credit Reference Bureau}

26. The Bureau is deemed operational when it is staffed, begins compiling a database on commercial bank customers, and commercial banks are able to share information from the database. The legal basis for sharing such information should be formalized by amending Section 60 of the Financial Institutions Act (2003).

\section{Quarterly Balance of Payments Statistics}

27. Supporting material: Quarterly balance of payments data transmitted to the IMF with a one quarter lag.

\section{E. National accounts}

28. Supporting material: Gambia Bureau of Statistics publication of national accounts series showing expenditure components (consumption, investment, net exports) as well as sector of origin of GDP, in both current and constant (2004) market prices.

\section{Other DATA REQUIREMENTS AND REPORTING STANDARDS}

29. In addition to providing the data needed to monitor program implementation in relation to the program's performance criteria, indicative targets, and benchmarks, as set out above, the authorities will transmit the following data within the time frame specified below: 


\section{A. Prices}

30. The monthly disaggregated consumer price index, including weights for each major category, with August $2004=100$, will be transmitted within four weeks of the end of each month.

\section{B. Government Accounts Data}

31. A monthly consolidated central government budget report (i.e., the analytical table) on budget execution during the month and cumulatively from the beginning of the year, will be transmitted to the IMF within four weeks of the end of the month. The report will comprise: (i) revenue data by major item, including tax (direct tax, taxes on domestic goods and services, and taxes on international trade) and nontax; (ii) external grants by type (e.g., project, program); (iii) details of recurrent expenditure (including data on wages and salaries, interest payments, and other charges); (iv) details of capital expenditure and net lending (including data on externally financed capital expenditure, expenditures from the Gambia Local Fund, and net lending); (v) the overall balance and the basic balance (defined in paragraph 7); and (vi) details of budget financing (including net domestic borrowing and its gross components, external grants, net external borrowing and its gross components, utilization of privatization proceeds, and arrears).

32. Net domestic borrowing by the central government over a given period is defined as the difference between the net domestic debt at the end of the period and the net domestic debt at the beginning of the period. The central government's net domestic debt is defined as: claims on the central government by the banking system minus deposits of the central government with the banking system plus claims by the nonbanking sector, including public enterprises. Central government excludes local and regional governments and public enterprises. The banking system comprises the CBG and commercial banks.

\section{Poverty Reducing Expenditures}

33. A monthly report on poverty-reducing expenditures, by functional and economic classifications, will be transmitted within four weeks of the end of each month. Povertyreducing expenditures comprise line items in the budget that have been specifically tagged as PRSP-related. For 2007, they include expenditure on the construction of trunk roads.

\section{Monetary Sector Data}

34. The balance sheets of the CBG, prepared on the basis of current and program exchange rates, will be transmitted on a monthly basis to the IMF within four weeks of the end of each month. The balance sheet should explicitly identify all claims on, and liabilities to, the government. Claims include overdrafts, holdings of treasury bills, interest and noninterest-bearing government bonds, advances to the government in foreign currency, and other claims. Liabilities include balances in the treasury main, treasury expenditure, 
consolidated revenue fund and other revenue accounts, treasury bill special deposit, privatization, special projects, foreign projects, and other deposit accounts.

35. The consolidated balance sheet of the commercial banks and a monetary survey (i.e., consolidation of the accounts of the CBG and the commercial banks), including foreign currency deposits held by residents of The Gambia with commercial banks, will be transmitted within four weeks of the end of each month.

36. The CBG will also forward, within four weeks of the end of each month, data on banks' reserves held at the $\mathrm{CBG}$ to meet statutory reserve requirements during the last week of each month (broken down by total reserves, and excess reserves or deficits). Data will be provided for each commercial bank as well as for the industry as a whole.

37. The CBG will also forward within four weeks of the end of each month, data on government borrowing from the CBG as defined in the CBG Act 2005. The data shall indicate the limit on government borrowing from the CBG based on the government's tax revenues in the preceding year.

\section{E. Treasury Bills}

38. Weekly data on the amount offered, issued, net issuance, over/under subscription, and yields (interest rates) of the various instruments will be transmitted on a monthly basis within seven days of the end of the month. Data on treasury bills outstanding (including information on the distribution by bank and nonbank holders) will be transmitted on a monthly basis within six weeks of the end of each month. The monthly Liquidity Management Report, reflecting the data as of the last working day of the month, will be transmitted within seven days after the end of each month.

\section{F. External Sector Data}

39. The following standards will be adhered to in reporting data on exchange rates: (i) the interbank market exchange rates, defined as the simple average of the weekly weighted average buying and selling rates, will be transmitted on a weekly basis within five business days of the end of the week; and (ii) the CBG's published monthly average and end-month exchange rates, including those for all currencies in which foreign assets and liabilities are denominated, will be transmitted within two weeks of the end of the month.

40. The CBG will also forward monthly data on volume of transactions (purchases, sales, and total) in the foreign exchange market by each major group of participants (CBG, commercial banks, and foreign exchange bureaus) in dalasis within two weeks of the end of the month. 


\section{G. CBG Report on Monetary Program Data}

41. The CBG shall forward a report prepared by the Internal Audit Department verifying the accuracy of monetary data submitted to the IMF. The report shall be submitted within one quarter after each test date. The first test date for which the report to be prepared is September 2008. 


\title{
INTERNATIONAL MONETARY FUND
}

\section{THE GAMBIA}

\section{Update on Joint IMF/IDA Debt Sustainability Analysis ${ }^{1}$}

\author{
Prepared by the Staff of the International Monetary Fund
}

Approved by Hugh Bredenkamp and Mark Plant

August 20, 2008

The updated Debt Sustainability Analysis (DSA) confirms that The Gambia remains at high risk of debt distress after receiving HIPC and MDRI debt relief. In particular, the NPV of external debt-to-exports ratio remains above its threshold of 100 percent and standard stress tests show that The Gambia remains vulnerable to shocks to GDP and the exchange rate. The fiscal DSA shows public debt declining due to recent fiscal consolidation.

\section{BACKGROUND}

1. This debt sustainability analysis (DSA) updates the last DSA presented to the Fund Board in December 2007 at The Gambia's completion point. This update was prepared by IMF staff and reviewed by staff of the World Bank. The DSA is based on debt and debt service data reconciled for the completion point under the HIPC/MDRI initiative. These data were updated to reflect newly available debt data and a revised macroeconomic framework resulting from the 2008 Article IV consultation.

\section{The Gambia reached the completion point under the enhanced HIPC Initiative} and qualified for debt relief under the MDRI on December 19, 2007. At the end of 2006, prior to completion point, the stock of nominal external public debt was US\$676.7 million (133.6 percent of GDP). Multilateral creditors accounted for 84 percent of this debt, with IDA as the largest creditor (39 percent of total outstanding debt). At end-2007, postcompletion point, the stock of external public debt fell to US\$299.4 million (46.5 percent of GDP). In January 2008, Paris Club creditors agreed to cancel outstanding claims (US\$13 million in NPV terms at end-2006) on The Gambia. Bilateral agreements have been signed with Paris Club creditors France and Norway and agreement has been reached with non-Paris Club creditor Kuwait. Agreements on the delivery of debt relief are still pending with Paris Club creditors Austria, the Netherlands, and the EU-IDA and with non-Paris Club creditors the Saudi Fund for Development, Taiwan Province of China, Libya, China, and

\footnotetext{
${ }^{1}$ The last DSA was presented to the Fund Executive Board on December 19, 2007 (IMF Country Report No. 08/109, Appendix I) and to the World Bank Executive Board on December 20, 2007 (Enhanced HIPC Completion Point Document and MDRI, Report No. 41413-GM).
} 
India. HIPC debt relief has been or is in the process of being delivered by all multilateral creditors with the exception of ECOWAS, which to date has not participated in the HIPC Initiative. $^{2}$

\section{Box 1: Baseline Macroeconomic Assumptions Underlying the DSA}

The medium-term assumptions in the baseline scenario are consistent with the IMF PRGF supported program. Key macroeconomic assumptions include continued robust growth, prudent fiscal and monetary policies, investment in infrastructure and tourism, and a scaling up of donor assistance.

Real GDP growth averaged $6 \frac{1}{2}$ percent over the past five years and is projected to remain at $5 \frac{1}{2}$ to 6 percent out to 2013. The main drivers of growth are expected to be tourism, construction, telecommunications, and banking. Inflation is projected to decline gradually from 6 percent in 2008 to 4 percent over the medium-term. This assumes the authorities maintain tight monetary policy to adjust to high food and energy costs over the medium to long-term.

Growth of exports of goods and services is expected to be driven by tourism and agricultural exports. Re-exports are expected to decline as a share of GDP as tariff harmonization and improvements in neighboring countries erode The Gambia's competitive advantage. Export growth is expected to average 6 $1 / 4$ percent from 2008-2013 while import growth is expected to average $9 \frac{1}{4}$ percent over the same period. Official transfers are expected to gradually recover to 3-4 percent of GDP over the medium-term. FDI is expected to remain strong while official loans fall from a peak of $4 \frac{1}{2}$ percent of GDP in 2009 to about $1-2$ percent of GDP over the medium- to long-term. The non-interest current account deficit is projected to gradually declines from a peak of 14 percent of GDP in 2008 to 11 percent of GDP in 2012 and $6 \frac{1}{4}$ percent of GDP in 2027.

The primary fiscal balance is projected to decline from a surplus of $5 \frac{1}{2}$ percent of GDP in 2007 to close to zero in 2027. The surplus is expected to drop in the near term due to a recovery in capital expenditures. Over the long-term, tax revenues are projected to remain close to 20 percent of GDP while poverty reducing expenditures are boosted in line with the fall in debt service payments. Donor support, including program and project assistance is expected to remain robust over the medium-term. Grant financing is expected to remain in the region of 3-4 percent of GDP over the medium-term before falling to an average of 2 percent over 2013-27. The grant element of new external borrowing is projected to remain at 45 percent.

\footnotetext{
${ }^{2}$ The debt relief agreement with the Islamic Development Bank has been negotiated and is in the process of being signed.
} 


\section{EXTERnal DebT Sustainability}

\section{A. Baseline}

3. Under the baseline scenario, all but one of the NPV of debt indicators will remain below their corresponding thresholds for $2008-27$ as a result of the HIPC/MDRI relief (Table 1 and Figure 1). ${ }^{3}$ The NPV of debt-to-GDP ratio falls to 22 percent in 2008 - declining 3 percentage points from the predicted level at completion point as a result of delayed loan disbursements. New borrowing associated with increased investment raises the NPV of debt-to-GDP through 2012 before it declines as investment levels off and growth is sustained. The NPV of debt-to-revenue and the debt service ratios fall considerably beneath their respective thresholds. While they too increase through 2012, they remain at comfortable levels throughout the projection period.

\section{The NPV of debt-to-exports ratio breaches the debt-burden threshold for a} protracted period. The NPV of debt-to-export ratio declined significantly to 114 percent in 2007 following full impact of HIPC and MDRI, but this is still above the indicative policydependent threshold of 100 percent. Furthermore, this ratio increases through 2012 due to new borrowing, and it stays above the threshold for a protracted period. It gradually gravitates towards the threshold over the medium term, due to sustained growth in receipts from tourism, re-export services, and agricultural exports.

Policy Dependent Debt Burden Thresholds under the Debt Sustainability Framework (Applying to external public debt)

\begin{tabular}{ccr}
\hline & Indicative thresholds 1/ & 2007 \\
\hline NPV of external debt & 30 & 24 \\
In percent of GDP & 100 & 114 \\
In percent of exports & 200 & 111 \\
In percent of revenues & & \\
Debt service & 15 & 20 \\
In percent of exports & 25 & 19 \\
In percent of revenues &
\end{tabular}

1/ The latest World Bank Country Policy and Institutional Assessment (CPIA) rates The Gambia a "poor performer".

\footnotetext{
${ }^{3}$ As outlined in the HIPC completion point DSA, outstanding debt at end-2007 before completion point is estimated at NPV US\$439 million. Following completion point, HIPC assistance reduces the NPV of existing debt by US\$92 million while MDRI results in an additional US\$182.1 million reduction.
} 


\section{B. Alternative Scenarios and Stress Tests}

\section{Alternative scenarios reveal that external debt indicators are vulnerable to substantial deterioration under a range of scenarios (Table 2, Figure 1).}

- Under the "less favorable terms" scenario, the NPV of debt-to-GDP deteriorates above its threshold emphasizing the need for the authorities to refrain from non-concessional borrowing. With the exception of the debt service indicators, which remain beneath their thresholds, the debt stock indicators all deteriorate significantly under this scenario.

- If the US dollar GDP deflator is below its historical average, the debt-to-GDP, debt-toexports, and debt-to-revenues ratios all deteriorate significantly.

- A combination of adverse economic shocks (i.e. lower growth and exports) would also result in a significant rise in debt ratios.

- Table 2 (line B6) demonstrates the deterioration in the debt-to-GDP ratio as a result of an exchange rate depreciation. In particular, a one-time 30 percent depreciation results in the debt-to-GDP ratio breaching the 30 percent threshold for much of the projection period. This scenario serves to illustrate the importance of recent exchange rate appreciation on the baseline debt-to-GDP ratio. If this appreciation is not permanent, debt-to-GDP will rise above the indicative threshold in the near term despite HIPC and MDRI relief.

\section{Public Debt Sustainability}

\section{Baseline}

6. Domestic debt is expected to fall from 30.7 percent of GDP at the end of 2007 to 13.8 percent of GDP in 2012 and to 9.5 percent of GDP in 2027, reflecting sustained good fiscal performance. Recently fiscal performance has been aided by reforms to tax administration that are expected to maintain revenues close to 21 percent of GDP. Relatively restrained fiscal policy, as programmed for the medium term, should help lower domestic interest rates. Over the long term, the delivery of external debt relief and lower domestic interest rates should provide fiscal space to increase basic primary expenditures ${ }^{4}$ and offset a decline in externally-financed projects as a percent of GDP.

7. The NPV of public debt is projected to decline from about 56.0 percent of GDP in 2007 to 42.3 percent in 2012 and to 29.9 percent in 2027 (Table 3 and Figure 2). The biggest factor in the near term is a fall in the domestic debt. As a ratio of domestic revenues,

\footnotetext{
${ }^{4}$ Defined as expenditures excluding interest payments and externally-financed projects.
} 
the NPV of public debt is projected to fall from about 257 percent in 2007 to 141 percent at the end of the projection period.

\section{Alternative Scenarios and Stress Tests}

8. Stress tests indicate that public debt ratios are sensitive to a depreciation in the near term and a shock to GDP growth in the long term, but not to most other adverse shocks (Table 4 and Figure 2).

- Under a permanently lower output growth rate (4.3 percent instead of 5 percent), the NPV of total debt-to-GDP ratio would decline from 56 percent in 2007 to 48 percent in 2027, as opposed to declining to 30 percent under the baseline scenario. Similarly, the NPV of debt-to-revenues would be 219 percent in 2027 compared to 136 under the baseline and the debt service-to-revenue ratio would increase from 11 to 19 percent of GDP.

- With a one-time 30 percent depreciation in 2008, the debt-to-GDP ratio would rise from 50 percent to 63 percent and the debt-to-revenue ratio would rise from 217 percent to 271 percent in 2008 compared with the baseline. However, the effects would be mitigated over time and these ratios would not be significantly worse than under the baseline in 2027.

- In contrast, the outlook for public debt is not sensitive to shocks to the primary balance, a combination of shocks to growth and the primary balance, or a ten percent increase in other debt-creating flows. Under none of these scenarios does the debt-to-GDP or debtto-revenue ratio rise much above the baseline in 2008-2009 or out to 2027.

- $\quad$ Because the primary balance was close to balance in 2007 , the alternative scenario based on the primary balance being unchanged would result in a rapid decline in The Gambia's public debt ratios with all debt eliminated by 2020 .

\section{Conclusion}

\section{The Gambia is at high risk of debt distress based on external debt burden}

indicators. The Gambia's debt situation has improved since the last DSA due to an improvement in the overall fiscal balance in 2007 and a decline in new borrowing. But given continuing risks, it will be important for the authorities' to finalize and implement the planned national debt strategy as soon as possible. Staff recommend that new borrowing be on highly concessional terms and that the authorities exercise restraint in contracting new loans. The major risks to debt sustainability include lower than expected economic and/or export growth, higher than expected new borrowing, or a deterioration in fiscal performance. 
Table 1. The Gambia: External Debt Sustainability Framework, HIPC and MDRI Scenario, 2007-2027 1/ In percent of GDP, unless

\begin{tabular}{|c|c|c|c|c|c|c|c|c|c|c|c|c|c|c|c|c|c|c|c|c|c|c|c|}
\hline & \multirow[b]{2}{*}{1996} & \multirow[b]{2}{*}{1997} & \multirow[b]{2}{*}{1998} & \multirow[b]{2}{*}{1999} & \multirow[b]{2}{*}{2000} & \multicolumn{3}{|c|}{ Actual } & \multirow[b]{2}{*}{2004} & \multirow[b]{2}{*}{2005} & \multirow[b]{2}{*}{2006} & \multirow{2}{*}{$\begin{array}{l}\text { Historical } \\
\text { Average } 66\end{array}$} & \multirow{2}{*}{$\begin{array}{l}\text { Standard } \\
\text { Deviation 6/ }\end{array}$} & \multirow{2}{*}{$\begin{array}{r}\text { Actual } \\
2007 \\
\end{array}$} & \multicolumn{5}{|c|}{ Projections } & \multirow[b]{2}{*}{$\begin{array}{l}2007-12 \\
\text { Average }\end{array}$} & \multirow[b]{2}{*}{2017} & \multirow[b]{2}{*}{2027} & \\
\hline & & & & & & 2001 & 2002 & 2003 & & & & & & & 2008 & 2009 & 2010 & 2011 & 2012 & & & & $\begin{array}{l}{ }_{\text {Average }}^{201327} \\
\text { A }\end{array}$ \\
\hline External debt (nominal) 1/ & $\ldots$ & 101.8 & 102.5 & 103.0 & 110.6 & 117.3 & 143.0 & 157.3 & 146.5 & 134.7 & 133.6 & & & 46.5 & 41.8 & 43.1 & 44.3 & 44.3 & 43.8 & & 39.2 & 30.4 & \\
\hline $\mathrm{o} / \mathrm{w}$ public and publicly guaranteed $(\mathrm{PPG})$ & $\ldots$ & 101.8 & 102.5 & 103.0 & 110.6 & 117.3 & 143.0 & 157.3 & 146.5 & 134.7 & 133.6 & & & 46.5 & 41.8 & 43.1 & 44.3 & 44.3 & 43.8 & & 39.2 & 30.4 & \\
\hline Change in external debt & $\ldots$ & $\ldots$ & 0.7 & 0.5 & 7.6 & 6.7 & 25.7 & 14.3 & -10.8 & -11.8 & -1.1 & & & -87.0 & -4.7 & 1.3 & 1.2 & 0.0 & -0.5 & & -0.8 & -0.9 & \\
\hline Identified net debt-creating flows & $\ldots$ & $\ldots$ & -4.0 & 0.1 & 2.1 & 0.9 & 15.7 & 8.3 & -26.8 & -17.3 & -15.2 & & & -6.8 & 3.2 & 2.8 & 2.0 & 1.8 & 1.6 & & 1.4 & 1.1 & \\
\hline Non-interest current account deficit & $\ldots$ & -0.3 & 1.0 & 1.5 & 2.0 & 1.4 & 1.6 & 3.4 & 4.1 & 13.2 & 9.8 & 3.8 & 4.3 & 11.0 & 13.9 & 12.7 & 11.8 & 11.4 & 10.8 & & 9.1 & 6.3 & 8.7 \\
\hline Deficit in balance of goods and services & $\ldots$ & 5.7 & 7.4 & 5.8 & 8.2 & 5.2 & 8.4 & 7.5 & 21.0 & 22.7 & 17.3 & & & 16.4 & 19.0 & 19.3 & 19.3 & 18.8 & 18.4 & & 15.6 & 10.4 & \\
\hline Exports & $\ldots$ & 27.0 & 30.1 & 28.0 & 26.8 & 25.8 & 30.4 & 31.8 & 31.7 & 28.4 & 30.6 & & & 21.1 & 18.9 & 18.6 & 18.5 & 18.4 & 18.3 & & 18.2 & 18.8 & \\
\hline Imports & $\ldots$ & 32.7 & 37.5 & 33.8 & 35.0 & 31.0 & 38.8 & 39.3 & 52.7 & 51.1 & 47.9 & & & 37.5 & 37.9 & 37.9 & 37.9 & 37.2 & 36.7 & & 33.8 & 29.2 & \\
\hline $\begin{array}{l}\text { Net current transfers (negative }=\text { inflow) } \\
\text { o / w official }\end{array}$ & $\cdots$ & -7.7 & -9.3 & -7.8 & $\begin{array}{c}-9.9 \\
-74\end{array}$ & $\begin{array}{r}-8.3 \\
-7.5\end{array}$ & $\begin{array}{l}-11.6 \\
-106\end{array}$ & $\begin{array}{l}-9.6 \\
-8.5\end{array}$ & $\begin{array}{l}-23.6 \\
-8.5\end{array}$ & $\begin{array}{c}-17.1 \\
-5.1\end{array}$ & $\begin{array}{c}-15.2 \\
-3.2\end{array}$ & -12.0 & 5.1 & -11.2 & $\begin{array}{c}-10.5 \\
-20\end{array}$ & $\begin{array}{c}-11.3 \\
-3.1\end{array}$ & $\begin{array}{l}-11.7 \\
-37\end{array}$ & $\begin{array}{r}-11.1 \\
-3.5\end{array}$ & $\begin{array}{c}-10.7 \\
-33\end{array}$ & & $\begin{array}{l}-9.0 \\
-2.6\end{array}$ & $\begin{array}{c}-5.9 \\
-09\end{array}$ & -8.1 \\
\hline $\begin{array}{l}\text { o/wo official } \\
\text { Other current account flows (negative }=\text { net inflow) }\end{array}$ & $\cdots$ & 1.6 & 2.9 & 3.5 & $\begin{array}{r}-7.4 \\
3.7\end{array}$ & $\begin{array}{r}-7.5 \\
4.6\end{array}$ & $\begin{array}{r}-10.6 \\
4.7\end{array}$ & $\begin{array}{r}-8.5 \\
5.4\end{array}$ & $\begin{array}{r}-8.3 \\
6.7\end{array}$ & -3.1 .5 & $\begin{array}{l}-3.2 \\
7.8\end{array}$ & & & $\begin{array}{r}-1.2 \\
58\end{array}$ & -2.0 & $\begin{array}{r}-3.1 \\
4.7\end{array}$ & -3.7 & $\begin{array}{l}-3.5 \\
3 .\end{array}$ & -3.3 & & -2.6 & -0.9 & \\
\hline Net FDI (negative = inflow) & $\ldots$ & -3.8 & -3.7 & -0.1 & -3.8 & -2.4 & -2.5 & -3.6 & -14.1 & -13.3 & -14.6 & -7.8 & 5.5 & $\begin{aligned} 5.8 \\
-12.5\end{aligned}$ & $\begin{array}{r}5.3 \\
-9.0\end{array}$ & $\begin{array}{ll}4.9 . \\
-8.0\end{array}$ & $\begin{array}{c}4.1 \\
-7.8\end{array}$ & -7.6 & $\begin{array}{r}3.2 \\
-7.4\end{array}$ & & -6.2 & $\begin{array}{r}1.99 \\
-42\end{array}$ & -56 - $>0$ \\
\hline Endogenous debt dynamics $2 /$ & .... & $\ldots$ & -1.3 & -1.3 & 3.9 & 2.0 & 16.5 & 8.5 & -16.8 & -17.2 & -10.4 & & & -5.3 & -1.7 & -1.9 & -2.0 & -2.0 & -1.8 & & -1.5 & -1.0 & -3.0 \\
\hline Contribution from nominal interest rate & & $\ldots$ & 1.4 & 1.4 & 1.2 & 1.2 & 1.2 & 1.7 & 2.0 & 1.9 & 1.7 & & & 1.4 & 0.4 & 0.4 & 0.4 & 0.4 & 0.4 & & 0.4 & 0.4 & \\
\hline Contribution from real GDP & $\ldots$ & $\ldots$ & -6.4 & -6.4 & -5.8 & -6.4 & 4.3 & -10.3 & -9.7 & -6.4 & -8.0 & & & -6.8 & -2.1 & -2.3 & -2.4 & -2.5 & -2.3 & & -1.9 & -1.5 & \\
\hline Contribution from price and exchange rate changes & & ... & 3.8 & 3.8 & 8.5 & 7.2 & 11.0 & 17.1 & -9.1 & -12.7 & -4.1 & & & & & & & & & & & & \\
\hline Residual (3-4) 3/ & 0.0 & ... & 4.7 & 0.4 & 5.5 & 5.8 & 10.0 & 6.0 & 16.0 & 5.5 & 14.0 & & & -80.2 & -7.9 & -1.5 & -0.8 & -1.8 & -2.1 & & -2.2 & -2.0 & \\
\hline $\mathrm{o} / \mathrm{w}$ exceptional financing & $\ldots$ & $\ldots$ & 0.0 & 0.0 & 0.0 & 0.0 & -0.1 & 0.0 & -0.4 & -0.3 & -0.3 & & & -0.3 & -0.8 & -0.7 & -0.6 & -0.4 & -0.4 & & -0.3 & -0.1 & \\
\hline NPV of external debt $4 /$ & $\ldots$ & $\ldots$ & $\ldots$ & $\ldots$ & ... & $\ldots$ & $\ldots$ & ... & $\ldots$ & $\ldots$ & 31.2 & & & 24.1 & 22.2 & 23.5 & 24.7 & 25.1 & 25.2 & & 24.0 & 20.0 & \\
\hline In percent of exports & $\ldots$ & $\ldots$ & $\ldots$ & $\ldots$ & $\ldots$ & $\ldots$ & $\ldots$ & $\ldots$ & $\ldots$ & $\ldots$ & 101.9 & & & 113.9 & 117.6 & 126.4 & 133.3 & 136.8 & 138.0 & & 131.3 & 106.4 & \\
\hline NPV of PPG external debt & $\ldots$ & ... & $\ldots$ & $\ldots$ & ... & $\ldots$ & $\ldots$ & $\ldots$ & $\ldots$ & $\ldots$ & 31.2 & & & 24.1 & 22.2 & 23.5 & 24.7 & 25.1 & 25.2 & & 24.0 & 20.0 & \\
\hline In percent of exports & $\ldots$ & $\ldots$ & ... & $\ldots$ & $\ldots$ & $\ldots$ & ... & $\ldots$ & $\ldots$ & $\ldots$ & 101.9 & & & 113.9 & 117.6 & 126.4 & 133.3 & 136.8 & 138.0 & & 131.3 & 106.4 & \\
\hline In percent of government revenues & ... & $\ldots$ & $\ldots$ & $\ldots$ & $\ldots$ & $\ldots$ & $\ldots$ & ... & & & 147.1 & & & 111.0 & 104.6 & 111.1 & 116.9 & 119.2 & 119.9 & & 116.5 & 96.3 & \\
\hline Debt service-to-exports ratio (in percent) & ... & 18.0 & 16.0 & $\ldots$ & 19.6 & 16.8 & 23.9 & 11.1 & 17.7 & 17.7 & 16.5 & & & 19.5 & 8.0 & 7.8 & 7.2 & 8.2 & 8.5 & & 9.2 & 8.4 & \\
\hline PPG debt service-to-exports ratio (in percent) & $\ldots$ & 18.0 & 16.0 & $\ldots$ & 19.6 & 16.8 & 23.9 & 11.1 & 17.7 & 17.7 & 16.5 & & & 19.5 & 8.0 & 7.8 & 7.2 & 8.2 & 8.5 & & 9.2 & 8.4 & \\
\hline PPG debt service-to-revenue ratio (in percent) & ... & 25.4 & 25.9 & ... & 28.4 & 28.8 & 44.5 & 22.4 & 26.9 & 25.5 & 23.8 & & & 19.0 & 7.1 & 6.9 & 6.3 & 7.2 & 7.4 & & 8.2 & 7.6 & \\
\hline Total gross financing need (millions of U.S. dollars) & $\ldots$ & $\ldots$ & & & 14.5 & 13.9 & 23.6 & 11.7 & -17.6 & 22.8 & 1.2 & & & 16.6 & 50.1 & 51.8 & 48.8 & 53.0 & 53.7 & & 69.0 & 111.5 & \\
\hline Non-interest current account deficit that stabilizes debt ratio & $\ldots$ & & 0.3 & 0.9 & -5.7 & -5.3 & -24.1 & -10.9 & 14.9 & 25.0 & 11.0 & & & 98.1 & 18.6 & 11.4 & 10.6 & 11.4 & 11.3 & & 9.8 & 7.3 & \\
\hline Key macroeconomic assumptions & & & & & & & & & & & & & & & & & & & & & & & \\
\hline Real GDP growth (in percent) & & $\ldots$ & 6.5 & 6.4 & 5.5 & 5.8 & -3.2 & 6.9 & 7.0 & 5.1 & 6.5 & & 3.2 & 6.4 & 5.5 & 6.0 & 6.0 & 6.0 & 5.5 & 5.9 & 5.0 & 5.0 & 5.0 \\
\hline (change in percent) & $\ldots$ & $\ldots$ & -3.6 & -3.5 & -7.7 & -6.1 & -8.6 & -10.7 & 6.1 & 9.5 & 3.1 & -2 & 7.0 & 19.3 & 14.8 & 2.0 & 2.2 & 2.3 & 2.2 & 7.2 & 2.0 & 2.0 & 2.0 \\
\hline Effecti & $\ldots$ & $\ldots$ & 1.4 & 1.4 & 1.1 & 1.1 & 0.9 & 1.1 & 1.5 & 1.5 & 1.4 & 1.3 & 0.2 & 1.4 & 1.1 & 1.1 & 1.0 & 1.0 & 1.0 & 1.1 & 1.0 & 1.5 & 1.2 \\
\hline Growth of exports of G\&S (US dollar term & $\ldots$ & $\ldots$ & 14.5 & -4.2 & -7.0 & -4.3 & 4.3 & -0.3 & 13.3 & 2.9 & 18.4 & 4.2 & 9.2 & -12.3 & 8.1 & 6.9 & 7.9 & 7.5 & 7.3 & 4.2 & 7.3 & 7.4 & 7.3. \\
\hline Growth of imports of G\&S (US dollar tc & $\ldots$ & $\ldots$ & 17.6 & -7.4 & 0.9 & -12.0 & 10.8 & -3.3 & 52.3 & 11.6 & 2.9 & 8.1 & 19.1 & -0.6 & 22.3 & 8.4 & 8.2 & 6.5 & 6.3 & 8.5 & 5.0 & 5.5 & 5.5 \\
\hline Grant element of new public sector bor & & & & & & & & & & & & & & 42.4 & 45.1 & 42.6 & 44.5 & 45.7 & 45.6 & 44.3 & 45.0 & 45.0 & 45.0 \\
\hline Aid flows (in millions of US dollars) $7 /$ & 0.0 & 19.7 & 22.4 & 18.5 & 25.5 & 33.3 & 51.4 & 35.1 & 50.6 & 41.5 & 44.2 & & & 31.4 & 51.7 & 73.0 & 86.0 & 79.7 & 77.4 & & 85.3 & 92.6 & \\
\hline o/w Grants & & 8.6 & 5.2 & 5.4 & 10.5 & 16.5 & 12.4 & 19.3 & 18.2 & 7.7 & 0.3 & & & 7.8 & 15.8 & 26.1 & 34.2 & 34.4 & 35.6 & & 39.1 & 26.1 & \\
\hline $\mathrm{o} / \mathrm{w}$ Concessional loans & 0.0 & 14.2 & $\ldots$ & $\ldots$ & $\ldots$ & $\ldots$ & $\ldots$ & $\ldots$ & 32.3 & 33.8 & 43.9 & & & 23.6 & 35.9 & 46.8 & 51.8 & 45.3 & 41.8 & & 46.2 & 66.5 & \\
\hline Grant-equivalent financing (in percent of GDP) 8/ & & $\ldots$ & $\ldots$ & & $\ldots$ & $\ldots$ & $\ldots$ & $\ldots$ & $\ldots$ & $\ldots$ & & & & 2.8 & 4.1 & 5.5 & 6.3 & 5.6 & 5.1 & & 4.0 & 1.9 & 3.4 \\
\hline Grant-equivalent financing (in percent of extermal financing) $8 /$ & $\ldots$ & $\ldots$ & $\ldots$ & $\ldots$ & $\ldots$ & $\ldots$ & $\ldots$ & & $\ldots$ & $\ldots$ & $\ldots$ & & & 56.7 & 61.9 & 63.2 & 66.6 & 69.1 & 70.6 & & 70.2 & 60.5 & 67.9 \\
\hline & & & & & & & & & & & & & & & & & & & & & & & \\
\hline $\begin{array}{l}\text { Nominal GDP (millions of US dollars) } \\
\text { (NPVt-NPVt-1)/GDPt-1 (in percent) }\end{array}$ & ... & 409.8 & 420.8 & 431.9 & 420.9 & 417.9 & 369.7 & 353.0 & 401.0 & 461.3 & 506.7 & & & $\begin{array}{r}643.5 \\
-0.6\end{array}$ & $\begin{array}{r}779.3 \\
2.8\end{array}$ & $\begin{array}{r}843.0 \\
3.3\end{array}$ & $\begin{array}{r}913.2 \\
3.2\end{array}$ & $\begin{array}{r}990.7 \\
2.6\end{array}$ & $\begin{array}{r}1068.5 \\
2.1\end{array}$ & 2.2 & $\begin{array}{r}1513.3 \\
1.5\end{array}$ & $\begin{array}{r}2993.3 \\
0.9\end{array}$ & 1.3 \\
\hline
\end{tabular}

1/Includes both public and private sector external debt.

1/ Includes both public and private sector external debt.
$2 /$ Derived as $[\mathrm{r}-\mathrm{g}-\rho(1+\mathrm{g}) /(1+\mathrm{g}+\rho+\mathrm{g} \rho)$ times previous period debt ratio, with $\mathrm{r}=$ nominal interest rate; $\mathrm{g}=$ real GDP growth rate, and $\rho=$ growth rate of GDP deflator in U.S. dollar terms.

$3 /$ Includes exceptional financing (i.e., changes in arrears and debt relief); changes in gross foreign assets; and valuation adjustments. For projections also includes contribution from price and exchange rate changes. 4/ Assumes that NPV of private sector debt is equivalent to its face value.
$5 /$ Current-year interest payments divided by previous period debt stock.

6/ Historical averages and standard deviations are generally derived over the past 10 years, subject to data availability.

8/ Grant-equivalent financing includes grants provided directly to the government and through new borrowing (difference between the face value and the NPV of new debt). 
Table 2. The Gambia: Sensitivity Analyses for Key Indicators of Public and Publicly Guaranteed External Debt, 2007-27 including HIPC and MDRI (In percent)

\begin{tabular}{|c|c|c|c|c|c|c|c|c|}
\hline & \multirow{2}{*}{$\begin{array}{c}\text { Actual } \\
2007\end{array}$} & \multicolumn{7}{|c|}{ Projections } \\
\hline & & 2008 & 2009 & 2010 & 2011 & 2012 & 2017 & 2027 \\
\hline \multicolumn{9}{|c|}{ NPV of debt-to-GDP ratio } \\
\hline Baseline & 24 & 22 & 24 & 25 & 25 & 25 & 24 & 20 \\
\hline \multicolumn{9}{|l|}{ A. Alternative Scenarios } \\
\hline A1. Key variables at their historical averages in 2008-27 1/ & 24 & 22 & 21 & 20 & 18 & 16 & 6 & -5 \\
\hline A2. New public sector loans on less favorable terms in 2008-27 2/ & 24 & 23 & 26 & 28 & 29 & 30 & 32 & 30 \\
\hline \multicolumn{9}{|l|}{ B. Bound Tests } \\
\hline B1. Real GDP growth at historical average minus one standard deviation in 2008-09 & 24 & 23 & 25 & 27 & 27 & 27 & 26 & 22 \\
\hline B2. Export value growth at historical average minus one standard deviation in 2008-09 3/ & 24 & 23 & 27 & 28 & 28 & 28 & 26 & 21 \\
\hline B3. US dollar GDP deflator at historical average minus one standard deviation in 2008-09 & 24 & 28 & 34 & 35 & 36 & 36 & 34 & 29 \\
\hline B4. Net non-debt creating flows at historical average minus one standard deviation in 2008-09 4/ & 24 & 29 & 36 & 37 & 37 & 36 & 32 & 23 \\
\hline B5. Combination of B1-B4 using one-half standard deviation shocks & 24 & 30 & 41 & 42 & 42 & 42 & 38 & 29 \\
\hline B6. One-time 30 percent nominal depreciation relative to the baseline in 20085 / & 24 & 30 & 32 & 33 & 34 & 34 & 32 & 27 \\
\hline \multicolumn{9}{|c|}{ NPV of debt-to-exports ratio } \\
\hline Baseline & 114 & 118 & 126 & 133 & 137 & 138 & 131 & 106 \\
\hline \multicolumn{9}{|l|}{ A. Alternative Scenarios } \\
\hline A1. Key variables at their historical averages in 2007-26 1/ & 114 & 117 & 112 & 108 & 100 & 90 & 34 & -25 \\
\hline A2. New public sector loans on less favorable terms in $2007-262 /$ & 114 & 123 & 137 & 151 & 159 & 164 & 173 & 160 \\
\hline \multicolumn{9}{|l|}{ B. Bound Tests } \\
\hline B1. Real GDP growth at historical average minus one standard deviation in 2008-09 & 114 & 118 & 126 & 133 & 137 & 138 & 131 & 106 \\
\hline B2. Export value growth at historical average minus one standard deviation in 2008-09 3/ & 114 & 141 & 186 & 194 & 197 & 198 & 184 & 142 \\
\hline B3. US dollar GDP deflator at historical average minus one standard deviation in 2008-09 & 114 & 118 & 126 & 133 & 137 & 138 & 131 & 106 \\
\hline B4. Net non-debt creating flows at historical average minus one standard deviation in 2008-09 4/ & 114 & 152 & 193 & 198 & 199 & 199 & 174 & 121 \\
\hline B5. Combination of B1-B4 using one-half standard deviation shocks & 114 & 141 & 184 & 191 & 194 & 194 & 177 & 132 \\
\hline B6. One-time 30 percent nominal depreciation relative to the baseline in 2008 5/ & 114 & 118 & 126 & 133 & 137 & 138 & 131 & 106 \\
\hline \multicolumn{9}{|c|}{ NPV of debt-to-revenue ratio } \\
\hline Baseline & 111 & 105 & 111 & 117 & 119 & 120 & 117 & 96 \\
\hline \multicolumn{9}{|l|}{ A. Alternative Scenarios } \\
\hline A1. Key variables at their historical averages in $2007-261$ / & 111 & 104 & 99 & 94 & 87 & 78 & 30 & -23 \\
\hline A2. New public sector loans on less favorable terms in 2007-26 2/ & 111 & 109 & 121 & 132 & 139 & 143 & 154 & 145 \\
\hline \multicolumn{9}{|l|}{ B. Bound Tests } \\
\hline B1. Real GDP growth at historical average minus one standard deviation in 2008-09 & 111 & 108 & 120 & 126 & 128 & 129 & 125 & 104 \\
\hline B2. Export value growth at historical average minus one standard deviation in 2008-09 3/ & 111 & 111 & 127 & 133 & 134 & 135 & 127 & 100 \\
\hline B3. US dollar GDP deflator at historical average minus one standard deviation in 2008-09 & 111 & 133 & 159 & 167 & 170 & 171 & 166 & 137 \\
\hline B4. Net non-debt creating flows at historical average minus one standard deviation in 2008-09 4/ & 111 & 135 & 170 & 173 & 174 & 173 & 155 & 110 \\
\hline B5. Combination of B1-B4 using one-half standard deviation shocks & 111 & 143 & 192 & 198 & 200 & 200 & 186 & 142 \\
\hline B6. One-time 30 percent nominal depreciation relative to the baseline in 20085 / & 111 & 142 & 150 & 158 & 161 & 162 & 158 & 130 \\
\hline
\end{tabular}


Table 2. The Gambia: Sensitivity Analyses for Key Indicators of Public and Publicly Guaranteed External Debt, 2007-27 (continued) with HIPC and MDRI (In percent)

Debt service-to-exports ratio

Baseline

A. Alternative Scenarios

A1. Key variables at their historical averages in 2008-27 1/

A2. New public sector loans on less favorable terms in 2008-27 2/

B. Bound Tests

B1. Real GDP growth at historical average minus one standard deviation in 2008-09

B2. Export value growth at historical average minus one standard deviation in 2008-09 3/

B3. US dollar GDP deflator at historical average minus one standard deviation in 2008-09

B4. Net non-debt creating flows at historical average minus one standard deviation in 2008-09 4

B5. Combination of B1-B4 using one-half standard deviation shocks

B6. One-time 30 percent nominal depreciation relative to the baseline in 20085 /

$\begin{array}{rrrrrrrr}19 & 8 & 8 & 7 & 8 & 9 & \mathbf{9} & 8 \\ & & & & & & & \\ 20 & 10 & 9 & 10 & 10 & 11 & \mathbf{7} & 0 \\ 20 & 8 & 8 & 9 & 10 & 10 & \mathbf{1 1} & 11 \\ & & & & & & & \\ & & & & & & & \\ 20 & 8 & 8 & 8 & 8 & 9 & \mathbf{8} & 7 \\ 20 & 9 & 10 & 11 & 11 & 11 & \mathbf{1 2} & 10 \\ 20 & 8 & 8 & 8 & 8 & 9 & \mathbf{8} & 7 \\ 20 & 8 & 8 & 9 & 9 & 10 & \mathbf{1 2} & 9 \\ 20 & 9 & 9 & 10 & 10 & 11 & \mathbf{1 2} & 9 \\ 20 & 8 & 8 & 8 & 8 & 9 & \mathbf{8} & 7\end{array}$

Debt service-to-revenue ratio

Baseline

A. Alternative Scenario

A1. Key variables at their historical averages in 2008-27 1/

A2. New public sector loans on less favorable terms in 2008-27 2/

B. Bound Tests

B1. Real GDP growth at historical average minus one standard deviation in 2008-09

B2. Export value growth at historical average minus one standard deviation in 2008-09 3/

B3. US dollar GDP deflator at historical average minus one standard deviation in 2008-09

B4. Net non-debt creating flows at historical average minus one standard deviation in 2008-09 4/

B5. Combination of B1-B4 using one-half standard deviation shocks

B6. One-time 30 percent nominal depreciation relative to the baseline in $20085 /$

Memorandum item:

Grant element assumed on residual financing (i.e., financing required above baseline) $6 /$

Source: Staff projections and simulations.

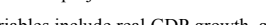

growth of GDP deflator (in U.S. dollar terms), non-interest current account in percent of GDP, and non-debt creating flows.

$2 /$ Assumes that the interest rate on new borrowing is by 2 percentage points higher than in the baseline., while grace and maturity periods are the same as in the baseline

3/ Exports values are assumed to remain permanently at the lower level, but the current account as a share of GDP is assumed to return to its baseline level after the shock (implicitly assuming

an offsetting adjustment in import levels).

4/ Includes official and private transfers and FD

5/ Depreciation is defined as percentage decline in dollar/local currency rate, such that it never exceeds 100 percent.

6/ Applies to all stress scenarios except for A2 (less favorable financing) in which the terms on all new financing are as specified in footnote 2. 
Table 3.The Gambia: Public Sector Debt Sustainability Framework, Baseline Scenario, 2001-2027 (In percent of GDP, unless otherwise indicated)

\begin{tabular}{|c|c|c|c|c|c|c|c|c|c|c|c|c|c|c|c|c|c|c|}
\hline & \multicolumn{6}{|c|}{ Actual } & \multirow[b]{2}{*}{$\begin{array}{l}\text { Historical } \\
\text { Average 5/ }\end{array}$} & \multirow[b]{2}{*}{$\begin{array}{c}\text { Standard } \\
\text { Deviation 5/ }\end{array}$} & \multicolumn{5}{|l|}{ Actual } & \multicolumn{5}{|c|}{ Projections } \\
\hline & 2001 & 2002 & 2003 & 2004 & 2005 & 2006 & & & 2007 & 2008 & 2009 & 2010 & 2011 & 2012 & $\begin{array}{l}2007-12 \\
\text { Average }\end{array}$ & 2017 & 2027 & $\begin{array}{l}2013-27 \\
\text { Average }\end{array}$ \\
\hline $\begin{array}{l}\text { Public sector debt } 1 / \\
\text { o/w foreign-currency denominated }\end{array}$ & $\begin{array}{l}155.4 \\
117.3\end{array}$ & $\begin{array}{l}179.6 \\
143.0\end{array}$ & $\begin{array}{l}185.2 \\
157.3\end{array}$ & $\begin{array}{l}179.4 \\
146.5\end{array}$ & $\begin{array}{l}170.2 \\
134.7\end{array}$ & $\begin{array}{l}167.8 \\
133.3\end{array}$ & & & $\begin{array}{l}77.2 \\
46.5\end{array}$ & $\begin{array}{l}67.5 \\
41.8\end{array}$ & $\begin{array}{l}65.5 \\
43.1\end{array}$ & $\begin{array}{l}63.4 \\
44.3\end{array}$ & $\begin{array}{l}60.3 \\
44.3\end{array}$ & $\begin{array}{l}57.6 \\
43.8\end{array}$ & & $\begin{array}{l}50.3 \\
39.2\end{array}$ & $\begin{array}{l}39.9 \\
30.4\end{array}$ & \\
\hline Change in public sector debt & 13.3 & 24.2 & 5.6 & -5.8 & -9.2 & -2.3 & & & -90.6 & -9.7 & -2.0 & -2.1 & -3.1 & -2.7 & & -0.8 & -1.2 & \\
\hline Identified debt-creating flows & -14.0 & 27.4 & -8.5 & -32.1 & -14.4 & -5.0 & & & -43.5 & -6.9 & -5.2 & -5.0 & -5.1 & -4.6 & & -1.5 & -2.4 & \\
\hline Primary deficit & 9.4 & 1.5 & 0.0 & -1.3 & 0.3 & 0.2 & 0.6 & 3.4 & -5.7 & -1.2 & 0.5 & 0.6 & -0.2 & 0.0 & -1.0 & 1.7 & -0.2 & 0.8 \\
\hline Revenue and grants & 16.1 & 19.1 & 17.0 & 25.5 & 21.4 & 22.5 & & & 22.9 & 23.1 & 24.2 & 24.8 & 24.5 & 24.3 & & 23.7 & 22.0 & \\
\hline of which: grants & 1.0 & 2.8 & 1.3 & 3.3 & 1.5 & 1.3 & & & 1.1 & 1.8 & 3.0 & 3.7 & 3.4 & 3.3 & & 2.6 & 0.9 & \\
\hline Primary (noninterest) expenditure & 25.6 & 20.6 & 17.0 & 24.1 & 21.7 & 22.6 & & & 17.1 & 22.0 & 24.7 & 25.4 & 24.3 & 24.3 & & 25.4 & 21.8 & \\
\hline Automatic debt dynamics & -23.4 & 28.3 & -7.1 & -29.3 & -14.0 & -6.7 & & & -36.9 & -3.0 & -3.2 & -3.4 & -3.2 & -3.0 & & -2.2 & -1.7 & \\
\hline Contribution from interest rate/growth differential & -24.1 & 3.5 & -14.3 & -10.6 & -4.6 & -5.9 & & & -8.4 & -2.6 & -2.9 & -3.1 & -3.1 & -2.8 & & -2.2 & -1.7 & \\
\hline of which: contribution from average real interest rate & -1.3 & -1.7 & -2.8 & 1.5 & 4.2 & 4.6 & & & 1.6 & 1.4 & 0.9 & 0.6 & 0.4 & 0.3 & & 0.2 & 0.3 & \\
\hline of which: contribution from real GDP growth & -7.7 & 5.2 & -11.5 & -12.2 & -8.7 & -10.5 & & & -10.0 & -4.0 & -3.8 & -3.7 & -3.6 & -3.1 & & -2.4 & -2.0 & \\
\hline Contribution from real exchange rate depreciation & 0.7 & 24.9 & 7.2 & -18.7 & -9.4 & -0.8 & & & -28.6 & -0.4 & -0.3 & -0.2 & -0.1 & -0.1 & & $\ldots$ & $\ldots$ & \\
\hline Other identified debt-creating flows & 0.0 & -2.5 & -1.4 & -1.5 & -0.7 & 1.5 & & & -0.8 & -2.7 & -2.4 & -2.3 & -1.8 & -1.6 & & -1.0 & -0.4 & \\
\hline Privatization receipts (negative) & 0.0 & 0.0 & 0.0 & 0.0 & -0.4 & 0.0 & & & -0.1 & -0.1 & 0.0 & 0.0 & 0.0 & 0.0 & & 0.0 & 0.0 & \\
\hline Recognition of implicit or contingent liabilities & 0.0 & 0.0 & 0.0 & 0.0 & 0.0 & 1.5 & & & 0.0 & 0.0 & 0.0 & 0.0 & 0.0 & 0.0 & & 0.0 & 0.0 & \\
\hline Debt relief (HIPC and other) & -1.0 & -2.0 & -1.1 & -1.3 & -0.1 & 0.0 & & & -0.6 & -2.6 & -2.4 & -2.3 & -1.8 & -1.6 & & -1.0 & -0.4 & \\
\hline Other (specify, e.g. bank recapitalization) & 1.0 & -0.4 & -0.2 & -0.2 & -0.2 & 0.0 & & & 0.0 & 0.0 & 0.0 & 0.0 & 0.0 & 0.0 & & 0.0 & 0.0 & \\
\hline Residual, including asset changes & 27.3 & -3.2 & 14.1 & 26.3 & 5.2 & 2.7 & & & -47.2 & -2.8 & 3.2 & 2.9 & 2.0 & 1.9 & & 0.7 & 1.1 & \\
\hline NPV of public sector debt & 38.1 & 36.6 & 27.9 & 32.9 & 35.5 & 65.7 & & & 56.0 & 50.3 & 49.2 & 47.3 & 44.6 & 42.3 & & 37.0 & 29.9 & \\
\hline $\mathrm{o} / \mathrm{w}$ foreign-currency denominated & 0.0 & 0.0 & 0.0 & 0.0 & 0.0 & 31.2 & & & 25.3 & 24.6 & 26.7 & 28.2 & 28.6 & 28.5 & & 25.9 & 20.3 & \\
\hline $\mathrm{o} / \mathrm{w}$ external & $\ldots$ & $\ldots$ & $\ldots$ & $\ldots$ & $\ldots$ & 31.2 & & & 25.3 & 24.6 & 26.7 & 28.2 & 28.6 & 28.5 & & 25.9 & 20.3 & \\
\hline $\mathrm{NPV}$ of contingent liabilities (not included in public sector debt) & & & & $\ldots$ & & & & & 0.0 & 0.0 & 0.0 & 0.0 & 0.0 & 0.0 & & 0.0 & 0.0 & \\
\hline Gross financing need $2 /$ & 17.1 & 10.4 & 6.5 & 9.8 & 12.3 & 10.4 & & & 2.5 & 4.1 & 5.0 & 4.6 & 3.3 & 3.2 & & 4.4 & 2.1 & \\
\hline NPV of public sector debt-to-revenue and grants ratio (in percent) & 236.0 & 191.5 & 164.0 & 129.1 & 165.6 & 292.2 & & & 244.7 & 217.3 & 203.1 & 190.7 & 182.2 & 174.0 & & 156.1 & 135.7 & \\
\hline NPV of public sector debt-to-revenue ratio (in percent) & 252.2 & 224.2 & 177.8 & 148.2 & 178.4 & 309.6 & & & 256.9 & 236.0 & 231.5 & 223.8 & 211.7 & 201.1 & & 175.1 & 141.3 & \\
\hline o/w external 3/ & & & & & & 146.9 & & & 116.1 & 115.4 & 125.9 & 133.5 & 135.8 & 135.4 & & 122.5 & 96.3 & \\
\hline Debt service-to-revenue and grants ratio (in percent) $4 /$ & 475 & 57.9 & 464 & 424 & 54.6 & 436 & & & 339 & 202 & 160 & 132 & 129 & 120 & & 1114 & 106 & \\
\hline Debt service-to-revenue ratio (in percent) $4 /$ & 50.7 & 67.8 & 50.2 & 48.7 & 58.8 & 46.2 & & & 35.6 & 22.0 & 18.3 & 15.5 & 15.0 & 13.9 & & 12.8 & 11.0 & \\
\hline Primary deficit that stabilizes the deb-to-GDP ratio & & & & 4.5 & 9.5 & 2.5 & & & 84.9 & 8.5 & 2.5 & 2.7 & 2.9 & 2.7 & & 2.5 & 1.0 & \\
\hline Key macroeconomic and fiscal assumptions & & & & & & & & & & & & & & & & & & \\
\hline Real GDP growth (in percent) & 5.8 & -3.2 & 6.9 & 7.0 & 5.1 & 6.5 & 5.0 & 3.1 & 6.3 & 5.5 & 6.0 & 6.0 & 6.0 & 5.5 & 5.9 & 5.0 & 5.0 & 5.0 \\
\hline Average nominal interest rate on forex debt (in percent) & 1.3 & 1.2 & 1.6 & 1.6 & 1.4 & 1.4 & 1.4 & 0.1 & 1.2 & 1.0 & 1.1 & 1.0 & 1.1 & 1.1 & 1.1 & 1.0 & 1.5 & 1.2 \\
\hline Average real interest rate on domestic currency debt (in percent) & -2.1 & -4.2 & -8.7 & 8.9 & 17.3 & 12.9 & 7.4 & 9.2 & 5.9 & 5.3 & 4.8 & 4.7 & 4.8 & 4.9 & 5.1 & 5.8 & 4.1 & 5.6 \\
\hline Real exchange rate depreciation (in percent, + indicates depreciation) & 0.7 & 20.5 & 5.4 & -12.8 & -6.8 & -0.6 & 4.9 & 11.7 & -22.8 & & $\ldots$ & & & ... & $\ldots$ & $\ldots$ & $\ldots$ & $\ldots$ \\
\hline Inflation rate (GDP deflator, in percent) & 15.2 & 16.1 & 27.4 & 12.2 & 4.1 & 1.4 & 8.9 & 8.6 & 5.7 & 6.0 & 5.5 & 4.8 & 4.5 & 4.2 & 5.1 & 4.0 & 4.0 & 4.0 \\
\hline Growth of real primary spending (defflated by GDP deflator, in percent) & 47.8 & -22.1 & -11.6 & 51.6 & -5.5 & 11.2 & 5.5 & 25.6 & -19.5 & 35.3 & 19.0 & 9.1 & 1.5 & 5.6 & 8.5 & 3.0 & 3.5 & 4.3 \\
\hline Grant element of new external borrowing (in percent) & $\ldots$ & $\ldots$ & $\ldots$ & $\ldots$ & $\ldots$ & $\ldots$ & & & 42.6 & 43.2 & 43.3 & 44.1 & 45.0 & 45.0 & 43.9 & 45.0 & 45.0 & ( \\
\hline
\end{tabular}

1/[Indicate coverage of public sector, e.g.g., general government or nonfinancial public sector. Also whether net or gross debt is used.]

$2 /$ Gross financing need is defined as the primary deficit plus debt service plus the stock of short-term debt at the end of the last period

3/ Revenues excluding grants.

Derast 10 years, subject to data availability. 
Table 4.The Gambia: Sensitivity Analysis for Key Indicators of Public Debt 2007-2027

\begin{tabular}{ll}
\hline \\
\hline Baseline & NPV of Debt-to-GDP Ratio
\end{tabular}

Baseline

A. Alternative scenarios

A1. Real GDP growth and primary balance are at historical averages

A2. Primary balance is unchanged from 2007

A3. Permanently lower GDP growth 1 /

\section{B. Bound tests}

B1. Real GDP growth is at historical average minus one standard deviations in 2008-2009

B2. Primary balance is at historical average minus one standard deviations in 2008-2009

B3. Combination of B1-B2 using one half standard deviation shock

B4. One-time 30 percent real depreciation in 2008

B5. 10 percent of GDP increase in other debt-creating flows in 2008

NPV of Debt-to-Revenue Ratio 2/

Baseline

\section{A. Alternative scenarios}

A1. Real GDP growth and primary balance are at historical averages

A2. Primary balance is unchanged from 2007

A3. Permanently lower GDP growth $1 /$

\section{B. Bound tests}

B1. Real GDP growth is at historical average minus one standard deviations in 2008-2009

B2. Primary balance is at historical average minus one standard deviations in 2008-2009

B3. Combination of B1-B2 using one half standard deviation shocks

B4. One-time 30 percent real depreciation in 2008

B5. 10 percent of GDP increase in other debt-creating flows in 2008

\section{Projections}

$2008-200$

$\begin{array}{rrrrrrrr}56 & 50 & 49 & 47 & 45 & 42 & 37 & 30 \\ & & & & & & & \\ & & & & & & & \\ 56 & 52 & 52 & 50 & 49 & 47 & 38 & 32 \\ 56 & 48 & 43 & 38 & 33 & 28 & 6 & -21 \\ 56 & 51 & 51 & 50 & 47 & 46 & 44 & 48\end{array}$

$\begin{array}{llllllll}56 & 53 & 55 & 54 & 52 & 50 & 48 & 45 \\ 56 & 54 & 55 & 53 & 50 & 47 & 41 & 32 \\ 56 & 54 & 55 & 53 & 50 & 47 & 40 & 31 \\ 56 & 63 & 61 & 58 & 54 & 51 & 43 & 33 \\ 56 & 57 & 55 & 53 & 50 & 48 & 41 & 32\end{array}$

Debt Service-to-Revenue Ratio 2/

$\begin{array}{llllllll}245 & 217 & 203 & 191 & 182 & 174 & 156 & 136\end{array}$

$\begin{array}{rlllllll}245 & 225 & 213 & 202 & 197 & 191 & 159 & 143\end{array}$

$\begin{array}{rrrrrrrr}245 & 206 & 178 & 154 & 135 & 116 & 25 & -93 \\ 245 & 221 & 209 & 199 & 193 & 187 & 184 & 219\end{array}$

$\begin{array}{llllllll}245 & 228 & 226 & 216 & 211 & 205 & 199 & 203\end{array}$

$\begin{array}{llllllll}245 & 234 & 227 & 212 & 203 & 194 & 172 & 144\end{array}$

$\begin{array}{llllllll}245 & 233 & 227 & 212 & 203 & 193 & 169 & 142 \\ 245 & 271 & 251 & 233 & 221 & 210 & 180 & 150\end{array}$

$\begin{array}{llllllll}245 & 271 & 251 & 233 & 221 & 210 & 180 & 150 \\ 245 & 247 & 228 & 214 & 205 & 196 & 174 & 145\end{array}$

Baseline

$\begin{array}{llllllll}34 & 20 & 16 & 13 & 13 & 12 & 11 & 11\end{array}$

A. Alternative scenarios

A1. Real GDP growth and primary balance are at historical averages

A2. Primary balance is unchanged from 2007

A3. Permanently lower GDP growth 1/

$\begin{array}{rrrrrrrr}34 & 20 & 18 & 13 & 12 & 13 & 9 & 12 \\ 34 & 20 & 9 & 2 & 0 & 0 & -5 & -13 \\ 34 & 20 & 15 & 13 & 13 & 12 & 14 & 19\end{array}$

B. Bound tests

B1. Real GDP growth is at historical average minus one standard deviations in 2008-2009

B2. Primary balance is at historical average minus one standard deviations in 2008-2009

B3. Combination of B1-B2 using one half standard deviation shocks

B4. One-time 30 percent real depreciation in 2008

B5. 10 percent of GDP increase in other debt-creating flows in 2008

\begin{tabular}{|c|c|c|c|c|c|}
\hline 20 & 17 & 15 & 15 & 15 & 16 \\
\hline 20 & 22 & 19 & 14 & 12 & 12 \\
\hline 20 & 20 & 16 & 13 & 12 & 11 \\
\hline 20 & 16 & 13 & 13 & 12 & 12 \\
\hline 20 & 28 & 16 & 13 & 12 & 12 \\
\hline
\end{tabular}

Sources: Country authorities; and Fund staff estimates and projections.

1/ Assumes that real GDP growth is at baseline minus one standard deviation divided by the square root of 20 (i.e., the length of the projection period).

$2 /$ Revenues are defined inclusive of grants. 
Figure 1. The Gambia: Indicators of Public and Publicly Guaranteed External Debt Under Alternative Scenarios, 2007-2027
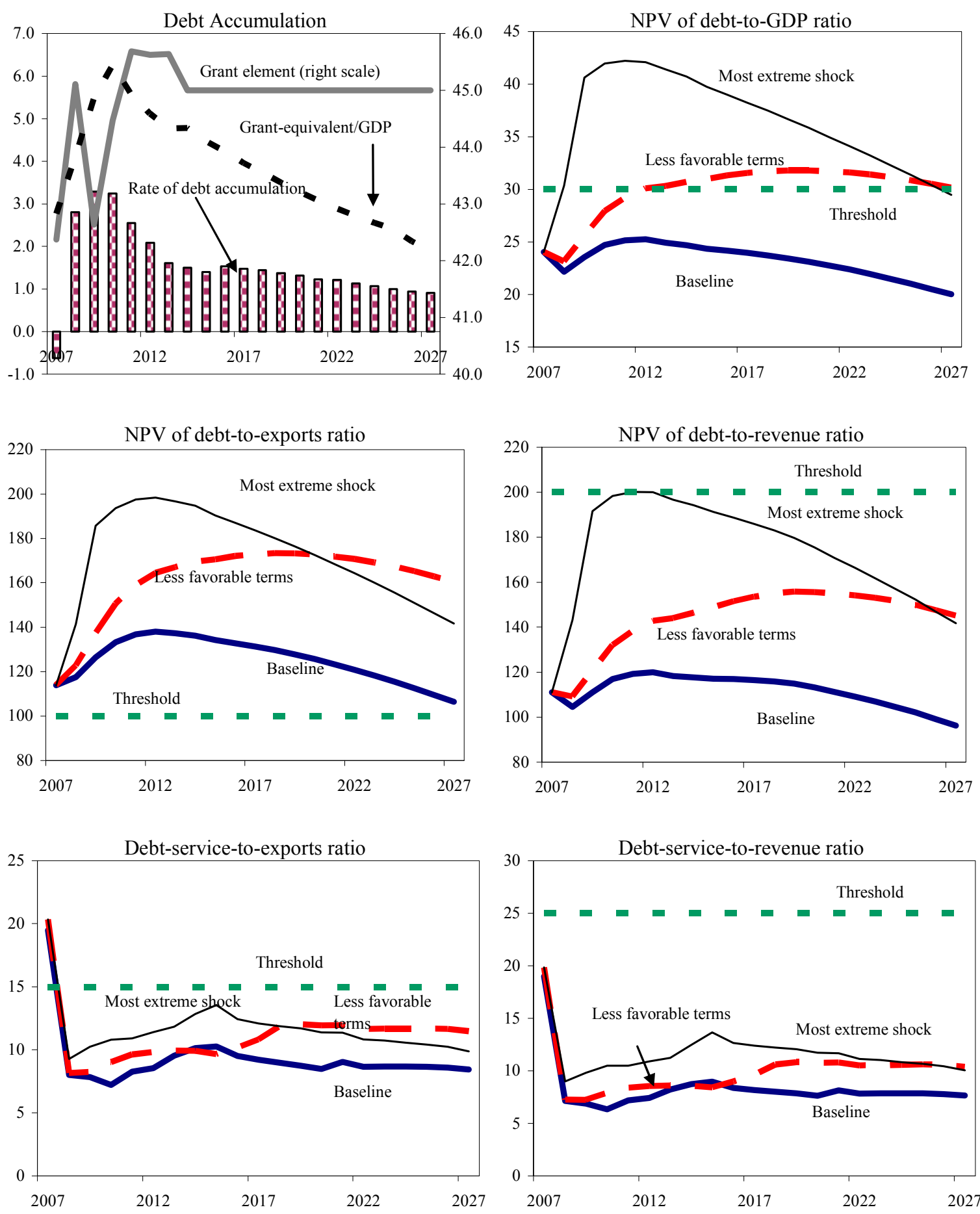

Source: Staff projections and simulations. 
Figure 2.The Gambia: Indicators of Public Debt Under Alternative Scenarios, 2007-2027 1/

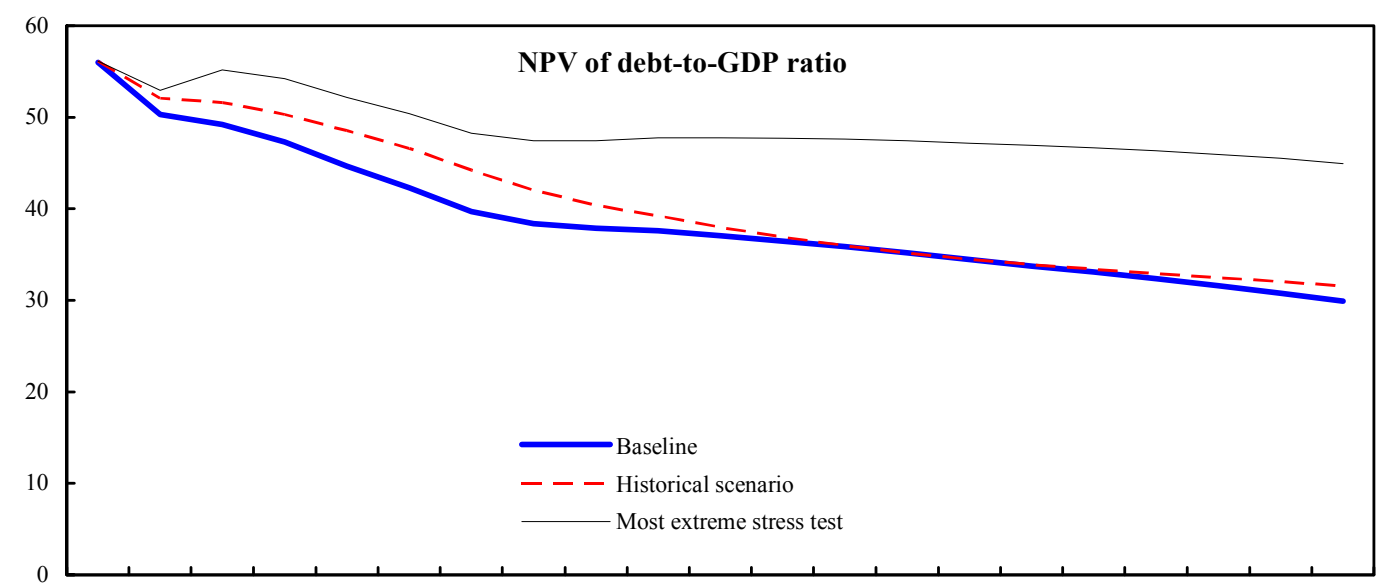

200720082009201020112012201320142015201620172018201920202021202220232024202520262027
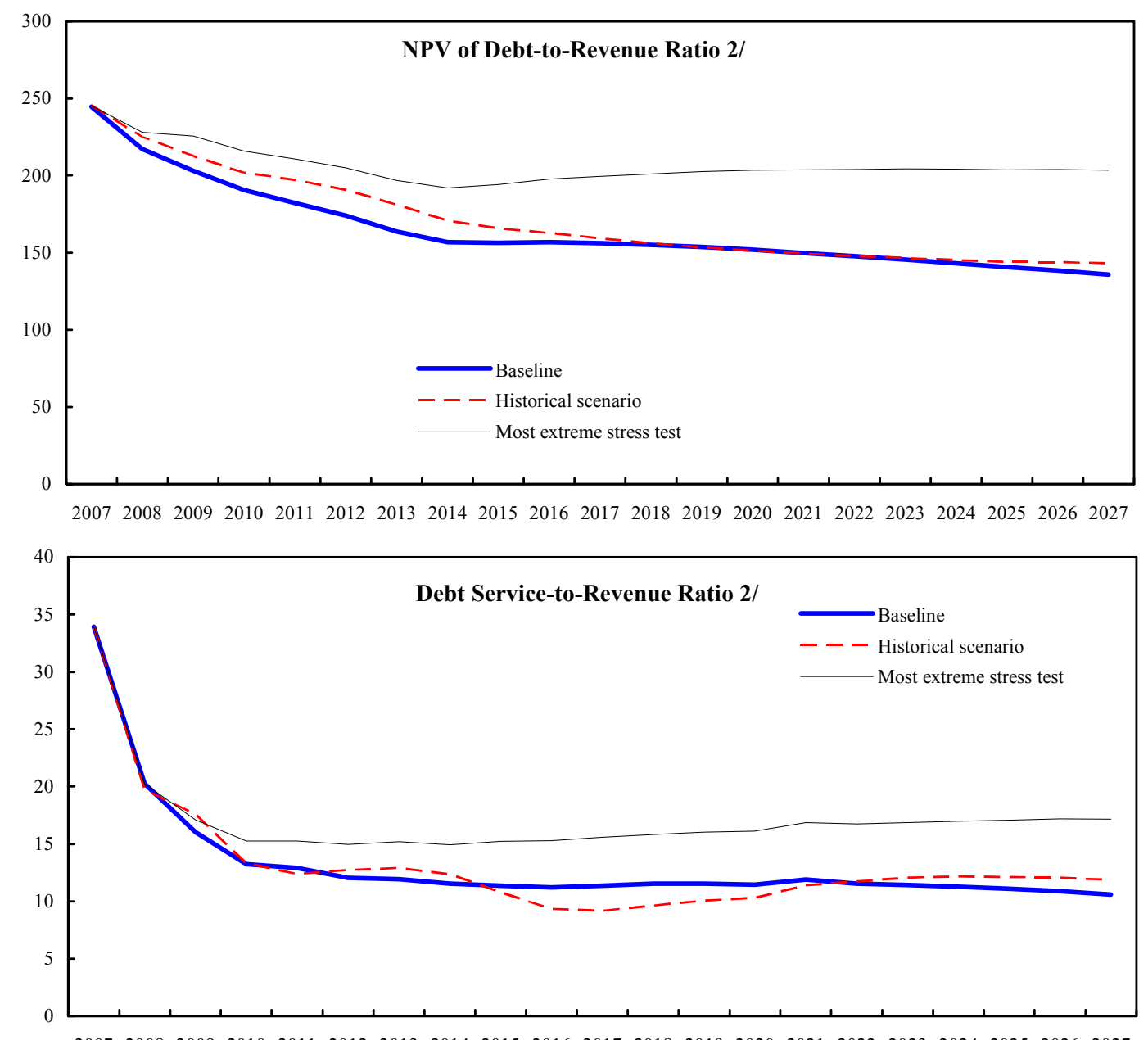

Source: Staff projections and simulations.

1/ Most extreme stress test is test that yields highest ratio in 2017.

2/ Revenue including grants. 


\section{INTERNATIONAL MONETARY FUND}

\section{THE GAMBIA}

\section{Staff Report for the 2008 Article IV Consultation, Third Review Under the Three-Year Arrangement Under the Poverty Reduction and Growth Facility, and Request for Waiver of Performance Criteria-Informational Annex}

Prepared by the African Department

(In consultation with other departments)

Approved by Hugh Bredenkamp and Mark Plant

August 20, 2008

- $\quad$ Relations with the Fund. Describes financial and technical assistance from the Fund and provides information on the safeguards assessment and exchange rate system. Outstanding purchases and loans amounted to SDR 6.00 million (19.29 percent of quota) at end-July 2008.

- Joint Bank-Fund Work Program. Lists the work program of the Bank and the Fund on The Gambia during August 2008-July 2009.

- $\quad$ Relations with the African Development Bank. Describes the African Development Bank Group program and portfolio.

- Statistical Issues. Assesses the quality of statistical data. The authorities have made progress in improving the compilation of economic and financial statistics. However, weaknesses in a broad range of economic statistics are hampering the analyses of economic developments in the country. 


\section{The Gambia: Relations with the Fund}

(As of July 31, 2008)

Membership status. Joined September 21, 1967. The Gambia accepted the obligations under Article VIII, Sections 2(a), 3, and 4, of the Fund's Articles of Agreements on January 21, 1993. It maintains an exchange system that is free of restrictions on the making of payments and transfers for current international transactions.

General Resources Account

Quota

Fund holdings of currency

Reserve position in Fund

SDR Department

Net cumulative allocation

Holdings

Outstanding Purchases and Loans

Poverty Reduction and Growth Facility (PRGF)

arrangements

\begin{tabular}{|c|c|}
\hline$\underline{\text { SDR Million }}$ & \% Quota \\
\hline 31.10 & 100.00 \\
\hline 29.62 & 95.23 \\
\hline 1.48 & 4.77 \\
\hline
\end{tabular}

SDR Million

5.12

0.05

$\underline{\text { SDR Million }}$

6.00

\section{Latest Financial Arrangements}

$\begin{array}{lcccc}\text { Type } & \text { Approval Date } & \text { Expiration Date } & \begin{array}{c}\text { Amount } \\ \text { Approved } \\ \text { (SDR Million) }\end{array} & \begin{array}{c}\text { Amount Drawn } \\ \text { (SDR Million) }\end{array} \\ \text { PRGF } & \text { Feb. 21, 2007 } & \text { Feb. 20, 2010 } & 14.00 & 6.00 \\ \text { PRGF } & \text { Jul. 18, 2002 } & \text { Jul. 17, 2005 } & 20.22 & 2.89 \\ \text { PRGF } & \text { Jun. 29, 1998 } & \text { Dec. 31, 2001 } & 20.61 & 20.61 \\ \text { PRGF } & \text { Nov. 23, 1988 } & \text { Nov. 25, 1991 } & 20.52 & 18.02\end{array}$

Projected Payments to Fund (SDR million; based on current use of resources and present holdings of SDRs) ${ }^{1}$

\begin{tabular}{lrrrrr} 
& \multicolumn{7}{c}{ Forthcoming } \\
\cline { 2 - 6 } Principal & $\underline{2008}$ & $\underline{2009}$ & $\underline{2010}$ & $\underline{2011}$ & $\underline{2012}$ \\
Charges/interest & 0 & 0 & 0 & 0 & 0.20 \\
Total & $\underline{0.09}$ & $\underline{0.19}$ & $\underline{0.19}$ & $\underline{0.19}$ & $\underline{0.19}$ \\
& & $\underline{0.19}$ & $\underline{0.19}$ & $\underline{0.19}$ & $\underline{0.39}$
\end{tabular}

\footnotetext{
${ }^{1}$ When a member has overdue financial obligations outstanding for more than three months, the amount of such arrears will be shown in this section.
} 


\section{Implementation of HIPC Initiative}

Enhanced

Framework

Commitment of HIPC assistance

Decision point date ${ }^{2}$

Dec. 11,2000

Assistance committed (yearend 2000 NPV terms) ${ }^{3}$

Total assistance (US\$ million)

66.60

Of which: IMF assistance (US\$ million)

2.30

SDR equivalent, million

1.80

Completion point date

December 19, 2007

Disbursement of IMF assistance (SDR million)

Assistance disbursed

Interim assistance

0.44

Completion point balance

1.36

Additional disbursement of interest income ${ }^{4}$

0.49

\section{Implementation of Multilateral Debt Relief Initiative (MDRI) \\ MDRI-eligible debt (SDR million) ${ }^{5} \quad 9.42$ \\ Financed by: MDRI Trust $\quad 7.44$ \\ Remaining HIPC resources $\quad 1.98$}

Debt Relief by Facility (SDR million)

Delivery Date

December 2007

\begin{tabular}{rrr}
\multicolumn{3}{c}{ Eligible Debt } \\
\hline GRA & $\underline{\text { PRGF }}$ & $\underline{\text { Total }}$ \\
N/A & 9.42 & 9.42
\end{tabular}

\footnotetext{
${ }^{2}$ The Fund approved the decision on 12/15/2000 as Decision 12365-(00/126). The World Bank Board decision was taken on 12/14/2000.

${ }^{3}$ Assistance committed under the enhanced HIPC Initiative is expressed in net present value (NPV) terms at the decision point.

${ }^{4}$ Under the enhanced HIPC Initiative, an additional disbursement is made at the completion point corresponding to interest income earned on the amount committed at the decision point but not disbursed during the interim period.

${ }^{5}$ The MDRI provides 100 percent debt relief to eligible member countries that qualified for the assistance. Grant assistance from the MDRI Trust and HIPC resources provide debt relief to cover the full stock of debt owed to the Fund as of end-2004 that remains outstanding at the time the member qualifies for such debt relief.
} 


\section{Safeguards assessments}

An update Safeguards Assessment of the Central Bank of The Gambia (CBG) was conducted in November 2006. This update assessment was performed against the backdrop of previous safeguards findings, which identified significant vulnerabilities in the CBG's safeguards framework. While the CBG has instituted a series of control reforms since that assessment, a key challenge looking forward is to build on existing progress to strengthen remaining vulnerabilities in the CBG's safeguards framework, including controls over international reserves and procedures for extending credit to the government. The update assessment, completed in February 10, 2007, recommended certain priority actions to be taken, including semi-annual audits of monetary program data, the implementation of segregation of duties in the reserves management function, a phased implementation of International Financial Reporting Standards, and an action plan to ensure that government borrowing from the CBG is brought within the statutory limits. The CBG is progressively implementing these recommendations.

\section{Exchange rate arrangement}

Until January 20, 1986, the Gambian currency, the dalasi, was pegged to the pound sterling at a rate of D5 $=£ 1$. On January 20,1986 , an interbank market for foreign exchange was introduced, and since then the exchange rate has been determined by market forces. Effective June 30, 2002, the exchange rate arrangement of The Gambia was reclassified from independently floating to managed float with no preannounced path. As of end-June 2008, the midpoint exchange rate in the interbank market was D20.651 per U.S. dollar. The Gambia has accepted the obligations of Article VIII, Sections 2(a), 3, and 4 and maintains an exchange system that is free of restrictions on the making of payments and transfers for current international transactions. The Gambia maintains exchange restrictions for security reasons, based on UN Security Council Resolutions, that have been notified to the Fund for approval under the procedures set forth in Executive Board Decision No. 144-(52/51).

The Gambia has been part of the exchange rate mechanism of the West African Monetary Zone (WAMZ) since April 2002.

\section{Last Article IV consultation}

The Executive Board concluded the 2006 Article IV consultation on October 13, 2006.

\section{Technical assistance}

The Fund has been providing The Gambia with extensive technical assistance in macroeconomic, fiscal, and monetary areas, and in improving the compilation of macroeconomic statistics. Specific technical assistance projects are the following:

\section{Fiscal Affairs Department}


Jul. 2008

Aug./Sep. 2007

Sep. 2004-May 2006

Feb./Mar. 2004

Apr. 2003

Mar. 2003-Oct. 2003

Dec. 2002

Oct. 2002

Mar. 2002-Oct. 2003

Nov. 2001-Oct. 2003

Jul. 2001

Aug. 2000-Aug. 2001

Sep. 1999

Jan./Feb. 1996
Peripatetic regional advisor followed up on the work of the Aug./Sep. 2007 FAD mission.

TA mission assessed all areas of public financial management and provided an action plan to secure the actual implementation of reforms initiated in the recent past.

Peripatetic regional advisor assisted the authorities in putting the new organic budget law into effect, strengthening public expenditure management, and improving the reporting of budget execution.

Mission worked jointly with the World Bank on the Assessment and Action Plan (AAP).

TA advisor reviewed reforms in public expenditure management.

Long-term resident budget expert helped the authorities to strengthen budgetary expenditure reporting and control.

TA advisor advised the authorities on drafting an organic budget bill.

TA advisor reviewed reforms in public expenditure management.

Long-term resident budget expert helped the authorities to strengthen budgetary expenditure reporting and control, initially for a year beginning from March 2002. The assignment was later extended until October 2003.

Peripatetic advisor assisted the DoSFEA in revenue administration reforms, including customs, implementing a largetaxpayer unit, and establishing a central revenue authority.

TA mission assessed the authorities' capacity to track povertyrelated spending.

Long-term resident budget expert assisted the authorities in strengthening budgetary expenditure reporting and control.

TA mission assisted the authorities in expenditure management, budget execution issues, cash and debt management, short-term financial planning, fiscal reporting, and information systems.

Joint FAD/United Nations Development Program (UNDP) TA mission helped set up a system for monitoring the financial operations of public enterprises.

\section{Monetary and Capital Markets Department/ Monetary and Financial Systems Department /Monetary and Exchange Affairs Department}


Sept. 2007

Mar./May 2007

Mar./Apr 2007

Jan./Feb. 2007

Jul./Aug. 2006

Jul./Aug. 2006

Apr./May 2006

Apr./May 2006

Nov. 2005

Mar. 2005

Oct. 2004

Jul. 2002

Dec. 2001

May 2001

Apr. 2001

May 2000

Nov. 1999
Mission advised on improving the monetary policy framework and enhancing the effectiveness of monetary, foreign exchange, and debt management operations for the CBG.

Technical expert advised the CBG on banking supervision.

Technical expert advised the CBG in strengthening its capacity in internal auditing.

Technical expert advised the CBG on improving monetary operations.

Technical expert advised the CBG on banking supervision.

Mission reviewed progress made in strengthening the CBG's capacity in monetary operations and liquidity forecasting, foreign exchange operations, and foreign reserves management.

Technical expert advised the CBG on banking supervision.

Technical expert advised the CBG on improving monetary operations.

Technical expert advised the CBG on improving monetary operations.

Follow-up to the October 2004 mission.

Advisory mission made recommendations for improving monetary and foreign exchange operations and for reorganizing the central bank.

TA diagnostic mission focused on financial supervision and the insurance sector; and helped review the Central Bank Act and draft the Financial Institutions and Insurance Act.

TA diagnostic mission focused on strengthening CBG ability to , formulate and implement monetary policy and manage its foreign exchange operations and the financial system.

Short-term expert helped the authorities to design operational, prudential, and policy safeguards (including assessing the adequacy of legislation) for introducing foreign-currencydenominated accounts.

Short-term expert helped the authorities to set up a book-entry system.

Short-term expert helped the authorities to set up a short-term liquidity forecasting system.

Short-term expert helped the authorities design operational, prudential, and policy safeguards (including assessing the 
Aug. 1998

Dec. 1996

Jan./Feb. 1994

\section{Statistics Department}

Jun. 2008

Apr./May 2008

Mar. 2008

Oct./Nov. 2007

Sep. 2007

Aug. 2007

Apr./May 2006

Feb. 2006

Feb. 2005 adequacy of legislation) for introducing foreign-currencydenominated accounts in the banking system.

TA mission helped the CBG draft market-based monetary policy instruments and review its program for strengthening banking supervision.

Technical expert helped the CBG in foreign exchange operations.

TA mission worked on monetary management and bank supervision.

Fourth visit of the U.K. Department of International Development (DfID)-funded TA mission helped the authorities improve the compilation of national accounts statistics, particularly in rebasing the GDP series and calculation of GDP by expenditure approach, with results from the 2004 Economic Census.

Follow-up of the 2006 TA mission to assist the CBG in improving the compilation of monetary and financial statistics and in preparing the standardized report forms for reporting monetary data to the IMF.

Third visit of the DfID-funded TA mission helped the authorities improve the compilation of national accounts statistics, particularly in rebasing the GDP series and calculation of GDP by expenditure approach, with results from the 2004 Economic Census.

Second visit of the DfID-funded TA mission helped the authorities improve the compilation of national accounts statistics, particularly in rebasing the GDP series with results from the 2004 Economic Census.

The DfID-funded TA mission helped to improve the compilation of balance of payments statistics.

The DfID-funded TA mission advised in improving the compilation of national accounts statistics, particularly in rebasing the GDP series with results from the 2004 Economic Census.

TA mission helped to improve the compilation and analytical soundness of monetary and financial statistics.

TA mission advised on compilation of balance of payments statistics.

Report on the Observance of Standards and Codes (ROSC) - Data Module - mission assessed data quality in four main areas of macroeconomic statistics (national accounts, government finance, 
$2002-04$

May 2003

Aug. 2001

Sep. 2000

Nov. 1999

Jun./Jul. 1999

Nov./Dec. 1998

\section{Others}

Mar./Apr. 2007

Apr. 2002-Apr. 2004 monetary, and balance of payments) based on the Fund's Data Quality Assessment Framework (DQAF, July 2003) and The Gambia's dissemination practices against the recommendations of the General Data Dissemination System (GDDS).

Peripatetic statistical advisor helped the Central Statistics Department update CPI data and improve the compilation of national accounts statistics.

Mission advised on building an integrated database to report monetary statistics for all IMF data submissions.

TA mission advised on compilation of monetary and financial statistics.

TA mission advised on compilation of balance of payments statistics.

Mission reviewed collection of statistics to develop GDDS metadata for The Gambia.

TA mission advised on compilation of balance of payments statistics.

TA mission advised on national accounts statistics.

A Poverty and Social Impact Analysis (PSIA) mission analyzed the planned reform of the groundnut sector and discussed with the authorities the implications of these reforms.

A long-term resident macroeconomic advisor was assigned initially for a year beginning from April 2002, and later the assignment was extended by one more year through April 2004.

\section{Resident Representative}

Mr. Alex Segura-Ubiergo was appointed the Fund's first Resident Representative to The Gambia in September 2006. He is also the Resident Representative to Senegal. 
The Gambia: Joint Bank-Fund Work Program, August 2008-July 2009

\begin{tabular}{|c|c|c|c|}
\hline Title & Products & Timing of Missions & Expected Delivery Date \\
\hline \multicolumn{4}{|c|}{ A. Mutual Information on Relevant Work Program } \\
\hline $\begin{array}{l}\text { Bank work } \\
\text { program in next } \\
12 \text { months }\end{array}$ & $\begin{array}{l}\text { Economic Management and } \\
\text { Public Service Delivery } \\
\text { 1. Capacity Building for Economic } \\
\text { Management project (including } \\
\text { Integrated Financial Management } \\
\text { Information System) } \\
\text { 2. Development Policy Operation } \\
\text { (budget support) } \\
\text { 3. Poverty Assessment (including } \\
\text { impact of food/oil price increases) } \\
\text { 4. Country Financial } \\
\text { Accountability Assessment } \\
\text { (CFAA) } \\
\text { Growth and Competitiveness } \\
\text { 1. Investment Climate Assessment }\end{array}$ & $\begin{array}{l}\text { 1. Oct./Nov. } 2008 \\
\text { 2. Oct./Nov. } 2008 \\
\text { 3. Oct./Nov. } 2008\end{array}$ & $\begin{array}{l}\text { 1. Project closes end- } \\
\text { 2008 } \\
\text { 2. Target Board date - } \\
\text { Dec. } 2008 / \text { Jan. } 2009 \\
\text { 3. Draft report - Feb. } \\
\text { 2009 } \\
\text { 4. Final report - Nov. } \\
\text { 2008 } \\
\text { 1. Final report - Nov. } \\
\text { 2008 }\end{array}$ \\
\hline $\begin{array}{l}\text { Fund work } \\
\text { program in next } \\
12 \text { months }\end{array}$ & $\begin{array}{l}\text { Policy Advice } \\
\text { 1. Article IV consultation, } 3^{\text {rd }} \\
\text { PRGF review } \\
\text { 2. Fourth PRGF review } \\
\text { 3. Fifth PRGF review } \\
\text { Technical Assistance } \\
\text { 1. Public financial management } \\
\text { (regional PFM Adviser) } \\
\text { 2. Public financial management } \\
\text { (expert visits) } \\
\text { 3. Balance of payments statistics } \\
\text { 4. National accounts statistics }\end{array}$ & $\begin{array}{l}\text { 1. May } 2008 \\
\text { 2. Oct./Nov. } 2008 \\
\text { 3. May } 2009 \\
\text { 1. Oct. } 2008 \text { and Dec. } \\
\text { 2008 } \\
\text { 2. Nov. } 2008 \text { and Mar. } \\
\text { 2009 } \\
\text { 3. Oct. } 2008 \\
\text { 4. Sep./Oct. } 2008\end{array}$ & $\begin{array}{l}\text { 1. Sep. } 8,2008 \\
\text { 2. Jan. } 2009 \\
\text { 3. Jul. } 2009 \\
\text { 1. Oct. } 2008 \text { and Dec. } \\
\text { 2008 } \\
\text { 2. Nov. } 2008 \text { and Mar. } \\
\text { 2009 } \\
\text { 3. Oct. } 2008 \\
\text { 4. Oct. } 2008\end{array}$ \\
\hline \multicolumn{4}{|c|}{$\begin{array}{r}\text { B. Requests for Work Program Inputs } \\
\end{array}$} \\
\hline $\begin{array}{l}\text { Fund request to } \\
\text { Bank }\end{array}$ & $\begin{array}{l}\text { Periodic updates on DPO, poverty } \\
\text { assessment, CFAA, and ICA }\end{array}$ & & \\
\hline $\begin{array}{l}\text { Bank request to } \\
\text { Fund }\end{array}$ & $\begin{array}{l}\text { Periodic updates on } \\
\text { macroeconomic framework }\end{array}$ & & \\
\hline \multicolumn{4}{|c|}{ C. Agreement on Joint Products and Missions } \\
\hline $\begin{array}{l}\text { Joint products } \\
\text { in next } 12 \\
\text { months }\end{array}$ & $\begin{array}{l}\text { 1. Debt Sustainability Analysis } \\
\text { 2. JSAN (First APR on PRSP II) }\end{array}$ & 1. Oct./Nov. 2008 & $\begin{array}{l}\text { 1. Jan. } 2009 \\
\text { 2. Jan. } 2009 \\
\end{array}$ \\
\hline
\end{tabular}




\section{The Gambia: Relations with the African Development Bank}

(As of June 20, 2008)

The African Development Bank (AfDB) Group began lending to The Gambia in 1974. As of March 31, 2008, it had approved 54 operations with total commitments (net of cancellations) of UA205.8 million (US\$338.4 million) in the following sectors: transport (25 percent); agriculture (21.7 percent); social (24.6 percent); public utilities (12.1 percent); multisectoral (8.8 percent); environment ( 5.8 percent); and industry ( 2.0 percent). ${ }^{16}$ About 84.8 percent of the Bank Group's net commitments were made from the resources of the African Development Fund (ADF), 8.6 percent from the AfDB nonconcessional window, and 6.6 percent from the Nigeria Trust Fund (NTF).

As of June 20, 2008, 41 operations had been completed, 2 were cancelled at the government's request, and 11 others continue, including 3 multinational projects, all in agriculture. Implementation of the portfolio is generally satisfactory; it achieved a rating score of 2.01 (on a scale from 0 to 3) during the Bank Group's 2006 portfolio review. The portfolio has a relatively low project-at-risk (PAR) rate of 33.3 percent, which compares well with the Bank-wide average of 43 percent as indicated in the AfDB's 2004 Annual Portfolio Performance Review (APPR). Total disbursement rates for the portfolio are also satisfactory. By June 20, 2008, they were 89.1 percent (overall), 100 percent (AfDB-financed projects), 89.3 percent (ADF), and 72 percent (NTF). The disbursement rate for the ongoing portfolio was 53 percent as of June 20,2008. The satisfactory performance of the portfolio is attributed to more frequent supervision missions conducted by the AfDB, especially since 2003.

The AfDB is also a major participant in The Gambia's enhanced Heavily Indebted Poor Countries (HIPC) Initiative program, under which it is due to grant debt relief of US\$15.8 million in net present value (NPV) terms (23.6 percent of total debt relief under the program). Of this, $\$ 6.3$ million in end-1999 NPV has been paid as interim relief. Additionally, MDRI relief from the AfDB will yield annual debt service savings (net of HIPC assistance) averaging US $\$ 1.6$ million over the next 5 years and US $\$ 3.9$ million over the following 39 years.

The AfDB has recently prepared a Joint Assistance Strategy (JAS) with the World Bank to cover 2008-11. The JAS is based on two main pillars - (1) strengthening the institutional framework for economic management and public service delivery, and (2) enhancing growth and competitiveness and the productive capacity of the poor. The JAS will help support the Government's national priorities through the main channels of budget and project support in the context of growth poles and enhanced service delivery. The strategy was approved at the

\footnotetext{
${ }^{6} \mathrm{UA}$ stands for unit of account $=1$ SDR (equivalent to about $\$ 1.6445$ as of March 31, 2008).
} 
World Bank in February 2008 and at the AfDB in March 2008. The JAS is anchored to the Gambia's Poverty Reduction Strategy Paper PRSP II (2007-2011) and will be supported by new lending and by the ongoing portfolio, which is summarized in Table 2. Table 1 below describes some of the recent projects in the portfolio:

Table 1. Some Recent Projects in the Portfolio

\begin{tabular}{|c|c|c|}
\hline Objective & $\begin{array}{l}\text { Instrument } \\
\text { and Amount }\end{array}$ & Focus \\
\hline Meet the MDGs & $\begin{array}{l}\text { Basic Education } \\
\text { Project, } \\
\text { UA10.0 million }\end{array}$ & $\begin{array}{l}\text { Increase access to quality education and } \\
\text { skills development, particularly for girls } \\
\text { and pupils in the poorest areas of the } \\
\text { country. }\end{array}$ \\
\hline Reduce poverty & $\begin{array}{l}\text { Entrepreneurship } \\
\text { development and } \\
\text { livelihood promotion } \\
\text { UA8.0 million }\end{array}$ & $\begin{array}{l}\text { Enhance entrepreneurial skills and improve } \\
\text { capacity and income generation ability by } \\
\text { providing microfinance. Focus is poverty } \\
\text { reduction. }\end{array}$ \\
\hline $\begin{array}{l}\text { Implement multi- } \\
\text { sector capacity } \\
\text { building (planned } \\
\text { for early } 2008 \text {; } \\
\text { articulated in the } \\
2006 \text { CSP update) }\end{array}$ & $\begin{array}{l}\text { Institutional Support } \\
\text { Project for Economic } \\
\text { Management and } \\
\text { Governance, grant of } \\
\text { UA1.4 million }\end{array}$ & $\begin{array}{l}\text { Strengthen capacity of departments and } \\
\text { institutions involved in preparing and } \\
\text { implementing the PRSP/SPA-II. Extend } \\
\text { support to institutions dealing in economic } \\
\text { governance, including the new Directorate } \\
\text { of Central Project Management and Aid } \\
\text { Coordination in the Department of State } \\
\text { for Finance and Economic Affairs } \\
\text { (DOSFEA), National Audit Office (NAO), } \\
\text { and SPACO. }\end{array}$ \\
\hline
\end{tabular}

Under ADF-10 and ADF-11, The Gambia is a grants-only recipient. Additional resources can be made available if there is improvement in both performance under the Country Policy Institutional Assessment (CPIA) and portfolio performance.

The AfDB's strategy is implemented through both lending and nonlending activities. Lending activities will comprise project finance and budget support. Nonlending intervention is designed to strengthen policy dialogue between the government and stakeholders and focuses mainly on studies, funded through grants, to improve governance, mainstream gender, enhance efficiency of infrastructure, and improve the energy supply. The AfDB prepared a Governance Profile for The Gambia in 2007 and has recently collaborated with the World Bank and the U.K. Department for International Development (DfID) in the preparation of a study on Civil Service Reform. 
Table 2. Ongoing Portfolio as of June 20, 2008

\begin{tabular}{|c|c|c|c|c|c|c|c|}
\hline Sector/Project & $\begin{array}{l}\text { Approval } \\
\text { Date }\end{array}$ & $\begin{array}{l}\text { Completion } \\
\text { Date }\end{array}$ & Remarks & $\begin{array}{c}\text { Financing } \\
\text { Source }\end{array}$ & $\begin{array}{l}\text { Amount Approved } \\
\text { (UA million) }\end{array}$ & $\begin{array}{l}\text { Disbursements } \\
\text { (UA million) }\end{array}$ & $\%$ \\
\hline $\begin{array}{l}\text { Agriculture } \\
\text { 1. Participatory. Integrated Watershed Management. }\end{array}$ & $09 / 06 / 04$ & $31 / 12 / 11$ & & NTF Loan & 4.95 & 2.50 & 50.5 \\
\hline 2. Artisanal Fisheries Development & $17 / 05 / 00$ & $30 / 06 / 09$ & & NTF Loan & 2.90 & 1.68 & 58 \\
\hline 3. NERICA Rice Dissemination- Gambia (multinational) & $26 / 09 / 03$ & $31 / 12 / 10$ & & ADF Loan & 1.56 & 0.31 & 20 \\
\hline 4. Farmer Managed Rice Irrigation Project & $26 / 04 / 05$ & $30 / 04 / 11$ & & $\begin{array}{l}\text { ADF Loan } \\
\text { ADF Grant }\end{array}$ & $\begin{array}{l}5.00 \\
0.50\end{array}$ & $\begin{array}{r}1.1 \\
0.132\end{array}$ & $\begin{array}{l}21.8 \\
26.4\end{array}$ \\
\hline 5. Invasive Aquatic Weeds-Gambia (multinational) & $22 / 09 / 04$ & $31 / 12 / 11$ & & ADF Loan & 0.31 & 0.092 & 29.6 \\
\hline $\begin{array}{c}\text { Total Agriculture } \\
\end{array}$ & & & & & 15.22 & 5.81 & 38.2 \\
\hline $\begin{array}{l}\text { Social Sector } \\
\text { 6. Basic Education III }\end{array}$ & $11 / 09 / 02$ & $30 / 06 / 08$ & & ADF Loan & 10.00 & 8.41 & 84.1 \\
\hline 7. Health Services Development Project & $10 / 12 / 97$ & $31 / 12 / 08$ & & ADF Loan & 7.00 & 6.37 & 91.1 \\
\hline 8. Community Skills Development Project & $16 / 02 / 00$ & $31 / 12 / 08$ & & $\begin{array}{l}\text { ADF Loan } \\
\text { ADF Grant }\end{array}$ & $\begin{array}{l}4.44 \\
1.45 \\
\end{array}$ & $\begin{array}{l}3.05 \\
0.86\end{array}$ & $\begin{array}{l}68.8 \\
59.2\end{array}$ \\
\hline $\begin{array}{l}\text { 9. Entrepreneurship Promotion and } \\
\text { Microfinance Development Project }\end{array}$ & $15 / 11 / 06$ & $31 / 12 / 12$ & & ADF Grant & 8.0 & 0.67 & 8.4 \\
\hline $\begin{array}{r}\text { Total Social Sector } \\
\end{array}$ & & & & & 30.89 & 19.36 & 62.7 \\
\hline $\begin{array}{l}\text { Multi-sector } \\
\text { 10. Institutional Support Project for Economic and Financial } \\
\text { Governance }\end{array}$ & $21 / 11 / 07$ & $31 / 12 / 10$ & & ADF Grant & 1.4 & 0.0 & 0.0 \\
\hline $\begin{array}{c}\text { Total Multi-sector } \\
\end{array}$ & & & & & 1.4 & $\mathbf{0 . 0}$ & $\mathbf{0 . 0}$ \\
\hline Total Ongoing Portfolio & & & & & 47.51 & 25.17 & 53 \\
\hline
\end{tabular}

\begin{tabular}{|l|r|r|}
\hline \multicolumn{1}{|c|}{ Sector } & \multicolumn{1}{c|}{$\begin{array}{c}\text { Net Amount } \\
\text { Approved (UA } \\
\text { Million) }\end{array}$} & $\begin{array}{c}\text { Percentage Share } \\
\text { (\%) }\end{array}$ \\
\hline Agriculture & 15.22 & 32.0 \\
\hline Social Sector & 30.89 & 65.0 \\
\hline Multi-sector & 1.40 & 3.0 \\
\hline Total & $\mathbf{4 7 . 5 1}$ & $\mathbf{1 0 0 \%}$ \\
\hline
\end{tabular}




\section{The Gambia: Statistical Issues}

Data provision has some shortcomings, but is broadly adequate for surveillance. While the authorities have made some progress in improving the compilation of economic and financial statistics, substantial shortcomings remain in national accounts, balance of payments, and external debt statistics. Data reporting to the Fund is somewhat irregular. The country participates in the General Data Dissemination System (GDDS), with its metadata last updated in the second half of 2003.

The National Assembly passed a new Statistics Act in December 2005 and work began in June 2006 to implement the plan for transforming the Central Statistics Department (CSD) into The Gambia Bureau of Statistics (GBoS). A Statistics Council and a Statistician General have already been appointed and efforts are ongoing to fully staff the GBoS. A mission visited Banjul in February 2005 to prepare a data ROSC, which was published by the Fund in November 2005.

\section{Real sector}

The main constraints to improving national accounts include inadequate source data due to low response rates of surveys (manufacturing, trade, and business services industries), as well as poor quality of external data and inattention to other important sources (such as the household budget survey, livestock census, and census of industrial production). The GBoS continues to face human and financial constraints to undertake such surveys and process the data.

STA missions on national accounts in 2007 and March 2008 assisted the GBoS to implement the 1993 System of National Accounts methodology and rebase the national accounts to properly reflect the country's output levels, economic structure and relative prices. In this context, STA missions have helped the authorities: (1) process the data collected for the 2004 Economic Census; (2) rebase the GDP series using the results of the 2004 Economic Census; and (3) improve the GDP estimates by the production approach and begin compiling GDP series by the expenditure approach. The authorities now have a preliminary revised nominal GDP series compiled by the production approach for the period 2000-07 (with a base year of 2004). GDP series by the expenditure approach are still under review. In May 2007, the country began participating in the second phase of the GDDG Project for Anglophone Africa on national accounts, which is funded by the U.K. Department for International Development (DfID).

The World Bank has been providing technical assistance to the GBoS to update the consumer price index (CPI) using the 2003 household expenditure survey to better reflect current consumption patterns. The GBoS began to publish in early 2007 a new national CPI with representative expenditure basket as of August 2004. 


\section{Government finance}

The authorities release data on central government transactions with a lag of about four weeks for both revenue and expenditure. The central government accounts for 1991-99 were not audited until 2005. Inadequacies persist in compiling data on an economic basis and in tracking foreign-financed expenditure. Monthly data on domestic government financing are available with a delay of six to eight weeks. At a meeting with STA in October 2007, the authorities expressed interest in technical assistance to facilitate the migration to GFSM2001. No data are being reported for publication in the Government Finance Statistics Yearbook or in the IFS.

\section{Monetary data}

The Central Bank of The Gambia (CBG) has improved data reporting to the Fund, but sometimes the reports are delayed. An April-May 2006 STA mission recommended: expanding the coverage of depository corporations to include credit unions; including accrued interest in the value of financial instruments; and compiling a financial survey given the significant influence of the Social Security and Housing Finance Corporation in monetary developments. To improve the accuracy and classification of government accounts, the mission designed a supplementary form for reporting government positions at the CBG, to be reported to the IMF monthly. It also assisted the $\mathrm{CBG}$ in the introduction of standardized report forms (SRFs). A follow-up mission in April/May 2008 made further progress on the SRFs for reporting data on the central bank and other depository corporations to STA and establishing an integrated monetary database, which would generate alternative outputs for use by the CBG, STA, and AFR. The authorities have now prepared a preliminary set of SRFs.

\section{External sector statistics}

Despite recent improvements, balance of payments statistics continue to be affected by shortcomings. These include long delays in the collection of trade, customs, and tourist arrival data; crude methods of estimating re-export trade; poor data on capital flows; lack of a register of firms and establishments engaged in external transactions; poor classification of balance of payments data; and lack of consistent methodology. Institutional weaknesses have also been a major impediment to improving statistics. Official grant and loan disbursements and repayments are generally well recorded, but there are some gaps in project disbursements.

The CBG produces balance of payments statistics according to the Balance of Payments Manual, $5^{\text {th }}$ edition (BPM5). These statistics are published in International Financial Statistics (IFS) and in the 2007 IMF Balance of Payments Statistics Yearbook (BOPSY 2007). The CBG has been compiling quarterly balance of payments statistics through a Fund- 
administered technical assistance project funded by DfID. The most recent data available are for the first quarter of 2008.

With DfID assistance the CBG conducted an enterprise survey in March 2006 to collect data for the international investment position. In April 2006, the CBG also initiated a survey funded by the World Bank to collect data on selected components of the current account. 
The Gambia: Table of Common Indicators Required for Surveillance

(As of July 21, 2008)

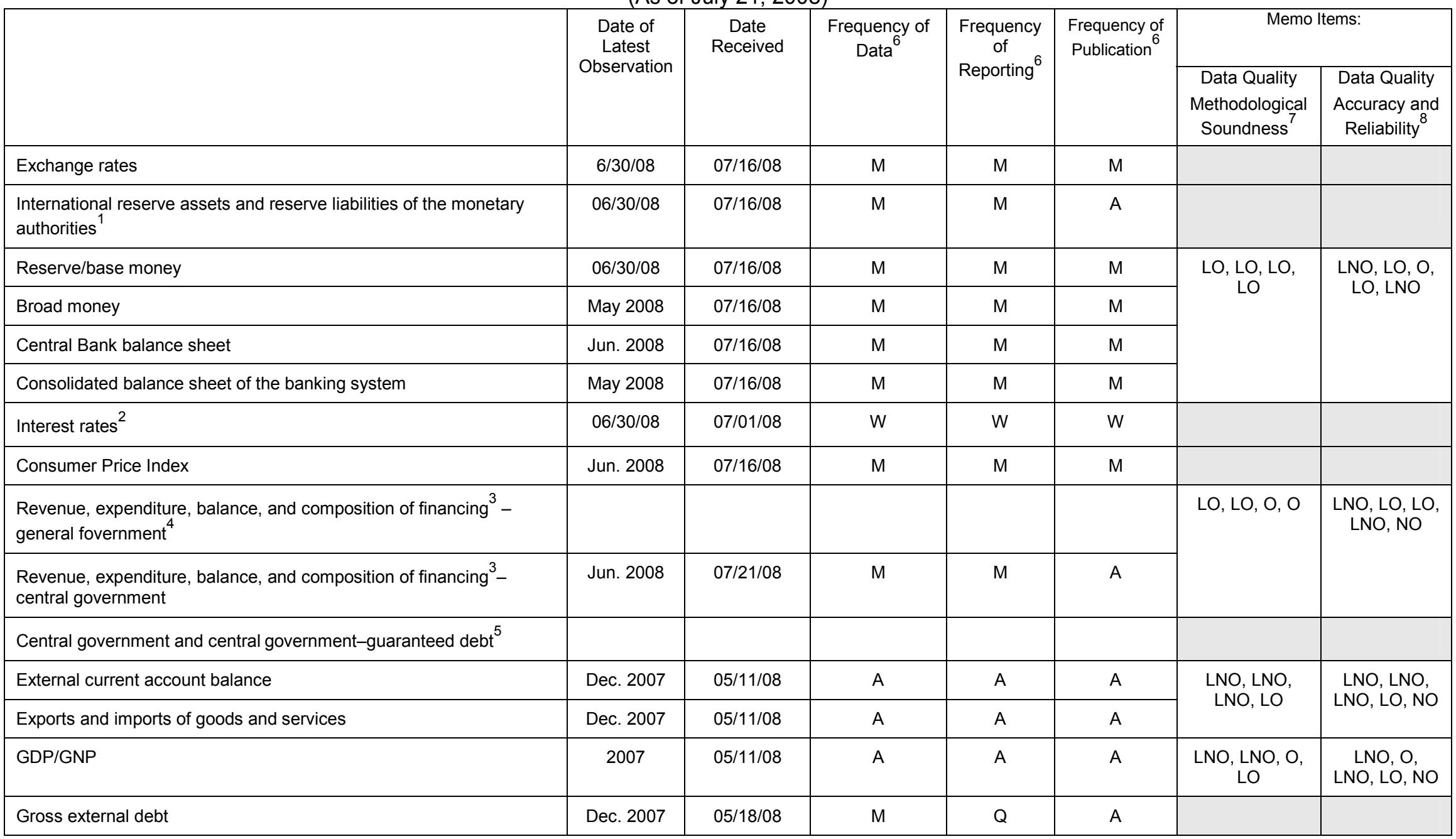

${ }^{1}$ Includes reserve assets pledged or otherwise encumbered as well as net derivative positions.

${ }^{2}$ Both market-based and officially determined, including discount rates, money market rates, and rates on treasury bills, notes, and bonds.

${ }^{3}$ Foreign, domestic bank, and domestic nonbank financing.

The general government consists of the central government (budgetary funds, extrabudgetary funds, and social security funds) and state and local governments.

${ }^{5}$ Including currency and maturity composition.

${ }^{6}$ Daily $(D)$, weekly $(W)$, monthly (M), quarterly (Q), annually (A); irregular (I); not available (NA)

${ }^{7}$ Reflects the assessment provided in the data ROSC published on November 8, 2005, and based on the findings of the mission in February 2005. For the dataset corresponding to the variable in each row, the assessment indicates whether international standards concerning (respectively) concepts and definitions, scope, classification/sectorization, and basis for recording are fully observed ( $\mathrm{O})$, largely observed

(LO), largely not observed (LNO), or not observed (NO).

Same as footnote 7, except referring to international standards concerning (respectively) source data, assessment and validation of source data, statistical techniques, assessment and validation of intermediate data and statistical outputs, and revision studies. 
Public Information Notice (PIN) No. 08/121 FOR IMMEDIATE RELEASE

September 22, 2008
International Monetary Fund

$70019^{\text {th }}$ Street, NW

Washington, D. C. 20431 USA

\section{IMF Executive Board Concludes 2008 Article IV Consultation with The Gambia}

On September 8, 2008, the Executive Board of the International Monetary Fund (IMF) concluded the Article IV consultation with The Gambia. ${ }^{1}$

\section{Background}

Over the last two years, The Gambia has maintained macroeconomic stability, sustained high growth and made progress toward achieving debt sustainability. Fiscal performance improved and monetary policy remained geared to maintaining low inflation. Real GDP growth, which has averaged $61 / 2$ percent a year since 2004 , has been led by the construction, tourism and telecommunications sectors, facilitated by a steady inflow of foreign direct investment. However, performance of the agriculture sector-critical for achieving the government's poverty reducing objectives-has been mixed. In particular, groundnut output fell substantially in the 2007/08 season largely due to a poor pattern of rainfall.

Inflation has abated after a spike in 2007. Disruptions in the supply of foodstuffs from neighboring countries and rising import costs pushed the annual rate of inflation from less than 1 percent in December 2006 to 6-7 percent during most of 2007. In the first half of 2008, tight monetary policy and the lagged effect of a sharp appreciation of the dalasi in the third quarter of 2007 helped contain inflationary pressures from increases in world food and fuel prices. The annual rate of inflation fell to 2 percent in June 2008.

1. ${ }^{1}$ Under Article IV of the IMF's Articles of Agreement, the IMF holds bilateral discussions with members, usually every year. A staff team visits the country, collects economic and financial information, and discusses with officials the country's economic developments and policies. On return to headquarters, the staff prepares a report, which forms the basis for discussion by the Executive Board. At the conclusion of the discussion, the Managing Director, as Chairman of the Board, summarizes the views of Executive Directors, and this summary is transmitted to the country's authorities. 
The central government budget improved markedly in 2007 , going from an overall deficit of 7.8 percent of GDP in 2006 to a surplus of 0.5 percent of GDP in 2007. Total expenditure and net lending fell sharply, reflecting substantially lower externally financed capital expenditures than budgeted for 2007 . The stock of public domestic debt fell from 32 percent to 28 percent of GDP.

The Gambia's external current account deficit widened from $11 \frac{1}{2}$ percent of GDP in 2006 to $12 \frac{1}{2}$ percent in 2007 . While the trade balance remained unchanged, an improvement in the services balance was more than offset by a decline in remittances and official transfers. The deficits were financed largely by inflows of foreign direct investment.

The Gambia's external debt position was helped by the delivery of HIPC and MDRI debt relief at the end of 2007, resulting in the stock of external debt falling from 110 percent of GDP to 50 percent. However, The Gambia remains at high risk of debt distress because of the high level of outstanding debt and the country's vulnerability to shocks.

The Gambia's macroeconomic policies are being supported by a three year PRGF arrangement approved in February 2007. The program's objectives draw on the strategic priorities set out in The Gambia's second Poverty Reduction Strategy Paper which include: (i) macroeconomic stability and effective management of public resources; (ii) pro-poor growth and employment through development of the private sector; and (iii) improved provision of basic services.

\section{Executive Board Assessment}

Executive Directors commended the Gambian authorities for their prudent fiscal and monetary policies, which have laid a solid foundation for macroeconomic stability. Despite the increases in world food and fuel prices, growth has remained strong, per capita GDP has increased significantly, and inflation has been contained at low singledigit levels.

Directors agreed that The Gambia's major economic challenge will be to sustain strong growth and continue to reduce poverty, which remains widespread. They encouraged the authorities to continue prudent macroeconomic policies while boosting povertyreducing government expenditures, in line with the targets in the country's PRSP, to make faster progress towards achieving the Millennium Development Goals.

Directors welcomed the authorities' measured response to the recent adverse international price shocks. The sales tax on rice-the principal staple food in the country-was eliminated to mitigate the impact of its rising cost, while compensating revenue-raising measures were implemented to safeguard the fiscal position. Directors commended the authorities for adjusting retail petroleum product prices to avoid budget subsidization. They recommended that any further support for the most vulnerable households, if needed, be provided through carefully-targeted programs rather than through general subsidies. 
Directors acknowledged the authorities' solid revenue effort, underpinned by improved tax administration. They encouraged the authorities to undertake further reforms to broaden the tax base and make the tax system more buoyant. They suggested that the authorities review import duty exemptions with a view to reducing their scope. Further progress in strengthening public financial management will be desirable in order to ensure that budget formulation is realistic and in line with PRSP priorities.

Directors commended the Central Bank of The Gambia (CBG) for keeping inflation in check under challenging circumstances. In view of the uncertainties about the monetary policy transmission mechanism, most Directors encouraged the CBG to supplement it money targeting framework with a range of indicators, and to use foreign exchange operations as an additional instrument to influence domestic liquidity conditions.

Directors agreed that The Gambia's flexible exchange rate system has served the country well, with market interventions limited to preventing disorderly adjustment and replenishing foreign reserves, as needed. They took note of the staff's assessment that the real exchange rate of the dalasi appears to be in line with fundamentals. They encouraged the authorities to take steps to enhance the country's investment climate and international competitiveness, by addressing the heavy burden of central and local government taxation on business, and by investing in infrastructure, education, and health.

Directors considered that The Gambia's banking sector is fundamentally sound, while noting the substantial scope to further deepen financial intermediation. They welcomed the authorities' efforts to strengthen the prudential supervision of banks. They looked forward to the functioning of the national credit reference bureau, which should bolster the prudent extension of credit and support private sector development and growth.

Directors noted that HIPC and MDRI debt relief provided at end-2007 has improved significantly The Gambia's external debt indicators. Nonetheless, The Gambia remains at high risk of debt distress due to the high level of outstanding debt and the country's vulnerability to shocks. Directors urged the authorities to speed up the formulation of a national debt strategy and to rely primarily on grants to finance their development plans.

Public Information Notices (PINs) form part of the IMF's efforts to promote transparency of the IMF's views and analysis of economic developments and policies. With the consent of the country (or countries) concerned, PINs are issued after Executive Board discussions of Article IV consultations with member countries, of its surveillance of developments at the regional level, of post-program monitoring, and of ex post assessments of member countries with longer-term program engagements. PINs are also issued after Executive Board discussions of general policy matters, unless otherwise decided by the Executive Board in a particular case. 
The Gambia: Selected Economic Indicators, 2004-07

$2004 \quad 2005 \quad 2006 \quad 2007$

Est.

National income and prices (percentage change)

$\begin{array}{lrrrr}\text { GDP at constant prices } & 7.0 & 5.1 & 6.5 & 6.3 \\ \text { Inflation (period average) } & 14.3 & 5.0 & 2.1 & 5.4\end{array}$

External sector

Current account balance incl. official transfers (percent of GDP)

$\begin{array}{rrrr}-6.1 & -15.1 & -11.5 & -12.5 \\ 10.5 & -16.5 & 3.9 & 8.8 \\ 46.2 & 10.2 & -0.6 & 18.4 \\ -1.2 & 6.3 & -0.3 & 10.7 \\ 84.0 & 96.6 & 118.6 & 141.5 \\ 4.3 & 4.5 & 5.5 & 5.5\end{array}$

Money and credit (in percent change of beginning of the year broad money)

Broad money

Credit to the private sector

Yield on 91-day treasury bill (percent per year)

Central government budget (percent of GDP)

Domestic revenues

Grants

Total expenditure and net lending

Overall balance

Basic balance ${ }^{1}$

Nominal stock of public debt (\% of GDP)

Domestic

External $^{2}$

$\begin{array}{rrrr}18.3 & 13.1 & 26.2 & 6.7 \\ -6.5 & 5.5 & 8.4 & 4.3 \\ 28.0 & 12.5 & 10.4 & 11.9\end{array}$

$\begin{array}{llll}20.9 & 19.7 & 21.2 & 21.7\end{array}$

$\begin{array}{llll}4.5 & 1.7 & 1.3 & 1.2\end{array}$

$\begin{array}{llll}31.1 & 30.0 & 29.6 & 22.7\end{array}$

$\begin{array}{llll}-6.2 & -9.2 & -7.8 & 0.5\end{array}$

$\begin{array}{llll}2.4 & -0.1 & 1.4 & 3.8\end{array}$

\footnotetext{
${ }^{1}$ Domestic revenues minus expenditure and net lending, excluding externally financed capital expenditure.

${ }^{2}$ Reflects HIPC and MDRI debt relief delivered at end-2007.
} 
Press Release No. 08/203

FOR IMMEDIATE RELEASE

September 8, 2008
International Monetary Fund

Washington, D.C. 20431 USA

\section{IMF Executive Board Completes Third Review under PRGF Arrangement for The Gambia and Approves US\$3.1 Million Disbursement}

The Executive Board of the International Monetary Fund (IMF) has completed the third review of The Gambia's performance under a program supported by a three-year Poverty Reduction and Growth Facility (PRGF) arrangement. The completion of the review allows for the disbursement of SDR 2 million (about US\$3.1 million), which would bring total disbursements under the arrangement to SDR 8 million (about US\$12.4 million).

The Executive Board also approved the authorities' request for waivers of nonobservance of four structural performance criteria concerning the submission of two special audit reports, the making operational of a credit reference bureau, and the establishment of a central register of fiscal expenditure commitments.

The PRGF arrangement with The Gambia was approved on February 21, 2007 (see Press Release No. 07/28) for an amount of SDR 14 million (about US\$21.7 million).

Following the Executive Board's discussion, Mr. Takatoshi Kato, Deputy Managing Director and Acting Chair, said:

"The Gambian authorities are to be commended for maintaining macroeconomic stability in the context of robust growth and moderate inflation. The PRGF-supported economic program has been implemented satisfactorily, despite the recent increases in world food and fuel prices.

"Over the medium term, The Gambia will face the challenge of maintaining strong growth while further reducing poverty and attaining the Millennium Development Goals. To this end, the authorities are committed to promoting fiscal discipline, private sector growth, and investment in infrastructure, education, and health. The authorities are advised to consider steps to improve The Gambia's international competitiveness through measures to improve the business climate, such as alleviating the burden of central and local government taxation on businesses.

"The Gambia has made strong and successful efforts to improve revenues, in particular through improvements in tax administration. The authorities recognize the need to focus now on broadening the tax base and making it more buoyant, including by reducing the scope of 
import duty exemptions. Making further progress in strengthening public financial management will also help to ensure that budget formulation is realistic and in line with priorities in the country's Poverty Reduction Strategy Paper (PRSP).

"The current stance of monetary policy appears broadly appropriate, and has been able to contain inflationary pressures so far. Exchange rate appreciation has also helped to restrain inflation. The authorities should continue to pass through the increases in international food and fuel prices to consumers, while considering carefully-targeted measures to help the poor.

"Despite receiving debt relief under the Heavily Indebted Poor Countries (HIPC) Initiative and under the Multilateral Debt Relief Initiative (MDRI) at end-2007, The Gambia remains at high risk of debt distress due to the high level of outstanding debt and the country's vulnerability to external shocks. The authorities are formulating a national debt strategy to guide future borrowing decisions, but intend to rely primarily on grants to finance their development program," Mr. Kato said.

The PRGF is the IMF's concessional facility for low-income countries. PRGF loans carry an annual interest rate of 0.5 percent and are repayable over 10 years with a $5 \frac{1}{2}$-year grace period on principal payments. 


\section{Statement by Peter Gakunu, Executive Director for The Gambia and Leonia Lephoto, Advisor to the Executive Director September 8, 2008}

\section{Introduction}

Our Gambian authorities are grateful for the constructive engagement by staff during the consultations. They thank the Fund Executive Board and management for their continued support. The authorities are in broad agreement with the thrust of the staff reports. The Gambia has made significant progress towards macroeconomic stability in recent years and performance under the program has continued to be strong. All the quantitative performance criteria for the period ending March 2008 were met. Four of the eight structural performance criteria were comfortably met while two were implemented with a slight delay. The remaining criteria, related to the full operationalization of the credit reference bureau and the register of government commitments for externally financed projects were partially met. The authorities have since taken remedial steps, including the completion of the register of externally financed projects in July. With regard to the credit bureau, it had been set up on time but was not able to operate due to a legal obstacle that had not been envisaged. The authorities have done a review of the relevant legislation and have initiated the process of getting the law modified to facilitate the credit bureau's full operationalization. Accordingly, they are requesting waivers for the missed performance criteria.

\section{Recent Economic Developments}

Gambia's macroeconomic performance continued to be strong with real GDP growth estimated to have averaged $6 \frac{1}{2}$ percent a year in 2006-07. Economic growth was led by robust telecommunications, tourism and construction activities. Following a sharp rise driven by high international food and oil prices in 2007, inflation has slowed down in 2008 . The appreciation of the dalasi and the relatively tight monetary policy has helped to contain inflationary pressures from rising world food and oil prices.

Overall fiscal performance has improved significantly, registering an overall surplus in 2007 and in the first quarter of 2008. This reflected strengthening in tax administration, which boosted revenues, and unfortunately a decline in capital expenditures on account of shortfalls in external financing, including the delayed grant disbursements for a large EU-supported roads project. Government domestic debt has continued to fall and was 28 percent of GDP at end-2007, as the government cleared more domestic arrears than programmed and is planning to clear the remaining stock of arrears by the end of 2008.

The sharp appreciation of the dalasi during the third quarter of 2007 was mainly a result of changes in market sentiments. Although the Central Bank of Gambia (CBG) intervention stabilized the situation in the foreign exchange market for the remainder of 2007, the appreciation resumed in 2008 due to strong inflow of remittances and reduced debt service payments. 
Despite the slight widening of the current account deficit as a result of lower transfers, an improvement in the overall external position was realized in 2007. The current account deficit was more than financed by foreign direct investment (FDI) inflows and official concessional loans. As a result, the level of foreign reserves was estimated to have risen to 5.5 months of import coverage by the end of the year.

\section{Performance under the program}

The authorities' dedication to macroeconomic stability continued to ensure successful implementation of the program. All the quantitative program targets for end-March 2008 were met, and the authorities were able to accelerate the elimination of domestic arrears. Four of the eight structural performance criteria were comfortably met while two were implemented with a slight delay.

The remaining criteria, which pertained to the full operationalization of the credit reference bureau and the register of government commitments for externally financed projects were only partially met. The delay in operationalizing the credit reference bureau was caused by the limitations on data sharing under the current financial institutions laws. The authorities have already established the credit reference bureau and banks are providing information to it. The legislation has been reviewed to allow for, among others, the sharing of customer information among the financial institutions and the process of amending it has been started. With regard to the register of government commitments to externally financed projects information needed for the register had been collected. The authorities have filled the gaps with regard to some individual projects, and completed the register by July 15, 2008.

\section{Macroeconomic policies}

The authorities have made significant strides towards the achievement of their goals of consolidating macroeconomic stability and fostering conditions conducive to high and sustainable growth as well as poverty reduction. They remain committed to the implementation of prudent macroeconomic management.

\section{Fiscal policy}

Ensuring fiscal sustainability through continued implementation of prudent fiscal policies remains the authorities' priority. Fiscal policy will aim at containing public debt and related interest payments at sustainable levels, in line with the program.

Revenue performance is thus expected to remain strong in the near to medium term, with continued improvements in tax administration. The authorities are cognizant of the need to review tax policy to broaden the tax base and boost revenues, as efficiency gains from improved revenue administration could be approaching full realization. Such a review will include consideration for streamlining the exemptions on import duties. However, the implementation of the Economic Partnership Agreement (EPA), currently being negotiated between the ECOWAS and the EU, is expected to have adverse effects on revenue, which 
could represent a fiscal shock to The Gambia, depending on the pace of implementation of the EPA.

The main challenge confronting the authorities is how to address the effects of the high international food and oil prices while maintaining macroeconomic stability. So far, appreciation of the dalasi has helped contain the impact of the price increases. After reducing the tax on rice from 15 percent to 5 percent in July 2007, in order to help keep rice affordable, the government eliminated the sales tax on rice imports in May 2008. Rice is an essential commodity in the consumption basket of the poor in The Gambia. With regard to the price of oil, the authorities plan to alleviate the impact on the poor by cross-subsidizing kerosene through petrol and diesel prices.

The authorities will continue to make efforts to enhance the efficiency and transparency of fiscal operations. Significant improvements in commitment controls, budget execution and monitoring of PRSP related spending have been realized since the introduction of IFMIS in January 2007. The recently established register of capital projects will help strengthen the system for allocation and monitoring of government counterpart funds to externally-financed capital projects. Efforts to strengthen the budget formulation process, enhance accountability in the use of public resources and improve the quality of public projects will also be continued.

In order to enhance effectiveness of the public sector, the authorities are undertaking a comprehensive civil service reform program with the support of the World Bank. The reform is expected to enable the authorities to attract and retain highly qualified professional staff. The Gambia's external debt has been significantly reduced by the HIPC and MDRI relief. However, the country continues to be at a high risk of debt distress. Delivery of the promised relief by the Paris Club bilateral creditors would further alleviate the debt problem. The authorities are making efforts to obtain additional debt relief from all non-Paris Club bilateral creditors. The authorities are committed to safeguarding external stability through prudent management of external debt. In that regard, they are planning to prepare a comprehensive debt management strategy. To provide input into the strategy they will undertake an independent debt sustainability assessment (DSA) with the assistance of the Commonwealth Secretariat. The new debt strategy will guide The Gambia's debt policy going forward. In the meantime, the government will continue to observe the indicative limits, established under the PRGF-supported program, on contracting of new debt and will limit new borrowing to highly concessional terms. These limits will be revisited within the context of program reviews, once the government's strategy is developed and government is able to conduct it own DSAs.

\section{Monetary and Financial Sector Policies}

The monetary policy objective of the CBG is to maintain price stability. The money targeting framework is used to conduct monetary policy and the rediscount rate to signal changes in policy stance. Increasing international prices of food and oil are expected to pose a challenge to monetary policy from the second half of 2008 onwards. 
The authorities welcome the staff assessment that the Dalasi is in line with fundamentals. The CBG remains committed to maintaining a flexible exchange rate system. However, it will occasionally intervene in exchange markets to prevent disorderly adjustment, correct any misalignment of the exchange rate, and build up foreign reserves or prevent their depletion.

This will be conducted in line with its well-defined intervention policy.

The authorities intend to pursue measures to enhance the CBG's operational independence and its monetary policy execution capacity. These will include ensuring compliance with the provisions of the CBG Act (2005) on its capital requirements and limits on lending to government.

Coordination of monetary and fiscal policies will remain an important part of the authorities' macroeconomic agenda. This will be led by the memorandum of understanding signed by the Department of State for Finance and Economic Affairs (DoSFEA) in 2007 and the CBG to guide domestic debt management and monetary operations. To enhance the effectiveness of monetary policy, the government will continue to replenish the treasury bill special deposit account to mitigate the constraints to the CBG's monetary operations arising from low balances in the account.

The banking sector in The Gambia is sound. However, private sector lending remains weak as the banks continue to be risk averse. To facilitate financial intermediation, the CBG has established a credit reference bureau which will provide the financial sector with information on the credit worthiness of potential borrowers. Banks have started providing data on their customers to the credit bureau. However, the information cannot yet be accessed as the current legislation does not permit sharing information on individual customers. The ongoing review of the financial sector legislation is expected to, among others, remove this restriction and facilitate full operationalization of the credit bureau. The authorities are continuously strengthening prudential supervision of banks. They have made significant headway in the use of the Prompt Corrective Action (PCA) framework, introduced in 2007, to monitor bank compliance and performance against a number of critical indicators. They appreciate the assistance they have thus far received from the Fund in strengthening their banking supervision capacity.

\section{Growth and Poverty Reduction}

The authorities are confronted with the challenge of translating economic growth into the improvement of the economic well-being of the majority of the people, more than 60 percent of whom are estimated to live below the poverty line. The Gambia's second PRSP (PRSP II) attempts to address this through the promotion of macroeconomic stability and effective public resource management, pro-poor growth and employment through private sector development, as well as improvement in the provision of basic services. However, significant funding resources and strengthened capacity in the public service will be needed for the successful implementation of the PRSP II and the achievement of the Millennium Development Goals. The assistance of international development partners will be very important in this regard. 


\section{Conclusion}

We would like to reaffirm the authorities' commitment to prudent macroeconomic policies, growth promotion and improving the livelihood of the people. They appreciate the support they have received from the Fund and the international community thus far. We encourage The Gambia's development partners to continue supporting the country to alleviate the enormous capacity and financial constraints they face. The recently received HIPC and MDRI debt relief has been beneficial in creating some fiscal space which will facilitate allocation of resources to development and poverty reducing activities. Additional debt relief from the remaining creditors would also be very beneficial. 